\title{
Stereoselective Access to Conjugated and Cross- conjugated Dienoates by Rh- and Ru-Catalyzed Isomerizations of Vinylcyclopropanes
}

\author{
Michele Garbo and Clément Mazet* \\ † Department of Organic Chemistry, University of Geneva, \\ 30 quai Ernest Ansermet, 1211 Geneva, Switzerland. \\ clement.mazet@unige.ch
}

\section{Table of Contents}

1. General information $\quad$ S2

$\begin{array}{lll}1.1 & \text { Substrate synthesis } & \text { S2 }\end{array}$

2. Rh-catalyzed isomerization of VPCs to conjugated dienoates S4

$\begin{array}{llr}2.1 & \text { Reaction optimization } & \text { S4 }\end{array}$

$\begin{array}{lll}2.2 & \text { Reaction scope } & \text { S5 }\end{array}$

$\begin{array}{lll}2.3 & \text { Data of new compounds } & \text { S6 }\end{array}$

3. Ru-catalyzed isomerization of VPCs to cross-conjugated dienoates $\mathbf{S 1 3}$

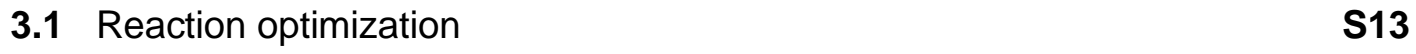

$\begin{array}{llr}3.2 & \text { Reaction scope } & \text { S15 }\end{array}$

$\begin{array}{ll}\text { 3.3 Data of new compounds } & \mathbf{S 1 6}\end{array}$

$\begin{array}{lll}\text { 5. } & \text { References } & \text { S26 }\end{array}$

6. NMR spectra of new compounds $\quad$ S27 


\section{General information}

Unless otherwise noted, all reactions were carried out under an inert atmosphere of nitrogen using either a two-manifold vacuum/inert gas Schlenk line or a M. Braun glovebox. Solvents were dried over activated alumina columns or distilled and then degassed by two freeze-pumpthaw cycles. Commercial reagents, precatalysts and ligands were purchased from Aldrich, Fluka, Acros or Strem and used without purification unless otherwise noted. NMR spectra were recorded on AMX-300 and AMX-400 Bruker Avance spectrometers at $298 \mathrm{~K} .{ }^{1} \mathrm{H}$ and ${ }^{13} \mathrm{C}\left\{{ }^{1} \mathrm{H}\right\}$ NMR chemical shifts are given in ppm relative to $\mathrm{SiMe}_{4}$, with the solvent resonance used as internal reference $\left(\mathrm{CHCl}_{3}\right.$ proton at $7.26,{ }^{13} \mathrm{C}$ at $\left.77.16 \mathrm{ppm}\right) \cdot{ }^{19} \mathrm{~F}\left\{{ }^{1} \mathrm{H}\right\}$ NMR chemical shifts are reported in ppm with absolute reference relative to ${ }^{1} \mathrm{H}$. HRMS data were obtained on a Xevo G2 Tof spectrometer (Ionization mode: ESI positive polarity; Mobile phase: $\mathrm{MeOH}$ $100 \mu \mathrm{l} / \mathrm{min}$ ). Mass spectrum was calibrated by the use of the MS lockspray system (LeuEnk calibration solution). Infrared spectra were obtained on a Perkin Elmer 1650 FT-IR spectrometer using neat samples on a diamond ATR Golden Gate sampler. Melting points were recorded on a Buchi SMP-20 melting point apparatus using open glass capillaries. X-ray data collection was performed with a Rigaku XtaLAB Synergy-S diffractometer equipped with a hybrid pixel HyPix-Arc $150^{\circ}$ detector using $\mathrm{Cu}[\mathrm{Ka}]$ radiation at $120 \mathrm{~K}$. Enantiomeric excesses were determined either by HPLC or SFC analyses. HPLC analyses were performed on a Shimadzu CTO-20AA with DAICEL OD-H, OJ-H, AD-H and IC columns. Thin layer chromatography (TLC) was performed on plates of silica precoated with $0.25 \mathrm{~mm}$ Kieselgel 60 F254 from Merck. Flash chromatography was performed using silica gel SiliaFlash® P60 (230-400 mesh) from Silicycle. When appropriate, all reactions were heated using an oil bath.

\subsection{Substrate synthesis}

All of the non-commercially available 2 -substituted-1,3 dienes $\mathbf{1 a - h}$ were obtained by $\mathrm{Ni}$ catalyzed vinylation of the corresponding enol phosphates according to reported protocols..$^{1,2}$ Cyclopropanation of these dienes was performed according to our previously reported coppercatalyzed procedure using 6,6'-dimethyl-2,2'-bipiridyl as ligand. ${ }^{3}$ Results are reported in FigureS1. The obtained crude mixtures were subjected to a quick flash-chromatography to remove decomposed catalyst and other impurities. VCPs $\mathbf{3 a - h}$ and $\mathbf{4 a - h}$ were collected as regio-diastereoisomeric mixtures. Diastereomeric and regioisomeric ratios were determined by ${ }^{1} \mathrm{H}$ NMR. The identities of the different VCP isomers were either assigned by analogy with the isolated model compounds $\mathbf{3 a}$ and $\mathbf{4 a}$ or by NOESY. 


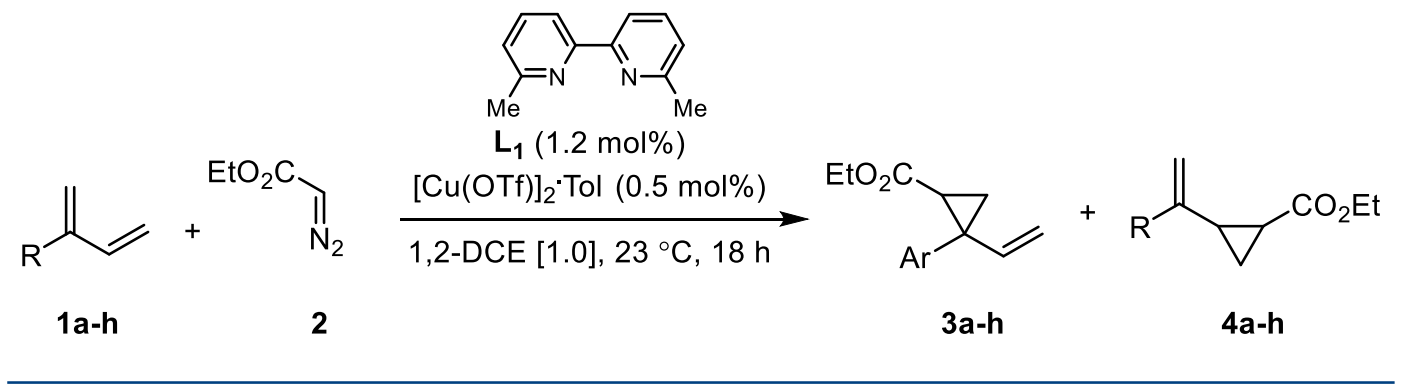<smiles>C=CC1(c2ccc(-c3ccccc3)cc2)C[C@H]1C(=O)OCC</smiles>

\section{$3 a$}

$65 \%$ yield cis/trans $=1.7: 1$ $r r_{(3 \mathbf{a} / 4 \mathbf{a})}=13.3: 1$

\section{$3 c$}<smiles>C=CC1(c2ccc(C(C)C)cc2)C[C@H]1COCC</smiles>

$61 \%$ yield cis/trans $=1.6: 1$ $r r_{(3 c / 4 c)}=14.3: 1$<smiles>C=CC1(c2ccc3ccccc3c2)CC1C(=O)OCC</smiles><smiles>C=CC1(c2ccsc2)CC1COCC</smiles>

\section{$3 e$}

$51 \%$ yield cis/trans $=1.4: 1$

$r r_{(3 \mathrm{e} / 4 \mathrm{e})}=9.3: 1$

\section{$3 \mathrm{~g}$}

$49 \%$ yield cis/trans $=1.1: 1$ $r r_{(3 \mathrm{~g} / \mathbf{4 g})}=9.3: 1$<smiles>C=CC1(c2ccc(OC)cc2)C[C@H]1COCC</smiles>

3b

$52 \%$ yield cis/trans $=1.2: 1$ $r r_{(3 \mathbf{b} / 4 \mathbf{b})}=14.3: 1$<smiles>C=CC1(c2ccc(C(F)(F)F)cc2)C[C@H]1COCC</smiles>

\section{3d}

$67 \%$ yield cis/trans $=1.7: 1$

$r r_{(3 d / 4 d)}=4.4: 1$<smiles>C=CC1(c2cn(C(=O)OC(C)(C)C)c3ccccc23)CC1C</smiles><smiles>C=CC1(CCc2ccccc2)CC1C(=O)OCC</smiles>

\section{$3 h$}

$51 \%$ yield cis/trans $=1.4: 1$ $r r_{(3 \mathrm{~h} / 4 \mathrm{~h})}=4.4: 1$

FigureS1. Scope of the Cu-catalyzed cyclopropanation. Selectivity determined by ${ }^{1} \mathrm{H}$ NMR. Yield after purification. 


\section{Rh-catalyzed isomerization of VPCs to conjugated dienoates}

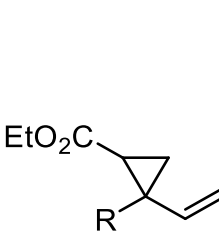

3a-h

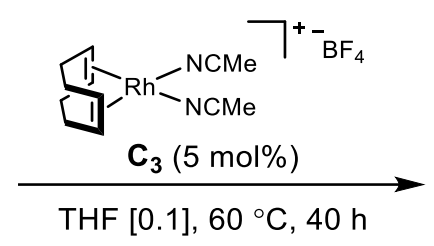

$\mathrm{HF}[0.1], 60^{\circ} \mathrm{C}, 40 \mathrm{~h}$

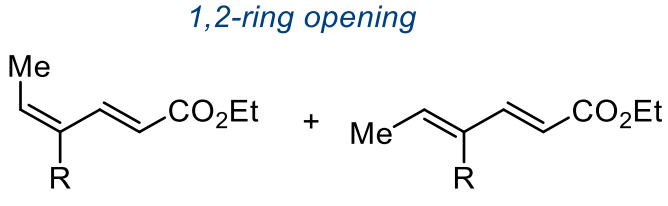

$(E, E)-\mathbf{5 a - h}$

$(E, Z)-\mathbf{5 a - h}$

FigureS2. Rh-catalyzed isomerization of VCPs to conjugated dienoates

\section{General procedure - A}

In a $5 \mathrm{~mL}$ flame-dried Schlenk tube, the mixture of vinylcyclopropanes $3 \mathbf{a}-\mathbf{h}(0.25 \mathrm{mmol})$ and $\left[\mathrm{Rh}\left(\mathrm{CH}_{3} \mathrm{CN}\right)_{2}(\mathrm{Cod})\right] \mathrm{BF}_{4} \mathrm{C}_{3}(0.0125 \mathrm{mmol})$ were dissolved in $2.5 \mathrm{~mL}$ of anhydrous and degassed tetrahydrofuran. The reaction mixture was stirred at $60^{\circ} \mathrm{C}$ for 40 hours.

\subsection{Reaction optimization}

Catalysts and reaction conditions were tested according to general procedure $\mathbf{A}$ using the mixture of $\mathbf{3 a}$ as model substrate $($ cis/trans $=1.7: 1, \operatorname{rr}(\mathbf{3 a} / \mathbf{a})=13.3: 1)$.

\begin{tabular}{cccccc}
\hline Precatalyst & Solvent & $\mathbf{T}\left({ }^{\circ} \mathbf{C}\right)$ & $\mathbf{t}(\mathbf{h})$ & Conv. $(\%)^{a}$ & $\mathbf{E , E} / \mathbf{E}, \mathbf{Z}$ \\
\hline$\left[\mathrm{Rh}(\mathrm{cod})_{2}\right] \mathrm{BF}_{4} \mathrm{C}_{6}$ & $1,2-\mathrm{DCE}$ & 60 & 20 & 90 & $3: 1$ \\
{$\left[\mathrm{Rh}(\mathrm{cod})\left(\mathrm{CH}_{3} \mathrm{CN}\right)_{2}\right] \mathrm{BF}_{4} \mathrm{C}_{3}$} & $1,2-\mathrm{DCE}$ & 60 & 20 & 89 & $6: 1$ \\
{$\left[\mathrm{Rh}(\mathrm{cod})\left(\mathrm{CH}_{3} \mathrm{CN}\right)_{2}\right] \mathrm{BF}_{4} \mathrm{C}_{3}$} & $1,2-\mathrm{DCE}$ & 80 & 2 & 93 & $4: 1$ \\
{$\left[\mathrm{Rh}(\mathrm{cod})\left(\mathrm{CH}_{3} \mathrm{CN}\right)_{2}\right] \mathrm{BF}_{4} \mathrm{C}_{3}$} & MeCN & 80 & 2 & n.r. ${ }^{b}$ & - \\
{$\left[\mathrm{Rh}(\mathrm{cod})\left(\mathrm{CH}_{3} \mathrm{CN}\right)_{2}\right] \mathrm{BF}_{4} \mathrm{C}_{3}$} & Toluene & 60 & 20 & n.r. ${ }^{b}$ & - \\
{$\left[\mathrm{Rh}(\mathrm{cod})\left(\mathrm{CH}_{3} \mathrm{CN}\right)_{2}\right] \mathrm{BF}_{4} \mathrm{C}_{3}$} & THF & 60 & 20 & 86 & $>20: 1$ \\
{$\left[\mathrm{Rh}(\mathrm{cod})\left(\mathrm{CH}_{3} \mathrm{CN}\right)_{2}\right] \mathrm{BF}_{4} \mathrm{C}_{3}$} & THF & 60 & 40 & $93(93)^{c}$ & $>20: 1$ \\
\hline
\end{tabular}

Table S1. Optimization of Rh-catalyzed ring opening. a Determined by ${ }^{1} \mathrm{H}$ NMR using mesitylene as internal standard. ${ }^{b}$ No reaction. ${ }^{c}$ Isolated yield after purification. 


\subsection{Reaction scope}

The VCPs obtained by diene cyclopropanation were subjected to general procedure $\mathbf{A}$ Results are reported in FigureS3.

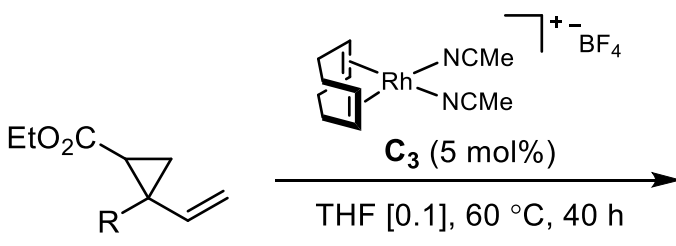

cis/trans-3a-h

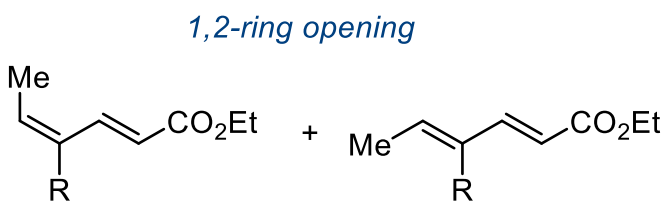

$(E, E)-5 \mathbf{a}-\mathbf{h}$
$(E, Z)-5 a-h$<smiles>CC=C(C=CC(=O)OCC)c1ccc(-c2ccccc2)cc1</smiles><smiles>CC=C(C=CC(=O)OCC)c1ccc(OC)cc1</smiles><smiles>CC=C(C=CC(=O)OCC)c1ccc(Br)cc1</smiles><smiles>CC=C(C=CC(OCC)C(F)(F)C(F)(F)C(F)(F)C(F)(F)F)c1ccc(C(F)(F)F)cc1</smiles><smiles>C/C=C(\C=C\C(=O)OCC)c1ccc2ccccc2c1</smiles><smiles>CC=C(C=CC(=O)OCC)c1cn(C(=O)OC(C)(C)C)c2ccccc12</smiles><smiles>CC=C(C=CC(=O)OCC)c1ccsc1</smiles><smiles>C/C=C(\C=C\C(=O)OCC)CCc1ccccc1</smiles>

$(E, E) /(E, Z)>20: 1$

Figure S3. Scope of the Rh-catalyzed ring opening. Selectivity determined by ${ }^{1} \mathrm{H}$ NMR. Yield after purification. 


\subsection{Data of new compounds}

\section{Ethyl $(2 E, 4 E)-4-\left(\left[1,1^{\prime}-\right.\right.$ biphenyl]-4-yl)hexa-2,4-dienoate $(E, E)-5 a$}<smiles>C/C=C(\C=C\C(=O)OCC(C)(F)F)c1ccc(-c2ccccc2)cc1</smiles>

Synthesized according to general procedure A starting from a racemic mixture of $\mathbf{3 a}$ and $\mathbf{4 a}$ isomers. Obtained as an off-white solid after flash chromatography using pentane/ $\mathrm{Et}_{2} \mathrm{O} 96: 4$ as eluent $(68 \mathrm{mg}, 93 \%$ yield, $>20: 1$ $(E, E) /(E, Z), 90 \%$ purity). The VCP mixture was obtained according to the general cyclopropanation procedure $(13.3: 1 \mathrm{rr}$ (3a/4a) $=, 1.7: 1 \mathrm{dr}$ (cis/trans) $)$.

${ }^{1} \mathrm{H}$ NMR $\left(\mathrm{CDCl}_{3}, 400 \mathrm{MHz}\right) \delta(\mathrm{ppm})=7.94\left(\mathrm{~d},{ }^{3} \mathrm{~J}_{\mathrm{H}-\mathrm{H}}=15.7 \mathrm{~Hz}, 1 \mathrm{H}, \mathrm{H}-4\right), 7.63-7.59(\mathrm{~m}, 2 \mathrm{H}, \mathrm{H}-$ 14), 7.59-7.54 (m, 2H, H-11), 7.49-7.41 (m, 2H, H-15), 7.39-7.32 (t, $\left.{ }^{3} \mathrm{~J}_{\mathrm{H}-\mathrm{H}}=7.4 \mathrm{~Hz}, 1 \mathrm{H}, \mathrm{H}-16\right)$, $7.29\left(\mathrm{~d},{ }^{3} \mathrm{~J}_{\mathrm{H}-\mathrm{H}}=8.2 \mathrm{~Hz}, 2 \mathrm{H}, \mathrm{H}-10\right), 6.02\left(\mathrm{q},{ }^{3} \mathrm{~J}_{\mathrm{H}-\mathrm{H}}=7.3 \mathrm{~Hz}, 1 \mathrm{H}, \mathrm{H}-2\right), 5.82\left(\mathrm{~d},{ }^{3} \mathrm{~J}_{\mathrm{H}-\mathrm{H}}=15.7 \mathrm{~Hz}\right.$, $1 \mathrm{H}, \mathrm{H}-5), 4.22\left(\mathrm{q},{ }^{3} \mathrm{~J}_{\mathrm{H}-\mathrm{H}}=7.1 \mathrm{~Hz}, 2 \mathrm{H}, \mathrm{H}-7\right), 2.05\left(\mathrm{~d},{ }^{3} \mathrm{~J}_{\mathrm{H}-\mathrm{H}}=7.3 \mathrm{~Hz}, 3 \mathrm{H}, \mathrm{H}-1\right), 1.29\left(\mathrm{t},{ }^{3} \mathrm{~J}_{\mathrm{H}-\mathrm{H}}=7.1\right.$ $\mathrm{Hz}, 3 \mathrm{H}, \mathrm{H}-8)$

${ }^{13} \mathrm{C}\left\{{ }^{1} \mathrm{H}\right\}$ NMR $\left(\mathrm{CDCl}_{3}, 101 \mathrm{MHz}\right) \delta(\mathrm{ppm})=167.7(\mathrm{C}-6), 140.9(\mathrm{C}-13), 140.3(\mathrm{C}-12), 140.3(\mathrm{C}-$ 4), 139.8 (C-9), 138.8 (C-3), 135.3 (C-2), 129.3 (C-10), 128.9 (C-15), 127.4 (C-16), 127.2 (C14), 127.2 (C-11), 121.3 (C-5), 60.5 (C-7), 14.6 (C-1), 14.4 (C-8)

MP $\left({ }^{\circ} \mathrm{C}\right)=306-309$

HRMS $\left(\mathrm{ESI}^{+}\right)$calculated for $\mathrm{C}_{20} \mathrm{H}_{21} \mathrm{O}_{2}[\mathrm{M}+\mathrm{H}]^{+}=293.1536$; found $=293.1520$

IR (neat) $\vee\left(\mathrm{cm}^{-1}\right)=2984,1710,1621,1295,1268,1171,1035,849$

\section{Ethyl (2E,4E)-4-(4-methoxyphenyl)hexa-2,4-dienoate $(E, E)-5 \mathbf{b}$}

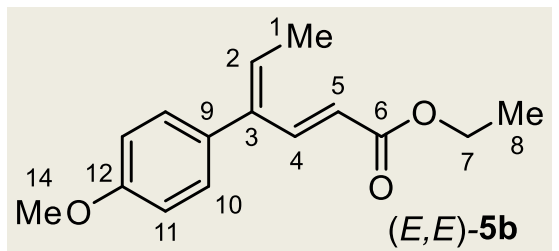

$(E, E) /(E, Z), 95 \%$ purity). The VCP mixture was obtained according to the general

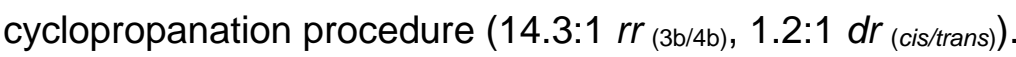

${ }^{1} \mathrm{H}$ NMR $\left(\mathrm{CDCl}_{3}, 400 \mathrm{MHz}\right) \delta(\mathrm{ppm})=7.90\left(\mathrm{dd},{ }^{3} \mathrm{~J}_{\mathrm{H}-\mathrm{H}}=15.7 \mathrm{~Hz},{ }^{4} \mathrm{~J}_{\mathrm{H}-\mathrm{H}}=0.7 \mathrm{~Hz}, 1 \mathrm{H}, \mathrm{H}-4\right), 7.16-$ $7.10(\mathrm{~m}, 2 \mathrm{H}, \mathrm{H}-10), 6.90-6.84(\mathrm{~m}, 2 \mathrm{H}, \mathrm{H}-11), 5.92\left(\mathrm{q},{ }^{3} \mathrm{~J}_{\mathrm{H}-\mathrm{H}}=7.3 \mathrm{~Hz}, 1 \mathrm{H}, \mathrm{H}-2\right), 5.75\left(\mathrm{~d},{ }^{3} \mathrm{~J}_{\mathrm{H}-\mathrm{H}}=\right.$ $15.7 \mathrm{~Hz}, 1 \mathrm{H}, \mathrm{H}-5), 4.20\left(\mathrm{q},{ }^{3} \mathrm{~J}_{\mathrm{H}-\mathrm{H}}=7.1 \mathrm{~Hz}, 2 \mathrm{H}, \mathrm{H}-7\right), 3.82(\mathrm{~s}, 3 \mathrm{H}, \mathrm{H}-14), 2.00\left(\mathrm{~d},{ }^{3} \mathrm{~J}_{\mathrm{H}-\mathrm{H}}=7.3 \mathrm{~Hz}\right.$, $3 \mathrm{H}, \mathrm{H}-1), 1.28\left(\mathrm{t},{ }^{3} \mathrm{~J}_{\mathrm{H}-\mathrm{H}}=7.1,3 \mathrm{H}, \mathrm{H}-8\right)$ 
${ }^{13} \mathrm{C}\left\{{ }^{1} \mathrm{H}\right\}$ NMR $\left(\mathrm{CDCl}_{3}, 101 \mathrm{MHz}\right) \delta(\mathrm{ppm})=167.8(\mathrm{C}-6), 159.0(\mathrm{C}-12), 140.7(\mathrm{C}-4), 138.7$ (C3), 134.7 (C-2), 133.2 (C-9), 129.9 (C-10), 121.1 (C-5), 113.8 (C-11), 60.4 (C-7), 55.4 (C-14), $14.5(\mathrm{C}-1), 14.4(\mathrm{C}-8)$

HRMS $\left(\mathrm{ESI}^{+}\right)$calculated for $\mathrm{C}_{15} \mathrm{H}_{19} \mathrm{O}_{3}[\mathrm{M}+\mathrm{H}]^{+}=247.1329$; found $=247.1312$

IR (neat) $\vee\left(\mathrm{cm}^{-1}\right)=2933,1717,1608,1510,1244,1141,1031,832$

\section{Ethyl (2E,4E)-4-(4-isobutylphenyl)hexa-2,4-dienoate $(E, E)-5 \mathrm{c}$}

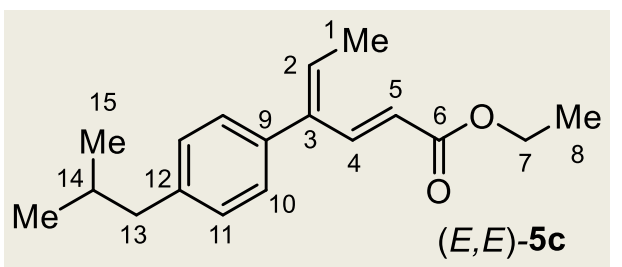

$(E, E)-5 c$

Synthesized according to general procedure A starting from a racemic mixture of $\mathbf{3 c}$ and $\mathbf{4 c}$ isomers. Obtained as a yellowish oil after flash chromatography using pentane/ $\mathrm{Et}_{2} \mathrm{O} 98.5: 1.5$ as eluent $(58.6 \mathrm{mg}, 86 \%$ yield, $>20: 1(E, E) /(E, Z), 98 \%$ purity). The VCP mixture was obtained according to the general cyclopropanation procedure (14.3:1 $\left.r r_{(3 \mathrm{c} / 4 \mathrm{c})}, 2.4: 1 d r_{(\text {cis/trans) }}\right)$.

$4 \mathrm{mmol}$ scale reaction: $1.1 \mathrm{~g}, 99 \%$ yield, $>20: 1(E, E) /(E, Z)$

${ }^{1} \mathrm{H}$ NMR $\left(\mathrm{CDCl}_{3}, 400 \mathrm{MHz}\right) \delta(\mathrm{ppm})=7.91\left(\mathrm{dd},{ }^{3} \mathrm{~J}_{\mathrm{H}-\mathrm{H}}=15.7 \mathrm{~Hz},{ }^{4} \mathrm{~J}_{\mathrm{H}-\mathrm{H}}=0.9 \mathrm{~Hz}, 1 \mathrm{H}, \mathrm{H}-4\right), 7.11$ (s, 4H, H-10+H-11), 5.95 (q, $\left.{ }^{3} \mathrm{~J}_{\mathrm{H}-\mathrm{H}}=7.3 \mathrm{~Hz}, 1 \mathrm{H}, \mathrm{H}-2\right), 5.77$ (d, $\left.{ }^{3} \mathrm{~J}_{\mathrm{H}-\mathrm{H}}=15.7 \mathrm{~Hz}, 1 \mathrm{H}, \mathrm{H}-5\right), 4.20$ (q, ${ }^{3} \mathrm{~J}_{\mathrm{H}-\mathrm{H}}=7.1 \mathrm{~Hz}, 2 \mathrm{H}, \mathrm{H}-7$ ), $2.47\left(\mathrm{~d},{ }^{3} \mathrm{~J}_{\mathrm{H}-\mathrm{H}}=7.2 \mathrm{~Hz}, 2 \mathrm{H}, \mathrm{H}-13\right), 2.01\left(\mathrm{~d},{ }^{3} \mathrm{~J}_{\mathrm{H}-\mathrm{H}}=7.3 \mathrm{~Hz}, 3 \mathrm{H}, \mathrm{H}-\right.$ 1), $1.95-1.80(\mathrm{~m}, 1 \mathrm{H}, \mathrm{H}-14), 1.28\left(\mathrm{t},{ }^{3} \mathrm{~J}_{\mathrm{H}-\mathrm{H}}=7.1 \mathrm{~Hz}, 3 \mathrm{H}, \mathrm{H}-8\right), 0.92\left(\mathrm{~d},{ }^{3} \mathrm{~J}_{\mathrm{H}-\mathrm{H}}=6.6 \mathrm{~Hz}, 6 \mathrm{H}, \mathrm{H}-15\right)$

${ }^{13} \mathrm{C}\left\{{ }^{1} \mathrm{H}\right\}$ NMR $\left(\mathrm{CDCl}_{3}, 101 \mathrm{MHz}\right) \delta(\mathrm{ppm})=167.8(\mathrm{C}-6), 140.9(\mathrm{C}-12), 140.5(\mathrm{C}-4), 139.1$ (C3), 138.1 (C-9), 134.9 (C-2), 129.1 (C-11), 128.5 (C-10), 121.0 (C-5), 60.5 (C-7), 45.3 (C-13), 30.4 (C-14), 22.6 (C-15), 14.6 (C-1), 14.5 (C-8)

HRMS $\left(\mathrm{ESI}^{+}\right)$calculated for $\mathrm{C}_{18} \mathrm{H}_{25} \mathrm{O}_{2}[\mathrm{M}+\mathrm{H}]^{+}=273.1850$; found $=273.1840$

IR $($ neat $) \vee\left(\mathrm{cm}^{-1}\right)=2956,1713,1625,1266,1164,1042,982,873$

\section{Ethyl $(2 E, 4 E)$-4-(4-(trifluoromethyl)phenyl)hexa-2,4-dienoate $(E, E)-5 \mathbf{d}$}

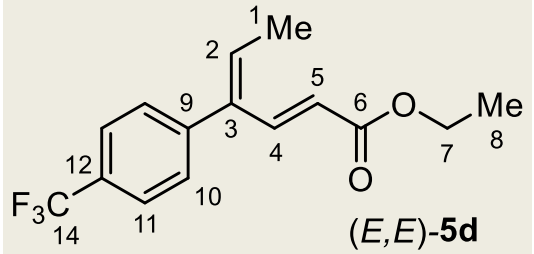

$(E, E)-\mathbf{5 d}$
Synthesized according to general procedure A starting from a racemic mixture of $\mathbf{3} \mathbf{d}$ and $\mathbf{4 d}$ isomers. Obtained as a yellowish oil after flash chromatography using pentane/Et $\mathrm{t}_{2} \mathrm{O}$ 99.3:0.7 as eluent $(46.9 \mathrm{mg}, 66 \%$ yield, $>20: 1(E, E) /(E, Z)$, $93 \%$ purity). The VCP mixture was obtained according to the general cyclopropanation procedure $\left(4.4: 1 \mathrm{rr}{ }_{(3 \mathrm{~d} / 4 \mathrm{~d})}, 1.7: 1 \mathrm{dr}\right.$ (cis/trans) $)$. 
${ }^{1} \mathrm{H}$ NMR $\left(\mathrm{CDCl}_{3}, 400 \mathrm{MHz}\right) \delta(\mathrm{ppm})=7.90\left(\mathrm{~d},{ }^{3} \mathrm{~J}_{\mathrm{H}-\mathrm{H}}=15.8,1 \mathrm{H}, \mathrm{H}-4\right), 7.60\left(\mathrm{~d},{ }^{3} \mathrm{~J}_{\mathrm{H}-\mathrm{H}}=8.1 \mathrm{~Hz}\right.$, $2 \mathrm{H}, \mathrm{H}-11), 7.33\left(\mathrm{~d},{ }^{3} \mathrm{~J}_{\mathrm{H}-\mathrm{H}}=8.0 \mathrm{~Hz}, 2 \mathrm{H}, \mathrm{H}-10\right), 5.98\left(\mathrm{q},{ }^{3} \mathrm{~J}_{\mathrm{H}-\mathrm{H}}=7.3,1 \mathrm{H}, \mathrm{H}-1\right), 5.67\left(\mathrm{~d},{ }^{3} \mathrm{~J}_{\mathrm{H}-\mathrm{H}}=\right.$ 15.8, 1H, H-5), 4.21 (q, ${ }^{3} \mathrm{~J}_{\mathrm{H}-\mathrm{H}}=7.1,2 \mathrm{H}, \mathrm{H}-7$ ), 2.05 (d, 1 (q, $\left.{ }^{3} \mathrm{~J}_{\mathrm{H}-\mathrm{H}}=7.3 \mathrm{~Hz}, 3 \mathrm{H}, \mathrm{H}-1\right), 1.28$ (t, $\left.{ }^{3} \mathrm{~J}_{\mathrm{H}-\mathrm{H}}=7.1 \mathrm{~Hz}, 3 \mathrm{H}, \mathrm{H}-8\right)$

${ }^{13} \mathrm{C}\left\{{ }^{1} \mathrm{H}\right\}$ NMR $\left(\mathrm{CDCl}_{3}, 101 \mathrm{MHz}\right) \delta(\mathrm{ppm})=167.4(\mathrm{C}-6), 144.5(\mathrm{C}-9), 139.6$ (C-4), $138.2(\mathrm{C}-3)$, $136.3(\mathrm{C}-3), 129.7$ (q, $\left.{ }^{2} \mathrm{~J}_{\mathrm{F}-\mathrm{H}}=32.4 \mathrm{~Hz}, \mathrm{C}-12\right), 129.2$ (C-10), 125.4 (q, ${ }^{3} \mathrm{~J}_{\mathrm{F}-\mathrm{H}}=32.4 \mathrm{~Hz}, \mathrm{C}-11$ ), $124.3\left(\mathrm{q},{ }^{1} \mathrm{~J}_{\mathrm{F}-\mathrm{H}}=272.7 \mathrm{~Hz}, \mathrm{C}-14\right), 121.5(\mathrm{C}-5), 60.6(\mathrm{C}-7), 14.6$ (C-1), 14.4 (C-8)

${ }^{19} \mathrm{~F}\left\{{ }^{1} \mathrm{H}\right\} \operatorname{NMR}\left(\mathrm{CDCl}_{3}, 282 \mathrm{MHz}\right) \delta(\mathrm{ppm})=-62.5$

MP $\left({ }^{\circ} \mathrm{C}\right)=288-292$

HRMS $\left(\mathrm{ESI}^{+}\right)$calculated for $\mathrm{C}_{15} \mathrm{H}_{16} \mathrm{O}_{2} \mathrm{~F}_{3}[\mathrm{M}+\mathrm{H}]^{+}=285.1097$; found $=285.1107$

IR $($ neat $) \vee\left(\mathrm{cm}^{-1}\right)=2922,1728,1616,1328,1169,1099,841$

Ethyl (2E,4E)-4-(naphthalen-2-yl)hexa-2,4-dienoate $(E, E)-5 \mathbf{e}$

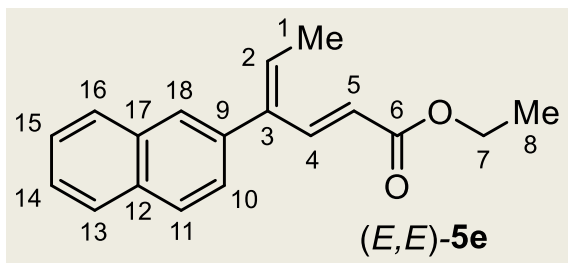

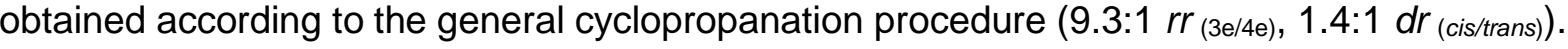

${ }^{1} \mathrm{H}$ NMR $\left(\mathrm{CDCl}_{3}, 400 \mathrm{MHz}\right) \delta(\mathrm{ppm})=7.99\left(\mathrm{dd},{ }^{3} \mathrm{~J}_{\mathrm{H}-\mathrm{H}}=15.7 \mathrm{~Hz},{ }^{4} \mathrm{~J}_{\mathrm{H}-\mathrm{H}}=0.6 \mathrm{~Hz}, 1 \mathrm{H}, \mathrm{H}-4\right), 7.88-$ $7.77(\mathrm{~m}, 3 \mathrm{H}, \mathrm{H}-11+\mathrm{H}-13+\mathrm{H}-16), 7.69-7.67(\mathrm{~m}, 1 \mathrm{H}, \mathrm{H}-18), 7.51-7.45(\mathrm{~m}, 2 \mathrm{H}, \mathrm{H}-14+\mathrm{H}-15), 7.34$ $\left(\mathrm{dd},{ }^{3} \mathrm{~J}_{\mathrm{H}-\mathrm{H}}=8.4,{ }^{4} \mathrm{~J}_{\mathrm{H}-\mathrm{H}}=1.7,1 \mathrm{H}, \mathrm{H}-10\right), 6.07\left(\mathrm{q},{ }^{3} \mathrm{~J}_{\mathrm{H}-\mathrm{H}}=7.3,1 \mathrm{H}, \mathrm{H}-2\right), 5.76\left(\mathrm{~d},{ }^{3} \mathrm{~J}_{\mathrm{H}-\mathrm{H}}=15.7,1 \mathrm{H}\right.$, $\mathrm{H}-5), 4.20\left(\mathrm{q},{ }^{3} \mathrm{~J}_{\mathrm{H}-\mathrm{H}}=7.1 \mathrm{~Hz}, 2 \mathrm{H}, \mathrm{H}-7\right), 2.08\left(\mathrm{~d},{ }^{3} \mathrm{~J}_{\mathrm{H}-\mathrm{H}}=7.3 \mathrm{~Hz}, 3 \mathrm{H}, \mathrm{H}-1\right), 1.27\left(\mathrm{t},{ }^{3} \mathrm{~J}_{\mathrm{H}-\mathrm{H}}=7.1 \mathrm{~Hz}\right.$, $3 \mathrm{H}, \mathrm{H}-8)$

${ }^{13} \mathrm{C}\left\{{ }^{1} \mathrm{H}\right\}$ NMR $\left(\mathrm{CDCl}_{3}, 101 \mathrm{MHz}\right) \delta(\mathrm{ppm})=167.7(\mathrm{C}-6), 140.4(\mathrm{C}-4), 139.2(\mathrm{C}-3), 138.4(\mathrm{C}-9)$, 135.7 (C-2), 133.5 (C-17), 132.8 (C-12), 128.1 (C-11/C13/C-16), 127.8 (C-11/C13/C-16), 127.8 (C-11/C13/C-16), 127.5 (C-18), 127.2 (C-10), 126.3 (C-14/C-15) , 126.1 (C-14/C-15), 121.5 (C-5), 60.5 (C-7), 14.7 (C-1), 14.4 (C-8)

$\operatorname{MP}\left({ }^{\circ} \mathrm{C}\right)=66-70$

HRMS $\left(\mathrm{ESI}^{+}\right)$calculated for $\mathrm{C}_{18} \mathrm{H}_{19} \mathrm{O}_{2}[\mathrm{M}+\mathrm{H}]^{+}=267.1380 ;$ found $=267.1391$

IR $($ neat $) \vee\left(\mathrm{cm}^{-1}\right)=2980,1711,1618,1265,1167,1040,861$ 
tert-Butyl 3-((2E,4E)-6-ethoxy-6-oxohexa-2,4-dien-3-yl)-indole-1-carboxylate $(E, E)-5 \mathbf{f}$

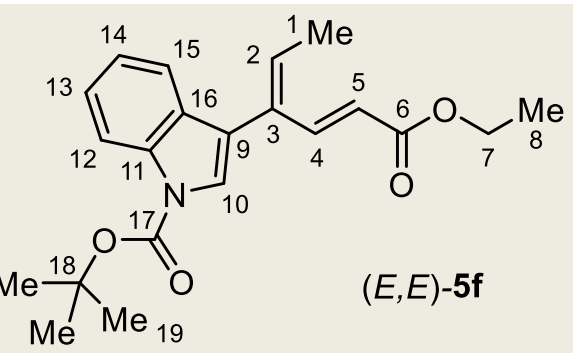

Synthesized according to general procedure A starting from a racemic mixture of $\mathbf{3} \mathbf{f}$ and $\mathbf{4} \mathbf{f}$ isomers. Obtained as a yellowish solid after flash chromatography using pentane/ $\mathrm{Et}_{2} \mathrm{O} 97.5: 2.5$ as eluent $(60.4 \mathrm{mg}, 68 \%$ yield, $18: 1(E, E) /(E, Z), 95 \%$ purity). The VCP mixture was obtained according to the general cyclopropanation

procedure $\left(8: 1 r r_{(3 / / 4 f)}, 1.9: 1 d r_{(\text {cis/trans })}\right)$.

${ }^{1} \mathrm{H}$ NMR $\left(\mathrm{CDCl}_{3}, 400 \mathrm{MHz}\right) \delta(\mathrm{ppm})=8.15\left(\mathrm{~d},{ }^{3} \mathrm{~J}_{\mathrm{H}-\mathrm{H}}=8.0 \mathrm{~Hz}, 1 \mathrm{H}, \mathrm{H}-12\right), 7.96\left(\mathrm{dd},{ }^{3} \mathrm{~J}_{\mathrm{H}-\mathrm{H}}=15.6\right.$ $\left.\mathrm{Hz},{ }^{4} \mathrm{~J}_{\mathrm{H}-\mathrm{H}}=0.8 \mathrm{~Hz}, 1 \mathrm{H}, \mathrm{H}-4\right), 7.48(\mathrm{~s}, 1 \mathrm{H}, \mathrm{H}-10), 7.41-7.36(\mathrm{~m}, 1 \mathrm{H}, \mathrm{H}-15), 7.31$ (ddd, ${ }^{3} \mathrm{~J}_{\mathrm{H}-\mathrm{H}}=8.4$ $\left.\mathrm{Hz},{ }^{3} \mathrm{~J}_{\mathrm{H}-\mathrm{H}}=7.2 \mathrm{~Hz},{ }^{4} \mathrm{~J}_{\mathrm{H}-\mathrm{H}}=1.3 \mathrm{~Hz}, 1 \mathrm{H}, \mathrm{H}-13\right), 7.20\left(\mathrm{ddd},{ }^{3} \mathrm{~J}_{\mathrm{H}-\mathrm{H}}=8.0 \mathrm{~Hz},{ }^{3} \mathrm{~J}_{\mathrm{H}-\mathrm{H}}=7.2 \mathrm{~Hz},{ }^{3} \mathrm{~J}_{\mathrm{H}-\mathrm{H}}=\right.$ $1.1 \mathrm{~Hz}, 1 \mathrm{H}, \mathrm{H}-14), 6.13\left(\mathrm{q},{ }^{3} \mathrm{~J}_{\mathrm{H}-\mathrm{H}}=7.3,1 \mathrm{H}, \mathrm{H}-2\right), 5.79\left(\mathrm{~d},{ }^{3} \mathrm{~J}_{\mathrm{H}-\mathrm{H}}=15.6 \mathrm{~Hz}, 1 \mathrm{H}, \mathrm{H}-5\right), 4.18$ (q, $\left.{ }^{3} \mathrm{~J}_{\mathrm{H}-\mathrm{H}}=7.1 \mathrm{~Hz}, 2 \mathrm{H}, \mathrm{H}-7\right), 2.08\left(\mathrm{~d},{ }^{3} \mathrm{~J}_{\mathrm{H}-\mathrm{H}}=7.3 \mathrm{~Hz}, 3 \mathrm{H}, \mathrm{H}-1\right), 1.25\left(\mathrm{t},{ }^{3} \mathrm{~J}_{\mathrm{H}-\mathrm{H}}=7.1 \mathrm{~Hz}, 3 \mathrm{H}, \mathrm{H}-8\right)$

${ }^{13} \mathrm{C}\left\{{ }^{1} \mathrm{H}\right\}$ NMR $\left(\mathrm{CDCl}_{3}, 101 \mathrm{MHz}\right) \delta(\mathrm{ppm})=167.7(\mathrm{C}-6), 139.8(\mathrm{C}-4), 136.7(\mathrm{C}-2), 135.3(\mathrm{C}-$ 11), 130.5 (C-3), 130.0 (C-16), 124.6 (C-13) 124.0 (C-10), 122.8 (C-14), 121.2 (C-5), 120.8 (C-9), 120.4 (C-15), 115.4 (C-12), 83.9 (C-18), 60.5 (C-7), 28.4 (C-19), 14.6 (C-1), 14.4 (C-8) MP $\left({ }^{\circ} \mathrm{C}\right)=90-94$

HRMS $\left(\mathrm{ESI}^{+}\right)$calculated for $\mathrm{C}_{21} \mathrm{H}_{26} \mathrm{NO}_{4}[\mathrm{M}+\mathrm{H}]^{+}=356.1857$; found $=356.1841$

IR (neat) $v\left(\mathrm{~cm}^{-1}\right)=2926,1728,1369,1244,1152$

\section{Ethyl (2E,4E)-4-(thiophen-3-yl)hexa-2,4-dienoate $(E, E)-5 \mathbf{g}$}

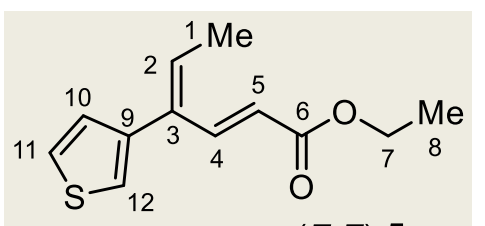

$(E, E)-\mathbf{5 g}$

Synthesized according to general procedure A starting from a racemic mixture of $\mathbf{3} \mathbf{g}$ and $\mathbf{4 g}$ isomers. Obtained as a yellowish oil after flash chromatography using pentane/ $\mathrm{Et}_{2} \mathrm{O} \quad 96: 4$ as eluent (49.5 mg, 89\% yield, >20:1 (E,E)/(E,Z), 98\% purity). The VCP mixture was obtained according to the general

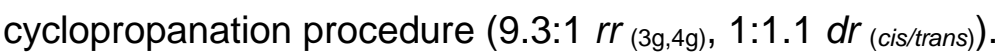

${ }^{1} \mathrm{H}$ NMR $\left(\mathrm{CDCl}_{3}, 400 \mathrm{MHz}\right) \delta(\mathrm{ppm})=7.86\left(\mathrm{dd},{ }^{3} \mathrm{~J}_{\mathrm{H}-\mathrm{H}}=15.7 \mathrm{~Hz},{ }^{4} \mathrm{~J}_{\mathrm{H}-\mathrm{H}}=0.6 \mathrm{~Hz}, 1 \mathrm{H}, \mathrm{H}-4\right), 7.29$ $\left(\mathrm{dd},{ }^{3} \mathrm{~J}_{\mathrm{H}-\mathrm{H}}=5.0 \mathrm{~Hz},{ }^{4} \mathrm{~J}_{\mathrm{H}-\mathrm{H}}=3.0 \mathrm{~Hz}, 1 \mathrm{H}, \mathrm{H}-11\right), 7.12\left(\mathrm{dd},{ }^{4} \mathrm{~J}_{\mathrm{H}-\mathrm{H}}=3.0 \mathrm{~Hz},{ }^{4} \mathrm{~J}_{\mathrm{H}-\mathrm{H}}=1.3 \mathrm{~Hz}, 1 \mathrm{H}, \mathrm{H}-\right.$ 12), $7.01\left(\mathrm{dd},{ }^{3} \mathrm{~J}_{\mathrm{H}-\mathrm{H}}=5.0 \mathrm{~Hz},{ }^{4} \mathrm{~J}_{\mathrm{H}-\mathrm{H}}=1.3 \mathrm{~Hz}, 1 \mathrm{H}, \mathrm{H}-10\right), 6.06\left(\mathrm{q},{ }^{3} \mathrm{~J}_{\mathrm{H}-\mathrm{H}}=7.3 \mathrm{~Hz}, 1 \mathrm{H}, \mathrm{H}-2\right), 5.89$ $\left(\mathrm{d},{ }^{3} \mathrm{~J}_{\mathrm{H}-\mathrm{H}}=15.7 \mathrm{~Hz}, 1 \mathrm{H}, \mathrm{H}-5\right), 4.21\left(\mathrm{q},{ }^{3} \mathrm{~J}_{\mathrm{H}-\mathrm{H}}=7.1 \mathrm{~Hz}, 2 \mathrm{H}, \mathrm{H}-7\right), 2.00\left(\mathrm{~d},{ }^{3} \mathrm{~J}_{\mathrm{H}-\mathrm{H}}=7.3 \mathrm{~Hz}, 1 \mathrm{H}, \mathrm{H}-\right.$ 1), $1.29\left(\mathrm{t},{ }^{3} \mathrm{~J}_{\mathrm{H}-\mathrm{H}}=7.1 \mathrm{~Hz}, 3 \mathrm{H}, \mathrm{H}-8\right)$ 
${ }^{13} \mathrm{C}\left\{{ }^{1} \mathrm{H}\right\}$ NMR $\left(\mathrm{CDCl}_{3}, 101 \mathrm{MHz}\right) \delta(\mathrm{ppm})=167.7(\mathrm{C}-6), 141.2(\mathrm{C}-9), 140.2(\mathrm{C}-4), 134.8(\mathrm{C}-2)$, 133.8 (C-3), 128.1 (C-10), 125.4 (C-11), 122.6 (C-12), 121.1 (C-5), 60.5 (C-7), 15.5 (C-1), 15.4 $(\mathrm{C}-8)$

HRMS $\left(\mathrm{ESI}^{+}\right)$calculated for $\mathrm{C}_{12} \mathrm{H}_{15} \mathrm{O}_{2} \mathrm{~S}[\mathrm{M}+\mathrm{H}]^{+}=223.0788$; found $=223.0797$

IR (neat) $\vee\left(\mathrm{cm}^{-1}\right)=2980,1710,1624,1275,1162,1034,856$

\section{Ethyl (2E,4Z)-4-phenethylhexa-2,4-dienoate $(E, E)-5 \mathrm{~h}$}<smiles>CC=CC(=CC=CC(=O)OCC)CCc1ccccc1</smiles>

$(E, E)-5 h$

Synthesized according to general procedure A starting from a racemic mixture of $3 \mathrm{~h}$ and $\mathbf{4 h}$ isomers. Obtained as a colorless oil after flash chromatography using pentane/ $\mathrm{Et}_{2} \mathrm{O} 98: 2$ as eluent $(26.3 \mathrm{mg}, 43 \%$ yield, $>20: 1$ $(E, E) /(E, Z), 92 \%$ purity). The VCP mixture was obtained according to the general cyclopropanation procedure $\left(4.4: 1 \mathrm{rr}_{(3 \mathrm{~h} / 4 \mathrm{~h})}, 1.4: 1 \mathrm{dr}\right.$ (cis/trans) $\left.)\right)$.

${ }^{1} \mathrm{H}$ NMR $\left(\mathrm{CDCl}_{3}, 400 \mathrm{MHz}\right) \delta(\mathrm{ppm})=7.76\left(\mathrm{dd},{ }^{3} \mathrm{~J}_{\mathrm{H}-\mathrm{H}}=15.9 \mathrm{~Hz},{ }^{4} \mathrm{~J}_{\mathrm{H}-\mathrm{H}}=0.9 \mathrm{~Hz}, 1 \mathrm{H}, \mathrm{H}-4\right), 7.32-$ $7.27(\mathrm{~m}, 2 \mathrm{H}, \mathrm{H}-13), 7.23-7.15(\mathrm{~m}, 3 \mathrm{H}, \mathrm{H} 12+\mathrm{H}-14), 5.99\left(\mathrm{~d},{ }^{3} \mathrm{~J}_{\mathrm{H}-\mathrm{H}}=15.9 \mathrm{~Hz}, 1 \mathrm{H}, \mathrm{H}-5\right), 4.25$ (q, $\left.{ }^{3} \mathrm{~J}_{\mathrm{H}-\mathrm{H}}=7.1 \mathrm{~Hz}, 2 \mathrm{H}, \mathrm{H}-7\right), 2.80-2.72(\mathrm{~m}, 2 \mathrm{H}, \mathrm{H}-10), 2.55-2.47(\mathrm{~m}, 2 \mathrm{H}, \mathrm{H}-9), 1.86\left(\mathrm{~d},{ }^{3} \mathrm{~J}_{\mathrm{H}-\mathrm{H}}=7.2\right.$ $\mathrm{Hz}, 3 \mathrm{H}, \mathrm{H}-1), 1.33\left(\mathrm{t},{ }^{3} \mathrm{~J}_{\mathrm{H}-\mathrm{H}}=7.1 \mathrm{~Hz}, 3 \mathrm{H}, \mathrm{H}-8\right)$

${ }^{13} \mathrm{C}\left\{{ }^{1} \mathrm{H}\right\}$ NMR $\left(\mathrm{CDCl}_{3}, 101 \mathrm{MHz}\right) \delta(\mathrm{ppm})=167.8(\mathrm{C}-6), 141.9(\mathrm{C}-11), 140.1(\mathrm{C}-4), 135.2(\mathrm{C}-$ 3), 134.0 (C-2), 128.5 (C-12), 128.5 (C-13), 126.1 (C-14), 117.6 (C-5), 60.5 (C-7), 35.7 (C-9), 35.2 (C-10), 14.5 (C-8), 14.0 (C-1)

HRMS $\left(E S I^{+}\right)$calculated for $\mathrm{C}_{16} \mathrm{H}_{21} \mathrm{O}_{2}[\mathrm{M}+\mathrm{H}]^{+}=245.1537$; found $=245.1543$

IR (neat) $\vee\left(\mathrm{cm}^{-1}\right)=2932,1711,1630,1613,1273,1173,1039,980$

\section{$\underline{(+)-2-(2-([1,1 '-b i p h e n y l]-4-y l) c y c l o p e n t-2-e n-1-y l) \text { propan-2-ol } 8}$}

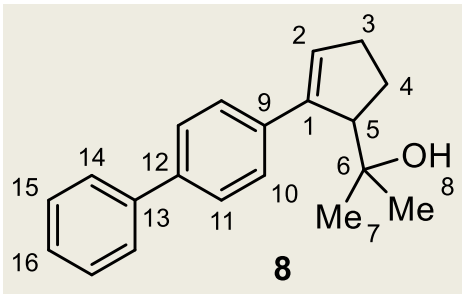

Synthesized following general procedure $\mathbf{A}$ as the sole product instead of dienoates starting from 7. Obtained as a light yellow solid after flash chromatography using pentane/Et $\mathrm{t}_{2} \mathrm{O} 85: 15$ as eluent $(60 \%$ yield, $93 \%$ ee). VCPs 7 were obtained from an enantioenriched sample of $\mathbf{3 a}$, which was prepared according to treated with methyl lithium (2 equivalents as a 1.6 M solution in diethyl ether), added dropwise at $0{ }^{\circ} \mathrm{C}$. After 1 hour of stirring at room temperature, the reaction was quenched by addition of a saturated $\mathrm{NH}_{4} \mathrm{Cl}$ solution at $0{ }^{\circ} \mathrm{C}$ and extracted 3 times with ethyl acetate. The collected 
organic phases were washed with a saturated $\mathrm{NaHCO}_{3}$ solution, dried over anhydrous $\mathrm{Na}_{2} \mathrm{SO}_{4}$ and evaporated at reduced pressure. After purification by flash chromatography using pentane/ethyl acetate $90: 10$ as eluent, 7 was obtained as a white solid $(41.8 \mathrm{mg}, 88 \%$ yield,

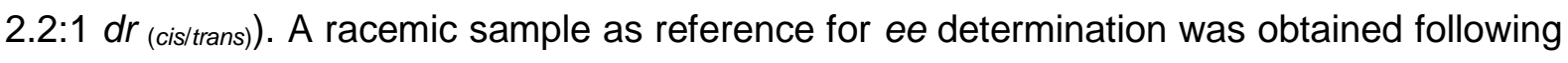
the same procedure with $\mathbf{L}_{\mathbf{1}}$ as achiral cyclopropanation ligand.

${ }^{1} \mathrm{H}$ NMR $\left(\mathrm{CDCl}_{3}, 400 \mathrm{MHz}\right) \delta(\mathrm{ppm})=7.64-7.47(\mathrm{~m}, 6 \mathrm{H}, \mathrm{H}-10+\mathrm{H}-11+\mathrm{H}-14), 7.47-7.39(\mathrm{~m}, 2 \mathrm{H}$, $\mathrm{H}-15), 7.37-7.30(\mathrm{~m}, 1 \mathrm{H}, \mathrm{H}-16), 6.16-6.04(\mathrm{~m}, 1 \mathrm{H}, \mathrm{H}-2), 3.39\left(\mathrm{~d},{ }^{3} \mathrm{~J}_{\mathrm{H}-\mathrm{H}}=9.4 \mathrm{~Hz}, 1 \mathrm{H}, \mathrm{H}-5\right), 2.58-$ 2.36 (m, 2H, H-3), 2.32-2.13 (m, 1H, H-4a), 2.09-1.95 (m, 1H, H-4b), 1.19 (s, 3H, H-7a), 1.12 (s, $1 \mathrm{H}, \mathrm{H}-7 \mathrm{~b})$

${ }^{13} \mathrm{C}\left\{{ }^{1} \mathrm{H}\right\}$ NMR $\left(\mathrm{CDCl}_{3}, 101 \mathrm{MHz}\right) \delta(\mathrm{ppm})=144.6(\mathrm{C}-1), 140.9(\mathrm{C}-13), 139.9(\mathrm{C}-12), 138.0(\mathrm{C}-$ 9), 132.1 (C-2), 128.9 (C-15), 127.4 (C-10/C-11/C-14), 127.4 (C-16), 127.2 (C-10/C-11/C-14), 127.1 (C-10/C-11/C-14), 75.2 (C-6), 56.7 (C-5), 32.4 (C-3), 28.4 (C-7a), 28.0 (C-4), 27.1 (C7b)

MP $\left({ }^{\circ} \mathrm{C}\right)=93-97$

HRMS $\left(\mathrm{ESI}^{+}\right)$calculated for $\mathrm{C}_{20} \mathrm{H}_{21}[\mathrm{M}-\mathrm{OH}]^{+}=261.1638$; found $=261.1650$

IR (neat) $v\left(\mathrm{~cm}^{-1}\right)=3374,2968,1487,1373,1154,1120,833$

$[\alpha]^{20}{ }_{D}\left(c=0.25, \mathrm{CHCl}_{3}\right)=+215.9$

HPLC (ADH column, hexane/i-PrOH 90:10, flow $0.5 \mathrm{ml} / \mathrm{min}, \lambda=218 \mathrm{~nm}) \mathrm{t}_{\mathrm{R}}(\mathrm{min})=14.9$ and 19.4

Ethyl trans-4-(4-isobutylphenyl)-3-methyl-2-phenyl-3,6-dihydro-2H-1,2-oxazine-6-carboxylate 10

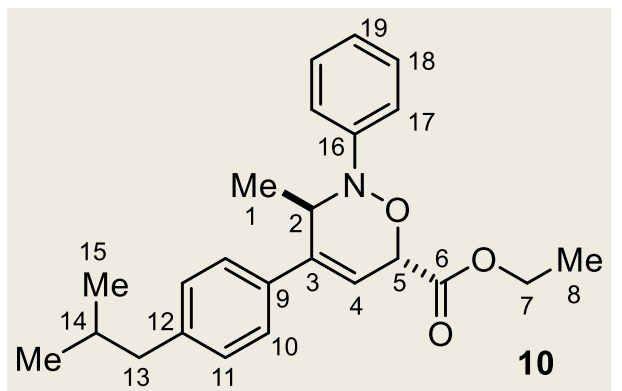

A sample of $5 \mathrm{c}(68.1 \mathrm{mg}, 0.25 \mathrm{mmol})$ was dissolved in $2.5 \mathrm{ml}$ of tetrahydrofuran. Nitrosobenzene $(53.6 \mathrm{mg}, 0.5$ $\mathrm{mmol}$ ) was added and the reaction mixture was stirred at $60^{\circ} \mathrm{C}$ for 18 hours. After concentration under reduced pressure the residue was purified by flash chromatography using pentane/Et ${ }_{2} \mathrm{O} 96: 4$ as eluent. Obtained as white solid (73 $\mathrm{mg}, 77 \%$ yield).

${ }^{1} \mathrm{H} \mathrm{NMR}\left(\mathrm{CDCl}_{3}, 400 \mathrm{MHz}\right) \delta(\mathrm{ppm})=7.38-7.26(\mathrm{~m}, 6 \mathrm{H}, \mathrm{H}-10+\mathrm{H}-17+\mathrm{H} 18), 7.17\left(\mathrm{~d},{ }^{3} \mathrm{~J}_{\mathrm{H}-\mathrm{H}}=8.2\right.$ $\mathrm{Hz}, 2 \mathrm{H}, \mathrm{H}-11), 6.97\left(\mathrm{tt},{ }^{3} \mathrm{~J}_{\mathrm{H}-\mathrm{H}}=7.1 \mathrm{~Hz},{ }^{4} \mathrm{~J}_{\mathrm{H}-\mathrm{H}}=1.3 \mathrm{~Hz}, 1 \mathrm{H}, \mathrm{H}-19\right), 6.32\left(\mathrm{dd},{ }^{3} \mathrm{~J}_{\mathrm{H}-\mathrm{H}}=4.4 \mathrm{~Hz},{ }^{4} \mathrm{~J}_{\mathrm{H}-\mathrm{H}}\right.$ $=0.7 \mathrm{~Hz}, 1 \mathrm{H}, \mathrm{H}-4), 5.03\left(\mathrm{~d},{ }^{3} \mathrm{~J}_{\mathrm{H}-\mathrm{H}}=4.4 \mathrm{~Hz}, 1 \mathrm{H}, \mathrm{H}-5\right), 4.71\left(\mathrm{q},{ }^{3} \mathrm{~J}_{\mathrm{H}-\mathrm{H}}=6.5 \mathrm{~Hz}, 1 \mathrm{H}, \mathrm{H}-2\right), 4.26-$ 
$4.11(\mathrm{~m}, 2 \mathrm{H}, \mathrm{H}-7), 2.50\left(\mathrm{~d},{ }^{3} \mathrm{~J}_{\mathrm{H}-\mathrm{H}}=7.2 \mathrm{~Hz}, 2 \mathrm{H}, \mathrm{H}-13\right), 1.95-1.82(\mathrm{~m}, 1 \mathrm{H}, \mathrm{H}-14), 1.22\left(\mathrm{t},{ }^{3} \mathrm{~J}_{\mathrm{H}-\mathrm{H}}=\right.$ $7.1 \mathrm{~Hz}, 3 \mathrm{H}, \mathrm{H}-8), 1.14\left(\mathrm{~d},{ }^{3} \mathrm{~J}_{\mathrm{H}-\mathrm{H}}=6.5 \mathrm{~Hz}, 3 \mathrm{H}, \mathrm{H}-1\right), 0.95-0.90(\mathrm{~m}, 6 \mathrm{H}, \mathrm{H}-15)$

${ }^{13} \mathrm{C}\left\{{ }^{1} \mathrm{H}\right\}$ NMR $\left(\mathrm{CDCl}_{3}, 101 \mathrm{MHz}\right) \delta(\mathrm{ppm})=170.2(\mathrm{C}-6), 148.1(\mathrm{C}-16), 142.3(\mathrm{C}-3), 142.1(\mathrm{C}-$ 12), 135.0 (C-9), 129.6 (C-11), 128.8 (C-18), 126.0 (C-10), 121.5 (C-19), 118.7 (C-4), 115.9 (C-17), 76.7 (C-5), 61.4 (C-7), 55.4 (C-2), 45.2 (C-13), 30.4 (C-14), 22.5 (C-15), 14.3 (C-8), $12.1(\mathrm{C}-1)$

MP $\left({ }^{\circ} \mathrm{C}\right)=96-100$

HRMS $\left(\mathrm{ESI}^{+}\right)$calculated for $\mathrm{C}_{24} \mathrm{H}_{29} \mathrm{NO}_{3}[\mathrm{M}+\mathrm{H}]^{+}=380.2226$; found $=380.2223$

IR $($ neat $) \vee\left(\mathrm{cm}^{-1}\right)=2961,1731,1597,1492,1216,1028$ 


\section{Ru-catalyzed isomerization of VPCs to cross-conjugated dienoates}

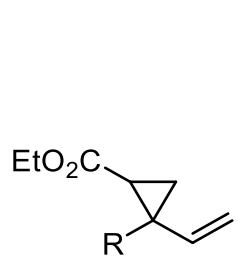

cis/trans-3a-h

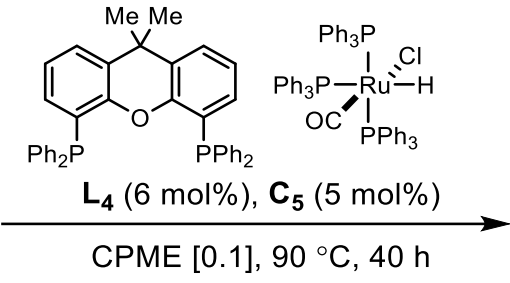

CPME [0.1], $90^{\circ} \mathrm{C}, 40 \mathrm{~h}$

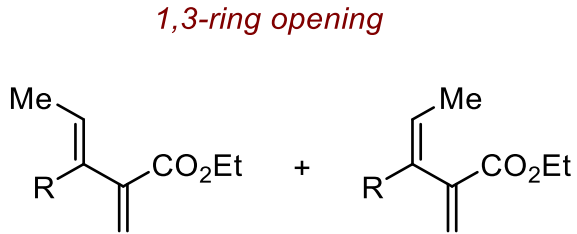

(E)-6a-h

(Z)-6a-h

FigureS4. Ru-catalyzed isomerization of VCPs to conjugated dienoates.

\section{General procedure - B}

In a $5 \mathrm{~mL}$ flame-dried Schlenk tube, the mixture of vinylcyclopropanes $\mathbf{3 a}-\mathrm{h}(0.25 \mathrm{mmol})$, $\left[\mathrm{RuHCl}(\mathrm{CO})\left(\mathrm{PPh}_{3}\right)_{3}\right] \mathbf{C}_{5}(0.0125 \mathrm{mmol})$ and Xanthphos $\mathbf{L}_{4}(0.15 \mathrm{mmol})$ were dissolved in 2.5 $\mathrm{mL}$ of anhydrous and degassed cyclopentyl methyl ether. The reaction mixture was stirred at $90{ }^{\circ} \mathrm{C}$ for 40 hours.

\subsection{Reaction optimization}

Catalysts and reaction conditions were tested according to general procedure $\mathbf{B}$ using the mixture of $3 \mathbf{a}$ as model substrate $\left(\right.$ cis/trans $\left.=1.7: 1, r_{(3 a / 4 a)}=13.3: 1\right)$.

\begin{tabular}{cccccc}
\hline Precatalyst & Solvent & $\mathbf{T}\left({ }^{\circ} \mathbf{C}\right)$ & $\mathbf{t}(\mathbf{h})$ & ${\text { Conv. }(\%)^{a}}^{a}$ & $\mathbf{E} / \mathbf{Z}$ \\
\hline$\left[\mathrm{Ru}(\mathrm{H}) \mathrm{Cl}\left(\mathrm{PPh}_{3}\right)_{3}\right] \mathbf{C}_{4}$ & Toluene & 80 & 20 & 6 & n.d. ${ }^{b}$ \\
{$\left[\mathrm{Ru}(\mathrm{H}) \mathrm{Cl}(\mathrm{CO})\left(\mathrm{PPh}_{3}\right)_{3}\right] \mathbf{C}_{5}$} & Toluene & 60 & 20 & 54 & $2.6: 1$ \\
{$\left[\mathrm{Ru}(\mathrm{H})_{2}(\mathrm{CO})\left(\mathrm{PPh}_{3}\right)_{3}\right] \mathbf{C}_{7}$} & Toluene & 80 & 20 & 48 & $2.2: 1$ \\
{$\left[\mathrm{Rh}(\mathrm{H})(\mathrm{CO})\left(\mathrm{PPh}_{3}\right)_{3}\right] \mathbf{C}_{8}$} & Toluene & 80 & 20 & n.r. ${ }^{c}$ & - \\
{$\left[\mathrm{RuHCl}(\mathrm{CO})\left(\mathrm{PPh}_{3}\right)_{3}\right] \mathbf{C}_{5}$} & $1,2-\mathrm{DCE}$ & 60 & 40 & 46 & $2.3: 1$ \\
{$\left[\mathrm{RuHCl}(\mathrm{CO})\left(\mathrm{PPh}_{3}\right)_{3}\right] \mathbf{C}_{5}$} & THF & 60 & 40 & 63 & $2.8: 1$ \\
{$\left[\mathrm{RuHCl}(\mathrm{CO})\left(\mathrm{PPh}_{3}\right)_{3}\right] \mathbf{C}_{5}$} & $\mathrm{CPME}$ & 60 & 40 & 80 & $2.9: 1$ \\
{$\left[\mathrm{RuHCl}(\mathrm{CO})\left(\mathrm{PPh}_{3}\right)_{3}\right] \mathbf{C}_{5}$} & $\mathrm{MTBE}$ & 60 & 40 & 41 & $2.3: 1$ \\
{$\left[\mathrm{RuHCl}(\mathrm{CO})\left(\mathrm{PPh}_{3}\right)_{3}\right] \mathbf{C}_{5}$} & 2-Me-THF & 60 & 40 & 69 & $2.9: 1$ \\
\hline
\end{tabular}

Table S2. Screening of precatalysts and solvents for Ru-catalyzed ring opening. ${ }^{a}$ Determined by ${ }^{1} \mathrm{H}$ NMR using mesitylene as internal standard. ${ }^{b}$ Not determined. ${ }^{c}$ No reaction. 
The effect of different ligands $(6 \mathrm{~mol} \%)$ and reaction temperatures was tested according to general procedure $\mathbf{B}$ using the mixture of $\mathbf{3 a}$ as model substrate.

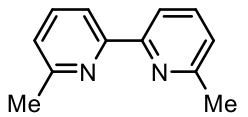

$\mathrm{L}_{1}\left(6,6^{\prime}-\mathrm{Me}_{2}\right.$-bipy $)$

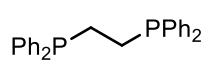

$\mathrm{L}_{2}$ (dppe)

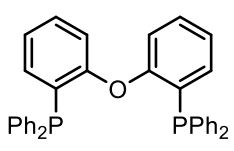

$\mathrm{L}_{3}$ (DPE-phos)

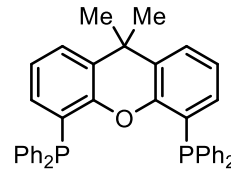

$\mathrm{L}_{4}$ (xantphos)

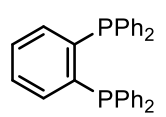

$\mathrm{L}_{5}$ (dppbz)

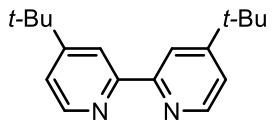

$\mathrm{L}_{6}\left(4,4^{\prime}-\mathrm{Me}_{2}\right.$-bipy $)$

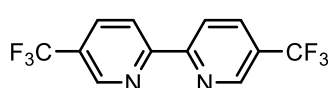

$\mathrm{L}_{7}\left(3,3^{\prime}-\left(\mathrm{CF}_{3}\right)_{2}\right.$-bipy $)$

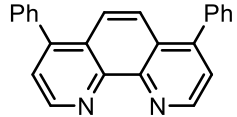

$\mathrm{L}_{8}$ (bathophenanthrolin)

\begin{tabular}{ccccc}
\hline Ligand & $\mathbf{T}\left({ }^{\circ} \mathbf{C}\right)$ & $\mathbf{t}(\mathbf{h})$ & Conv. $(\%)^{a}$ & $\mathbf{E}: \mathbf{Z}$ \\
\hline- & 60 & 40 & 80 & $2.9: 1$ \\
$\mathrm{PCy}{ }^{b}$ & 60 & 40 & n.r. & - \\
$\mathrm{PPh}_{3}{ }^{c}$ & 60 & 40 & 48 & $2.0: 1$ \\
$\mathbf{L}$ & 60 & 40 & 64 & $2.8: 1$ \\
$\mathbf{L}_{2}$ & 60 & 40 & 21 & $2.1: 1$ \\
$\mathbf{L}_{3}$ & 60 & 40 & 25 & $2.0: 1$ \\
$\mathbf{L}_{4}$ & 60 & 40 & 50 & $4.5: 1$ \\
$\mathbf{L}_{5}$ & 60 & 40 & 8 & $1: 1$ \\
$\mathbf{L}_{6}$ & 60 & 40 & 74 & $2.8: 1$ \\
$\mathbf{L}_{\mathbf{7}}$ & 60 & 40 & 50 & $2.4: 1$ \\
$\mathbf{L}_{8}$ & 60 & 40 & n.r. & \\
$\mathbf{L}_{4}$ & 40 & 88 & 24 & $7.0: 1$ \\
$\mathbf{L}_{4}$ & 90 & 40 & $72(57)^{e}$ & $4.2: 1$ \\
$\mathbf{L}_{4}$ & 90 & 64 & 74 & $3.9: 1$ \\
\hline
\end{tabular}

Table S3. Screening of ligands and reaction temperatures for Ru-catalyzed ring opening. ${ }^{a}$ Determined by ${ }^{1} \mathrm{H}$ NMR using mesitylene as internal standard. ${ }^{b} 15 \mathrm{~mol} \% .{ }^{c} 5 \mathrm{~mol} \% .{ }^{d}$ No reaction. ${ }^{e}$ Isolated yield. 


\subsection{Reaction scope}

The VCPs obtained by diene cyclopropanation were subjected to general procedure $\mathbf{B}$. Results are reported in FigureS5.

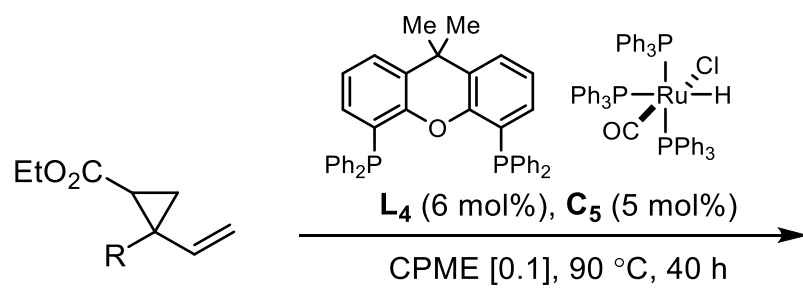

cis/trans-3a-h<smiles>C=C(C(=O)OCC)C(=O)OCC</smiles>

$(E) /(Z) 4.2: 1$<smiles>C=C(OCC)C(=O)OCC</smiles>

$(E) /(Z) 2.6: 1$<smiles>C=C(C(=O)OCC)/C(=C/C)c1ccc(OC)cc1</smiles>

$(E) /(Z) 4.8: 1$<smiles>CC=C(C(=O)OCC)C(=CC(C)(C)C)c1ccc(C(C)(C)C)cc1</smiles>

$(E) /(Z) 4.5: 1$

(E)-6a-h (Z)-6a-h

(E)-6a-h (Z)-6a-h

B. 1,3-ring opening<smiles>[R]C(=CC)C(=C)C(=O)OCC</smiles><smiles>C=C(C(=O)OCC)C(=CC)c1ccsc1</smiles>

$(E) /(Z) 4.7: 1$<smiles>C=C(C(=O)OCC)/C(=C/C)c1ccc2ccccc2c1</smiles>

$(E) /(Z) 4.7: 1$<smiles>C=C(C(=O)OCC)/C(=C/C)c1cn(C(=O)OC(C)(C)C)c2ccccc12</smiles><smiles>C=C(C(=O)OCC)C(=CC)CCc1ccccc1</smiles>

$(E) /(Z) 1: 1$

FigureS5. Scope of the Ru-catalyzed ring opening. Selectivity determined by ${ }^{1} \mathrm{H}$ NMR. Yield after purification. ${ }^{a}$ Product dienoates were not successfully separated from unreacted VCPs $\mathbf{3}$ and $\mathbf{4}$, conversion is reported in the place of yield. 


\subsection{Data of new compounds}

Ethyl $(E)-3-\left(\left[1,1^{\prime}-\right.\right.$ biphenyl]-4-yl)-2-methylenepent-3-enoate $(E)-6 a$

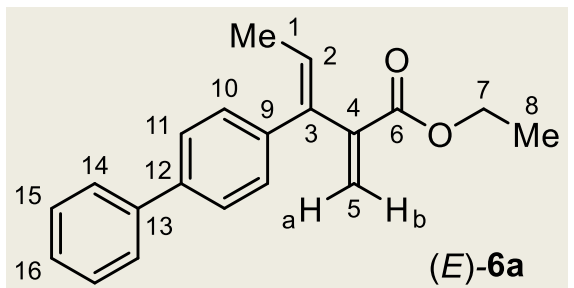

Synthesized according to general procedure B starting from a racemic mixture of $\mathbf{3 a}$ and $\mathbf{4 a}$ isomers. Obtained as a colorless oil after flash chromatography using pentane/ $\mathrm{Et}_{2} \mathrm{O} 97: 3$ as eluent $(41.7 \mathrm{mg}, 57 \%$ yield, $5.6: 1$

$(E) /(Z)(4.2: 1$ in the crude), $92 \%$ purity). The VCP mixture was obtained according to the general cyclopropanation procedure (13.3:1 $r r_{(3 a / 4 a)}, 1.7: 1 d r$ (cis/trans)).

${ }^{1} \mathrm{H}$ NMR $\left(\mathrm{CDCl}_{3}, 400 \mathrm{MHz}\right) \delta(\mathrm{ppm})=7.64-7.60(\mathrm{~m}, 2 \mathrm{H}, \mathrm{H}-14), 7.60-7.56(\mathrm{~m}, 2 \mathrm{H}, \mathrm{H}-11), 7.47-$ $7.41(\mathrm{~m}, 2 \mathrm{H}, \mathrm{H}-15), 7.37-7.32(\mathrm{~m}, 1 \mathrm{H}, \mathrm{H}-16), 7.28-7.23(\mathrm{~m}, 2 \mathrm{H}, \mathrm{H}-10), 6.10\left(\mathrm{q},{ }^{3} \mathrm{~J}_{\mathrm{H}-\mathrm{H}}=7.1 \mathrm{~Hz}\right.$, $1 \mathrm{H}, \mathrm{H}-2), 6.01\left(\mathrm{~d},{ }^{2} \mathrm{~J}_{\mathrm{H}-\mathrm{H}}=1.6,1 \mathrm{H}, \mathrm{H}-5 \mathrm{~b}\right), 5.52\left(\mathrm{~d},{ }^{2} \mathrm{~J}_{\mathrm{H}-\mathrm{H}}=1.6 \mathrm{~Hz}, 1 \mathrm{H}, \mathrm{H}-5 \mathrm{a}\right), 4.13\left(\mathrm{q},{ }^{3} \mathrm{~J}_{\mathrm{H}-\mathrm{H}}=7.1\right.$ $\mathrm{Hz}, 2 \mathrm{H}, \mathrm{H}-7), 1.77\left(\mathrm{~d},{ }^{3} \mathrm{~J}_{\mathrm{H}-\mathrm{H}}=7.1 \mathrm{~Hz}, 3 \mathrm{H}, \mathrm{H}-1\right), 1.16\left(\mathrm{t},{ }^{3} \mathrm{~J}_{\mathrm{H}-\mathrm{H}}=7.1 \mathrm{~Hz}, 3 \mathrm{H}, \mathrm{H}-8\right)$

${ }^{13} \mathrm{C}\left\{{ }^{1} \mathrm{H}\right\}$ NMR $\left(\mathrm{CDCl}_{3}, 101 \mathrm{MHz}\right) \delta(\mathrm{ppm})=167.6(\mathrm{C}-6), 144.1(\mathrm{C}-4), 141.0(\mathrm{C}-13), 139.8(\mathrm{C}-$ 12), 138.8 (C-3), 137.5 (C-9), 129.8 (C-10), 128.9 (C-15), 127.6 (C-2), 127.4 (C-16), 127.2 (C14), 126.9 (C-11), 124.5 (C-5), 60.9 (C-7), 15.5 (C-1), 14.1 (C-8)

HRMS $\left(\mathrm{ESI}^{+}\right)$calculated for $\mathrm{C}_{20} \mathrm{H}_{21} \mathrm{O}_{2}[\mathrm{M}+\mathrm{H}]^{+}=293.1536$; found $=293.1520$

IR (neat) $\vee\left(\mathrm{cm}^{-1}\right)=2919,1718,1487,1214,1143,842$

\section{Ethyl (Z)-3-([1,1'-biphenyl]-4-yl)-2-methylenepent-3-enoate (Z)-6a}

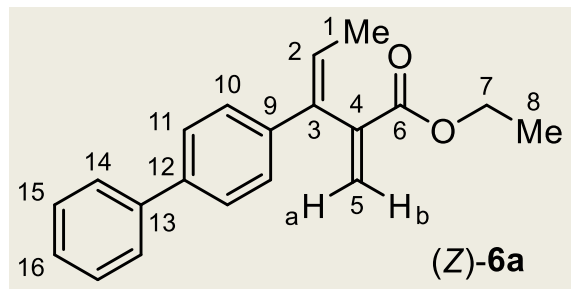

Obtained as minor product from the synthesis of isomer $(E)-6 a(57 \%$ yield, $5.6: 1(E) /(Z)(4.2: 1$ in the crude $))$.

${ }^{1} \mathrm{H}$ NMR $\left(\mathrm{CDCl}_{3}, 400 \mathrm{MHz}\right) \delta(\mathrm{ppm})=7.44-7.60(\mathrm{~m}, 2 \mathrm{H}$, $\mathrm{H}-14)$, 7.54-7.50 (m, 2H, H-11), 7.47-7.41 (m, 2H, H-15), 7.40-7.36 (m, 2H, H-10), 7.36-7.32 (m, 1H, H-16), 6.63 (d, $\left.{ }^{2} \mathrm{~J}_{\mathrm{H}-\mathrm{H}}=1.8 \mathrm{~Hz}, 1 \mathrm{H}, \mathrm{H}-5 \mathrm{~b}\right), 6.21\left(\mathrm{q},{ }^{3} \mathrm{~J}_{\mathrm{H}-\mathrm{H}}=7.0 \mathrm{~Hz}, 1 \mathrm{H}, \mathrm{H}-2\right), 5.72\left(\mathrm{~d},{ }^{2} \mathrm{~J}_{\mathrm{H}-\mathrm{H}}=1.9 \mathrm{~Hz}, 1 \mathrm{H}, \mathrm{H}-5 \mathrm{a}\right)$, $4.14\left(\mathrm{q},{ }^{3} \mathrm{~J}_{\mathrm{H}-\mathrm{H}}=7.1,2 \mathrm{H}, \mathrm{H}-7\right), 1.77\left(\mathrm{~d},{ }^{3} \mathrm{~J}_{\mathrm{H}-\mathrm{H}}=7.1 \mathrm{~Hz}, 3 \mathrm{H}, \mathrm{H}-1\right), 14.2\left(\mathrm{t},{ }^{3} \mathrm{~J}_{\mathrm{H}-\mathrm{H}}=7.1 \mathrm{~Hz}, 3 \mathrm{H}, \mathrm{H}-\right.$ 8)

${ }^{13} \mathrm{C}\left\{{ }^{1} \mathrm{H}\right\}$ NMR $\left(\mathrm{CDCl}_{3}, 101 \mathrm{MHz}\right) \delta(\mathrm{ppm})=166.7$ (C-6), 141.0 (C-12/C-13), 140.8 (C-12/C-13), 140.1 (C-4), 139.0 (C-9), 138.1 (C-3), 129.7 (C-5), 128.9 (C-15), 127.3 (C-16), 127.1 (C-14+C11), 126.5 (C-10), 126.3 (C-2), 61.0 (C-7), 15.8 (C-1), 14.2 (C-8) 
Ethyl (E)-3-(4-methoxyphenyl)-2-methylenepent-3-enoate $(E)-6 \mathbf{b}$

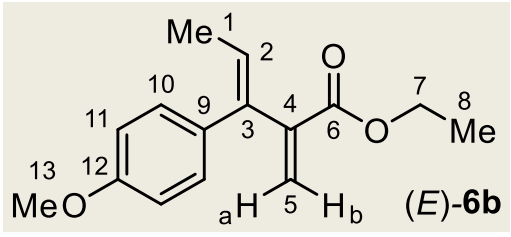

Synthesized according to general procedure B starting from a racemic mixture of $\mathbf{3} \mathbf{b}$ and $\mathbf{4 b}$ isomers. Obtained as $\mathbf{a}$ colorless oil after flash chromatography using pentane/Et $\mathrm{t}_{2} \mathrm{O}$ 97.5:2.5 as eluent (40.6 mg, 66\% yield, 4.8:1 (E)/(Z), 98\% purity). The VCP mixture was obtained according to the general cyclopropanation procedure $\left(14.3: 1 r_{(3 b / 4 b)}, 1.2: 1 d r_{(\text {cis/trans })}\right)$.

${ }^{1} \mathrm{H}$ NMR $\left(\mathrm{CDCl}_{3}, 400 \mathrm{MHz}\right) \delta(\mathrm{ppm})=7.13-7.07(\mathrm{~m}, 2 \mathrm{H}, \mathrm{H}-10), 6.90-6.85(\mathrm{~m}, 2 \mathrm{H}, \mathrm{H}-11), 6.01$ (q, $\left.{ }^{3} \mathrm{~J}_{\mathrm{H}-\mathrm{H}}=7.1 \mathrm{~Hz}, 1 \mathrm{H}, \mathrm{H}-2\right), 5.93\left(\mathrm{~d},{ }^{2} \mathrm{~J}_{\mathrm{H}-\mathrm{H}}=1.6 \mathrm{~Hz}, 1 \mathrm{H}, \mathrm{H}-5 \mathrm{~b}\right), 5.44\left(\mathrm{~d},{ }^{2} \mathrm{~J}_{\mathrm{H}-\mathrm{H}}=1.6 \mathrm{~Hz}, 1 \mathrm{H}, \mathrm{H}-\right.$ 5a), $4.12\left(\mathrm{q},{ }^{3} \mathrm{~J}_{\mathrm{H}-\mathrm{H}}=7.1 \mathrm{~Hz}, 2 \mathrm{H}, \mathrm{H}-7\right), 3.81(\mathrm{~s}, 3 \mathrm{H}, \mathrm{H}-13), 1.71\left(\mathrm{~d},{ }^{3} \mathrm{~J}_{\mathrm{H}-\mathrm{H}}=7.1 \mathrm{~Hz}, 3 \mathrm{H}, \mathrm{H}-1\right), 1.16$ $\left(\mathrm{t},{ }^{3} \mathrm{~J}_{\mathrm{H}-\mathrm{H}}=7.1 \mathrm{~Hz}, 3 \mathrm{H}, \mathrm{H}-8\right)$

${ }^{13} \mathrm{C}\left\{{ }^{1} \mathrm{H}\right\}$ NMR $\left(\mathrm{CDCl}_{3}, 101 \mathrm{MHz}\right) \delta(\mathrm{ppm})=167.8(\mathrm{C}-6), 158.6(\mathrm{C}-12), 144.4(\mathrm{C}-4), 138.7(\mathrm{C}-$ 3), 130.7 (C-9), 130.5 (C-10), 127.0 (C-2), 123.9 (C-5), 113.6 (C-11), 60.8 (C-7), 55.3 (C-13), $15.4(\mathrm{C}-1), 14.2(\mathrm{C}-8)$

HRMS $\left(\mathrm{ESI}^{+}\right)$calculated for $\mathrm{C}_{15} \mathrm{H}_{19} \mathrm{O}_{3}[\mathrm{M}+\mathrm{H}]^{+}=247.1329 ;$ found $=247.1344$

IR $($ neat $) \vee\left(\mathrm{cm}^{-1}\right)=1608,1510,1245,1175,1032$

Ethyl (Z)-3-(4-methoxyphenyl)-2-methylenepent-3-enoate (Z)-6b

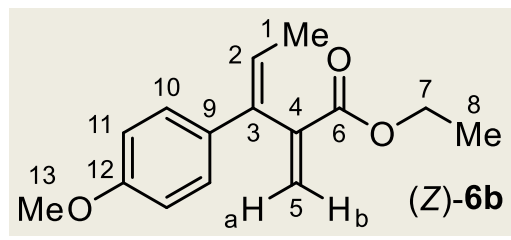

Obtained as minor product from the synthesis of $(E)-6 \mathbf{b}(66 \%$ yield, 4.8:1 $(E) /(Z))$.

${ }^{1} \mathrm{H} \mathrm{NMR}\left(\mathrm{CDCl}_{3}, 400 \mathrm{MHz}\right) \delta(\mathrm{ppm})=7.25-7.20(\mathrm{~m}, 2 \mathrm{H}, \mathrm{H}-10)$, 6.84-6.79 (m, 2H, H-11), $6.57\left(\mathrm{~d},{ }^{2} \mathrm{~J}_{\mathrm{H}-\mathrm{H}}=1.9 \mathrm{~Hz}, 1 \mathrm{H}, \mathrm{H}-5 \mathrm{~b}\right)$, $6.03\left(\mathrm{q},{ }^{3} \mathrm{~J}_{\mathrm{H}-\mathrm{H}}=7.0 \mathrm{~Hz}, 1 \mathrm{H}, \mathrm{H}-2\right), 5.65\left(\mathrm{~d},{ }^{2} \mathrm{~J}_{\mathrm{H}-\mathrm{H}}=1.9 \mathrm{~Hz}, 1 \mathrm{H}, \mathrm{H}-5 \mathrm{a}\right), 4.12\left(\mathrm{q},{ }^{3} \mathrm{~J}_{\mathrm{H}-\mathrm{H}}=7.1 \mathrm{~Hz}, 2 \mathrm{H}\right.$, $\mathrm{H}-7), 3.79(\mathrm{~s}, 3 \mathrm{H}, \mathrm{H}-13), 1.78\left(\mathrm{~d},{ }^{3} \mathrm{~J}_{\mathrm{H}-\mathrm{H}}=7.0 \mathrm{~Hz}, 3 \mathrm{H}, \mathrm{H}-1\right), 1.14\left(\mathrm{t},{ }^{3} \mathrm{~J}_{\mathrm{H}-\mathrm{H}}=7.1 \mathrm{~Hz}, 3 \mathrm{H}, \mathrm{H}-8\right)$

${ }^{13} \mathrm{C}\left\{{ }^{1} \mathrm{H}\right\}$ NMR $\left(\mathrm{CDCl}_{3}, 101 \mathrm{MHz}\right) \delta(\mathrm{ppm})=166.8(\mathrm{C}-6), 158.9(\mathrm{C}-12), 139.2(\mathrm{C}-4), 137.9(\mathrm{C}-$ 3), 133.9 (C-9), 129.3 (C-5), 127.3 (C-10), 124.6 (C-2), 113.8 (C-11), 60.9 (C-7), 55.4 (C-13), $15.7(\mathrm{C}-1), 14.2(\mathrm{C}-8)$ 


\section{Ethyl (E)-3-(4-isobutylphenyl)-2-methylenepent-3-enoate $(E)-6 c$}

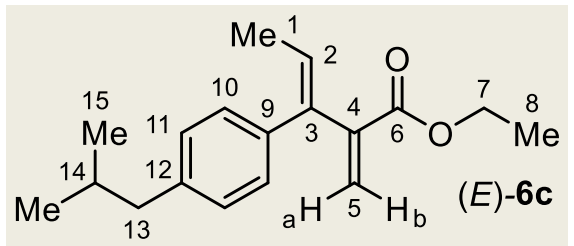

Synthesized according to general procedure B starting from a racemic mixture of $\mathbf{3 c}$ and $\mathbf{4 c}$ isomers. Obtained as a colorless oil after flash chromatography using pentane/Et $\mathrm{E}_{2} \mathrm{O} 98.5: 1.5$ as eluent $(51.7 \mathrm{mg}, 76 \%$ yield, 4.5:1 $(E) /(Z), 98 \%$ purity). The VCP mixture was obtained according to the general

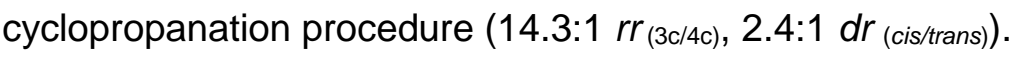

$4 \mathrm{mmol}$ scale reaction: $0.716 \mathrm{mg}, 65 \%$ yield, $4.8: 1(E) /(Z)$

${ }^{1} \mathrm{H}$ NMR $\left(\mathrm{CDCl}_{3}, 400 \mathrm{MHz}\right) \delta(\mathrm{ppm})=7.13-7.03(\mathrm{~m}, 4 \mathrm{H}, \mathrm{H}-10+\mathrm{H}-11), 6.04\left(\mathrm{q},{ }^{3} \mathrm{~J}_{\mathrm{H}-\mathrm{H}}=7.1 \mathrm{~Hz}\right.$, $1 \mathrm{H}, \mathrm{H}-2), 5.94\left(\mathrm{~d},{ }^{2} \mathrm{~J}_{\mathrm{H}-\mathrm{H}}=1.7 \mathrm{~Hz}, 1 \mathrm{H}, \mathrm{H}-5 \mathrm{~b}\right), 5.44\left(\mathrm{~d},{ }^{2} \mathrm{~J}_{\mathrm{H}-\mathrm{H}}=1.6 \mathrm{~Hz}, 1 \mathrm{H}, \mathrm{H}-5 \mathrm{a}\right), 4.11\left(\mathrm{q},{ }^{3} \mathrm{~J}_{\mathrm{H}-\mathrm{H}}=\right.$ $7.1 \mathrm{~Hz}, 2 \mathrm{H}, \mathrm{H}-7), 2.47\left(\mathrm{~d},{ }^{3} \mathrm{~J}_{\mathrm{H}-\mathrm{H}}=7.2 \mathrm{~Hz}, 2 \mathrm{H}, \mathrm{H}-13\right), 1.92-1.81(\mathrm{~m}, 1 \mathrm{H}, \mathrm{H}-14), 1.71\left(\mathrm{~d},{ }^{3} \mathrm{~J}_{\mathrm{H}-\mathrm{H}}=\right.$ $7.1 \mathrm{~Hz}, 3 \mathrm{H}, \mathrm{H}-1), 1.13\left(\mathrm{t}, 3^{3} \mathrm{H}_{\mathrm{H}-\mathrm{H}}=7.1 \mathrm{~Hz}, 3 \mathrm{H}, \mathrm{H}-8\right), 0.90\left(\mathrm{~d},{ }^{3} \mathrm{~J}_{\mathrm{H}-\mathrm{H}}=6.6 \mathrm{~Hz}, 6 \mathrm{H}, \mathrm{H}-15\right)$

${ }^{13} \mathrm{C}\left\{{ }^{1} \mathrm{H}\right\}$ NMR $\left(\mathrm{CDCl}_{3}, 101 \mathrm{MHz}\right) \delta(\mathrm{ppm})=167.8(\mathrm{C}-6), 144.4(\mathrm{C}-4), 140.5(\mathrm{C}-12), 139.1$ (C3), 135.6 (C-9), 129.1 (C-10/C-11), 128.9 (C-10/C-11), 127.0 (C-2), 124.0 (C-5), 60.8 (C-7), 45.3 (C-13), 30.4 (C-14), 22.5 (C-15), 15.5 (C-1), 14.1 (C-8)

HRMS $\left(\mathrm{ESI}^{+}\right)$calculated for $\mathrm{C}_{18} \mathrm{H}_{25} \mathrm{O}_{2}[\mathrm{M}+\mathrm{H}]^{+}=273.1850$; found $=273.1840$

IR (neat) $\vee\left(\mathrm{cm}^{-1}\right)=2956,1721,1216,1141,1024$

Ethyl (Z)-3-(4-isobutylphenyl)-2-methylenepent-3-enoate (Z)-6c

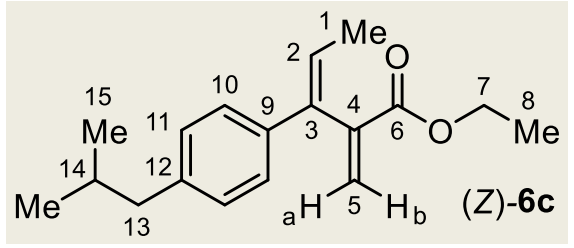

Obtained as minor product from the synthesis of $(E)-6 c$ $(76 \%$ yield, $4.5: 1(E) /(Z))$.

${ }^{1} \mathrm{H}$ NMR $\left(\mathrm{CDCl}_{3}, 400 \mathrm{MHz}\right) \delta(\mathrm{ppm})=7.23-7.18(\mathrm{~m}, 2 \mathrm{H}, \mathrm{H}-$ 10), 7.13-7.03 (m, 2H, H-11), $6.58\left(\mathrm{~d},{ }^{2} \mathrm{~J}_{\mathrm{H}-\mathrm{H}}=2.0 \mathrm{~Hz}, 1 \mathrm{H}\right.$, $\mathrm{H}-5 \mathrm{~b}), 6.11\left(\mathrm{q},{ }^{3} \mathrm{~J}_{\mathrm{H}-\mathrm{H}}=7.0 \mathrm{~Hz}, 1 \mathrm{H}, \mathrm{H}-2\right), 5.66\left(\mathrm{~d},{ }^{2} \mathrm{~J}_{\mathrm{H}-\mathrm{H}}=2.0 \mathrm{~Hz}, 1 \mathrm{H}, \mathrm{H}-5 \mathrm{a}\right), 4.11\left(\mathrm{q},{ }^{3} \mathrm{~J}_{\mathrm{H}-\mathrm{H}}=7.1\right.$ $\mathrm{Hz}, 2 \mathrm{H}, \mathrm{H}-7), 2.44\left(\mathrm{~d},{ }^{3} \mathrm{~J}_{\mathrm{H}-\mathrm{H}}=7.2 \mathrm{~Hz}, 2 \mathrm{H}, \mathrm{H}-13\right), 1.90-1.81(\mathrm{~m}, 1 \mathrm{H}, \mathrm{H}-14), 1.80\left(\mathrm{~d},{ }^{3} \mathrm{~J}_{\mathrm{H}-\mathrm{H}}=7.0\right.$ $\mathrm{Hz}, 3 \mathrm{H}, \mathrm{H}-1), 1.11\left(\mathrm{t},{ }^{3} \mathrm{~J}_{\mathrm{H}-\mathrm{H}}=7.1 \mathrm{~Hz}, 3 \mathrm{H}, \mathrm{H}-8\right), 0.91\left(\mathrm{~d},{ }^{3} \mathrm{~J}_{\mathrm{H}-\mathrm{H}}=6.6 \mathrm{~Hz}, 6 \mathrm{H}, \mathrm{H}-15\right)$

${ }^{13} \mathrm{C}\left\{{ }^{1} \mathrm{H}\right\}$ NMR $\left(\mathrm{CDCl}_{3}, 101 \mathrm{MHz}\right) \delta(\mathrm{ppm})=166.8(\mathrm{C}-6), 140.6(\mathrm{C}-12), 139.2(\mathrm{C}-4), 138.5(\mathrm{C}-$ 9), 138.3 (C-3), 129.4 (C-5/C-11), 129.2 (C-5/C-11), 125.8 (C-10), 125.3 (C-2), 60.8 (C-7), 45.2 (C-13), 30.3 (C-14), 22.5 (C-15), 15.7 (C-1), 14.1 (C-8) 
Ethyl (E)-2-methylene-3-(4-(trifluoromethyl)phenyl)pent-3-enoate $(E)$-6d

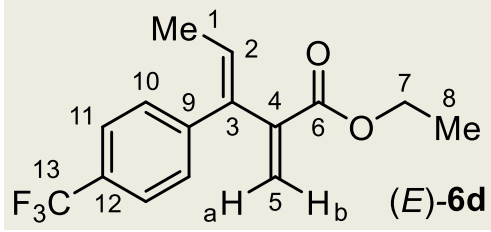

Synthesized according to general procedure $\mathbf{B}$ starting from a racemic mixture of $\mathbf{3} \mathbf{d}$ and $\mathbf{4} \mathbf{d}$ isomers (67\% conversion). Obtained as a yellowish oil after flash chromatography using pentane/Et ${ }_{2} \mathrm{O} 99: 1$ as eluent $(1.6: 1(E) /(Z)(2.6: 1$ in the crude), contains $28 \%$ of trans-4d). The VCP mixture was obtained according to the general cyclopropanation procedure $\left(4.4: 1 \mathrm{rr}_{(3 \mathrm{~d} / 4 \mathrm{~d})}, 1.7: 1 \mathrm{dr}\right.$ (cis/trans) $)$.

${ }^{1} \mathrm{H}$ NMR $\left(\mathrm{CDCl}_{3}, 400 \mathrm{MHz}\right) \delta(\mathrm{ppm})=7.60\left(\mathrm{~d},{ }^{3} \mathrm{~J}_{\mathrm{H}-\mathrm{H}}=8.0 \mathrm{~Hz}, 2 \mathrm{H}, \mathrm{H}-11\right), 7.30\left(\mathrm{~d},{ }^{3} \mathrm{~J}_{\mathrm{H}-\mathrm{H}}=8.0\right.$ $\mathrm{Hz}, 2 \mathrm{H}, \mathrm{H}-10), 6.13\left(\mathrm{q},{ }^{3} \mathrm{~J}_{\mathrm{H}-\mathrm{H}}=7.1 \mathrm{~Hz}, 1 \mathrm{H}, \mathrm{H}-2\right), 6.04\left(\mathrm{~d},{ }^{2} \mathrm{~J}_{\mathrm{H}-\mathrm{H}}=1.4 \mathrm{~Hz}, 1 \mathrm{H}, \mathrm{H}-5 \mathrm{~b}\right), 5.49\left(\mathrm{~d},{ }^{2} \mathrm{~J}_{\mathrm{H}-}\right.$ $\mathrm{H}=1.4 \mathrm{~Hz}, 1 \mathrm{H}, \mathrm{H}-5 \mathrm{a}), 4.11\left(\mathrm{q},{ }^{3} \mathrm{~J}_{\mathrm{H}-\mathrm{H}}=7.1 \mathrm{~Hz}, 2 \mathrm{H}, \mathrm{H}-7\right), 1.70\left(\mathrm{~d},{ }^{3} \mathrm{~J}_{\mathrm{H}-\mathrm{H}}=7.1 \mathrm{~Hz}, 3 \mathrm{H}, \mathrm{H}-1\right), 1.14$ $\left(\mathrm{t},{ }^{3} \mathrm{~J}_{\mathrm{H}-\mathrm{H}}=7.1 \mathrm{~Hz}, 3 \mathrm{H}, \mathrm{H}-8\right)$

${ }^{13} \mathrm{C}\left\{{ }^{1} \mathrm{H}\right\}$ NMR $\left(\mathrm{CDCl}_{3}, 101 \mathrm{MHz}\right) \delta(\mathrm{ppm})=167.1(\mathrm{C}-6), 143.5(\mathrm{C}-4), 142.4(\mathrm{C}-9), 138.1(\mathrm{C}-3)$, 129.7 (C-10), 129.2 (q, ${ }^{2} \mathrm{~J}_{\mathrm{F}-\mathrm{H}}=32.3 \mathrm{~Hz}, \mathrm{C}-12$ ), 128.7 (C-2), 125.2 (C-11), 125.2 (C-5), 125.2 $\left(\mathrm{q},{ }^{3} \mathrm{~J}_{\mathrm{F}-\mathrm{H}}=3.9 \mathrm{~Hz}, \mathrm{C}-11\right), 61.0$ (C-7), 15.4 (C-1), 14.1 (C-8)

${ }^{19} \mathrm{~F}\left\{{ }^{1} \mathrm{H}\right\}$ NMR $\left(\mathrm{CDCl}_{3}, 282 \mathrm{MHz}\right) \delta(\mathrm{ppm})=-62.5$

LRMS $\left(\mathrm{ESI}^{+}\right)$calculated for $\mathrm{C}_{15} \mathrm{H}_{15} \mathrm{~F}_{3} \mathrm{O}_{2}[\mathrm{M}+\mathrm{H}]^{+}=285.1$; found $=285.1$

Ethyl (Z)-2-methylene-3-(4-(trifluoromethyl)phenyl)pent-3-enoate (Z)-6d

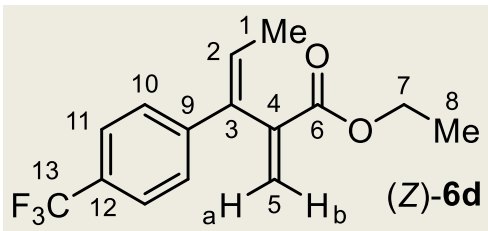

Obtained as minor product from the synthesis of $(E)-6 \mathbf{b}(1.6: 1$ $(E) /(Z)(2.6: 1$ in the crude $))$.

${ }^{1} \mathrm{H}$ NMR $\left(\mathrm{CDCl}_{3}, 400 \mathrm{MHz}\right) \delta(\mathrm{ppm})=7.53\left(\mathrm{~d},{ }^{3} \mathrm{~J}_{\mathrm{H}-\mathrm{H}}=8.2 \mathrm{~Hz}\right.$, $2 \mathrm{H}, \mathrm{H}-11), 7.40\left(\mathrm{~d},{ }^{3} \mathrm{~J}_{\mathrm{H}-\mathrm{H}}=8.1 \mathrm{~Hz}, 2 \mathrm{H}, \mathrm{H}-10\right), 6.65\left(\mathrm{~d},{ }^{2} \mathrm{~J}_{\mathrm{H}-\mathrm{H}}=\right.$ $1.8 \mathrm{~Hz}, 1 \mathrm{H}, \mathrm{H}-5 \mathrm{~b}$ ), $6.21\left(\mathrm{q},{ }^{3} \mathrm{~J}_{\mathrm{H}-\mathrm{H}}=7.0 \mathrm{~Hz}, 1 \mathrm{H}, \mathrm{H}-2\right), 5.72\left(\mathrm{~d},{ }^{2} \mathrm{~J}_{\mathrm{H}-\mathrm{H}}=1.8 \mathrm{~Hz}, 1 \mathrm{H}, \mathrm{H}-5 \mathrm{a}\right), 4.11$ (q, $\left.{ }^{3} \mathrm{~J}_{\mathrm{H}-\mathrm{H}}=7.1 \mathrm{~Hz}, 2 \mathrm{H}, \mathrm{H}-7\right), 1.84\left(\mathrm{~d},{ }^{3} \mathrm{~J}_{\mathrm{H}-\mathrm{H}}=7.0 \mathrm{~Hz}, 3 \mathrm{H}, \mathrm{H}-1\right), 1.13\left(\mathrm{t},{ }^{3} \mathrm{~J}_{\mathrm{H}-\mathrm{H}}=7.1 \mathrm{~Hz}, 3 \mathrm{H}, \mathrm{H}-8\right)$

${ }^{13} \mathrm{C}\left\{{ }^{1} \mathrm{H}\right\} \operatorname{NMR}\left(\mathrm{CDCl}_{3}, 101 \mathrm{MHz}\right) \delta(\mathrm{ppm})=166.3(\mathrm{C}-6), 144.7(\mathrm{C}-9), 138.4(\mathrm{C}-4), 137.6(\mathrm{C}-3)$, 130.3 (C-5), 128.6 (C-2), 126.4 (C-10), 125.4 (q, $\left.{ }^{3} \mathrm{~J}_{\mathrm{F}-\mathrm{H}}=3.4 \mathrm{~Hz}, \mathrm{C}-11\right), 61.0$ (C-7), 15.9 (C-1), $14.1(\mathrm{C}-8)$ 


\section{Ethyl $(E)$-2-methylene-3-(naphthalen-2-yl)pent-3-enoate $(E)-6 \mathbf{e}$}

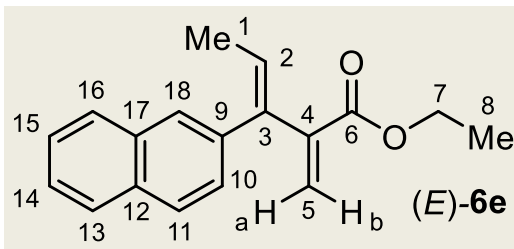

Synthesized according to general procedure B starting from a racemic mixture of $3 e$ and $4 \mathbf{e}$ isomers. Obtained as a colorless oil after flash chromatography using pentane/Et $\mathrm{t}_{2} \mathrm{O}$ 98.5:1.5 as eluent (33.3 $\mathrm{mg}, 50 \%$ yield, 8.6:1 $(E) /(Z)(4.7: 1$ in the crude), $91 \%$ purity). The VCP mixture was obtained according to the general cyclopropanation procedure $\left(9.3: 1 \mathrm{rr}_{(3 \mathrm{e} / 4 \mathrm{e})}, 1.4: 1 \mathrm{dr}\right.$ (cis/trans) $)$.

${ }^{1} \mathrm{H}$ NMR $\left(\mathrm{CDCl}_{3}, 400 \mathrm{MHz}\right) \delta(\mathrm{ppm})=7.87-7.75(\mathrm{~m}, 3 \mathrm{H}, \mathrm{H}-11+\mathrm{H}-13+\mathrm{H}-16), 7.65-7.62(\mathrm{~m}, 1 \mathrm{H}$, $\mathrm{H}-18)$, 7.50-7.43 (m, 2H, H-14+H-15), $7.34\left(\mathrm{dd},{ }^{3} \mathrm{~J}_{\mathrm{H}-\mathrm{H}}=8.4 \mathrm{~Hz},{ }^{4} \mathrm{~J}_{\mathrm{H}-\mathrm{H}}=1.7 \mathrm{~Hz}, 1 \mathrm{H}, \mathrm{H}-10\right), 6.17$ $\left(\mathrm{q},{ }^{3} \mathrm{~J}_{\mathrm{H}-\mathrm{H}}=7.1 \mathrm{~Hz} 1 \mathrm{H}, \mathrm{H}-2\right), 6.01\left(\mathrm{~d},{ }^{2} \mathrm{~J}_{\mathrm{H}-\mathrm{H}}=1.6 \mathrm{~Hz}, 1 \mathrm{H}, \mathrm{H}-5 \mathrm{~b}\right), 5.49\left(\mathrm{~d},{ }^{2} \mathrm{~J}_{\mathrm{H}-\mathrm{H}}=1.6 \mathrm{~Hz}, 1 \mathrm{H}, \mathrm{H}-\right.$ $5 a), 4.11\left(\mathrm{q},{ }^{3} \mathrm{~J}_{\mathrm{H}-\mathrm{H}}=7.1 \mathrm{~Hz}, 2 \mathrm{H}, \mathrm{H}-7\right), 1.75\left(\mathrm{~d},{ }^{3} \mathrm{~J}_{\mathrm{H}-\mathrm{H}}=7.1 \mathrm{~Hz}, 3 \mathrm{H}, \mathrm{H}-1\right), 1.13\left(\mathrm{t},{ }^{3} \mathrm{~J}_{\mathrm{H}-\mathrm{H}}=7.1 \mathrm{~Hz}\right.$, $3 \mathrm{H}, \mathrm{H}-8)$

${ }^{13} \mathrm{C}\left\{{ }^{1} \mathrm{H}\right\}$ NMR $\left(\mathrm{CDCl}_{3}, 101 \mathrm{MHz}\right) \delta(\mathrm{ppm})=167.6(\mathrm{C}-6), 144.1$ (C-4), 139.1 (C-3), $136.0(\mathrm{C}-9)$, 133.4 (C-17), 132.6 (C-12), 128.2-127.7 (C-2+C-10+C-11+C-13+C-16+C-18), 126.1 (C-14/C15), 125.9 (C-14/C-15), 124.4 (C-5), 60.9 (C-7), 15.5 (C-1), 14.1 (C-8)

HRMS $\left(E S{ }^{+}\right)$calculated for $\mathrm{C}_{18} \mathrm{H}_{19} \mathrm{O}_{2}[\mathrm{M}+\mathrm{H}]^{+}=267.1380$; found $=267.1391$

IR (neat) $\vee\left(\mathrm{cm}^{-1}\right)=2979,1717,1190,1021,907$

\section{Ethyl (Z)-2-methylene-3-(naphthalen-2-yl)pent-3-enoate (Z)-6e}

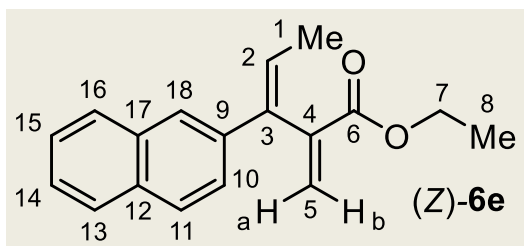

8.6 Hz, $\left.{ }^{4} \mathrm{~J}_{\mathrm{H}-\mathrm{H}}=1.9 \mathrm{~Hz}, 1 \mathrm{H}, \mathrm{H}-10\right), 7.50-7.43(\mathrm{~m}, 2 \mathrm{H}, \mathrm{H}-14+\mathrm{H}-15), 6.68\left(\mathrm{~d},{ }^{2} \mathrm{~J}_{\mathrm{H}-\mathrm{H}}=1.9 \mathrm{~Hz}, 1 \mathrm{H}\right.$, $\mathrm{H}-5 \mathrm{~b}), 6.29\left(\mathrm{q},{ }^{3} \mathrm{~J}_{\mathrm{H}-\mathrm{H}}=7.0 \mathrm{~Hz}, 1 \mathrm{H}, \mathrm{H}-2\right), 5.75\left(\mathrm{~d},{ }^{2} \mathrm{~J}_{\mathrm{H}-\mathrm{H}}=1.9 \mathrm{~Hz}, 1 \mathrm{H}, \mathrm{H}-5 \mathrm{a}\right), 4.11\left(\mathrm{q},{ }^{3} \mathrm{~J}_{\mathrm{H}-\mathrm{H}}=7.1\right.$ $\mathrm{Hz}, 2 \mathrm{H}, \mathrm{H}-7), 1.87\left(\mathrm{~d},{ }^{3} \mathrm{~J}_{\mathrm{H}-\mathrm{H}}=7.0 \mathrm{~Hz}, 3 \mathrm{H}, \mathrm{H}-1\right), 1.11\left(\mathrm{t},{ }^{3} \mathrm{~J}_{\mathrm{H}-\mathrm{H}}=7.1 \mathrm{~Hz}, 3 \mathrm{H}, \mathrm{H}-8\right)$

${ }^{13} \mathrm{C}\left\{{ }^{1} \mathrm{H}\right\}$ NMR $\left(\mathrm{CDCl}_{3}, 101 \mathrm{MHz}\right) \delta(\mathrm{ppm})=166.7(\mathrm{C}-6), 138.9(\mathrm{C}-4), 138.5(\mathrm{C}-9), 138.4(\mathrm{C}-3)$, 133.6 (C-17), 132.7 (C-12), 129.8 (C-5), 128.2-127.7 (C-11+C-13+C-16), 126.8 (C-2), 126.1 (C14/C-15), 125.7 (C-14/C-15), 124.6 (C-18), 124.4 (C-10), 60.9 (C-7), 15.9 (C-1), 14.1 (C-8) 
Tert-butyl (E)-3-(2-(ethoxycarbonyl)penta-1,3-dien-3-yl)-indole-1-carboxylate $(E)-6 \mathbf{f}$

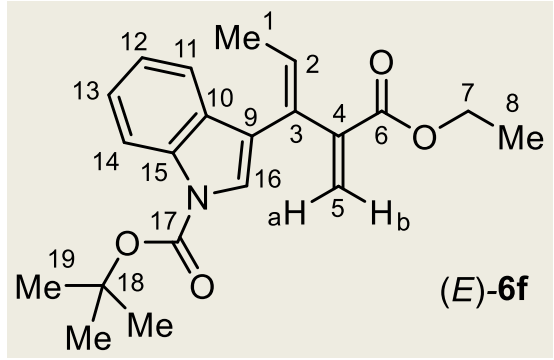

Synthesized according to general procedure B starting from a racemic mixture of $\mathbf{3 f}$ and $\mathbf{4 f}$ isomers (73\% conversion). Obtained as a colorless oil after flash chromatography using pentane/Et ${ }_{2} \mathrm{O} 97.5: 2.5$ as eluent $(4: 1(E) /(Z)(3.6: 1$ in the crude), contains $17 \%$ of trans-3f). The VCP mixture was obtained according to the general cyclopropanation procedure $\left.\left(8: 1 \mathrm{rr}_{(3 \mathrm{f} / 4 \mathrm{f})}, 1.9: 1 \mathrm{dr} \mathrm{(cis/trans}\right)\right) .{ }^{1} \mathrm{H}$ NMR $\left(\mathrm{CDCl}_{3}, 400 \mathrm{MHz}\right) \delta(\mathrm{ppm})=8.22-8.06(\mathrm{~m}, 1 \mathrm{H}$, $\mathrm{H}-14$ ), 7.44 (bs, $1 \mathrm{H}, \mathrm{H}-16$ ), 7.40 (ddd, ${ }^{3} \mathrm{~J}_{\mathrm{H}-\mathrm{H}}=7.8 \mathrm{~Hz},{ }^{4} \mathrm{~J}_{\mathrm{H}-\mathrm{H}}=1.1 \mathrm{~Hz},{ }^{4} \mathrm{~J}_{\mathrm{H}-\mathrm{H}}=0.8 \mathrm{~Hz}, 1 \mathrm{H}, \mathrm{H}-11$ ), 7.34-7.28 (m, 1H, H-13), 7.25-7.18 (m, 1H, H-12), 6.26 (q, $\left.{ }^{3} \mathrm{~J}_{\mathrm{H}-\mathrm{H}}=7.0 \mathrm{~Hz}, 1 \mathrm{H}, \mathrm{H}-2\right), 5.93$ (d, $\left.{ }^{2} \mathrm{~J}_{\mathrm{H}-\mathrm{H}}=1.4 \mathrm{~Hz}, 1 \mathrm{H}, \mathrm{H}-5 \mathrm{~b}\right), 5.51\left(\mathrm{~d},{ }^{2} \mathrm{~J}_{\mathrm{H}-\mathrm{H}}=1.4 \mathrm{~Hz}, 1 \mathrm{H}, \mathrm{H}-5 \mathrm{a}\right), 4.13\left(\mathrm{q},{ }^{3} \mathrm{~J}_{\mathrm{H}-\mathrm{H}}=7.1 \mathrm{~Hz}, 2 \mathrm{H}, \mathrm{H}-7\right)$, $1.72\left(\mathrm{~d},{ }^{3} \mathrm{~J}_{\mathrm{H}-\mathrm{H}}=7.0 \mathrm{~Hz}, 3 \mathrm{H}, \mathrm{H}-1\right), 1.68(\mathrm{~s}, 9 \mathrm{H}, \mathrm{H}-19), 1.13\left(\mathrm{t},{ }^{3} \mathrm{~J}_{\mathrm{H}-\mathrm{H}}=7.1 \mathrm{~Hz}, 3 \mathrm{H}, \mathrm{H}-8\right)$

${ }^{13} \mathrm{C}\left\{{ }^{1} \mathrm{H}\right\}$ NMR $\left(\mathrm{CDCl}_{3}, 101 \mathrm{MHz}\right) \delta(\mathrm{ppm})=167.7(\mathrm{C}-6), 149.9(\mathrm{C}-17), 143.4(\mathrm{C}-4), 135.4(\mathrm{C}-$ 15), 130.4 (C-2), 130.3 (C-3), 130.2 (C-10), 1124.7 (C-16), 124.4 (C-13), 123.6 (C-5), 122.7 (C-12), 120.7 (C-11), 118.2 (C-9), 115.3 (C-14), 83.9 (C-18), 60.9 (C-7), 28.4 (C-19), 16.0 (C1), $14.1(\mathrm{C}-8)$

LRMS $\left(\mathrm{ESI}^{+}\right)$calculated for $\mathrm{C}_{21} \mathrm{H}_{25} \mathrm{NO}_{4}[\mathrm{M}+\mathrm{H}]^{+}=356.2 ;$ found $=356.2$

tert-butyl (Z)-3-(2-(ethoxycarbonyl)penta-1,3-dien-3-yl)-indole-1-carboxylate $(Z)-6 f$

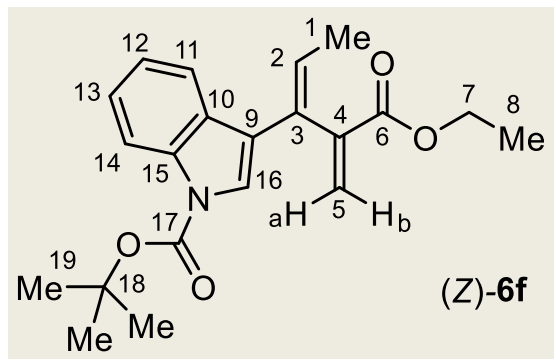
$\mathrm{H}=1.8 \mathrm{~Hz}, 1 \mathrm{H}, \mathrm{H}-5 \mathrm{a}), 4.15\left(\mathrm{q},{ }^{3} \mathrm{~J}_{\mathrm{H}-\mathrm{H}}=7.1 \mathrm{~Hz}, 2 \mathrm{H}, \mathrm{H}-7\right), 1.84\left(\mathrm{~d},{ }^{3} \mathrm{~J}_{\mathrm{H}-\mathrm{H}}=7.0 \mathrm{~Hz}, 3 \mathrm{H}, \mathrm{H}-1\right), 1.66$ (s, 9H, H-19), $1.16\left(\mathrm{t}, 3^{3} \mathrm{H}_{\mathrm{H}}=7.1 \mathrm{~Hz}, 3 \mathrm{H}, \mathrm{H}-8\right)$

${ }^{13} \mathrm{C}\left\{{ }^{1} \mathrm{H}\right\}$ NMR $\left(\mathrm{CDCl}_{3}, 101 \mathrm{MHz}\right) \delta(\mathrm{ppm})=166.7(\mathrm{C}-5), 149.8(\mathrm{C}-17), 139.3(\mathrm{C}-4), 131.0(\mathrm{C}-$ 3), 129.1 (C-10), 128.9 (C-5), 126.8 (C-2), 124.5 (C-13), 123.3 (C-16), 122.8 (C-12), 122.4 (C9), 120.4 (C-11), 115.4 (C-14), 83.8 (C-18), 61.0 (C-7), 28.4 (C-19), 15.4 (C-1), 14.2 (C-8)

Obtained as minor product from the synthesis of $(E)-6 f(4: 1$ $(E) /(Z)(3: 6: 1$ in the crude) $)$.

${ }^{1} \mathrm{H} \mathbf{N M R}\left(\mathrm{CDCl}_{3}, 400 \mathrm{MHz}\right) \delta(\mathrm{ppm})=8.22-8.06(\mathrm{~m}, 1 \mathrm{H}, \mathrm{H}-$ 14), 7.76-7.71 (m, 1H, H-11), 7.38 (bs, $1 \mathrm{H}, \mathrm{H}-16), 7.34-7.28$ (m, 1H, H-13), 7.25-7.18 (m, 1H, H-12), $6.54\left(\mathrm{~d},{ }^{2} \mathrm{~J}_{\mathrm{H}-\mathrm{H}}=1.8\right.$ $\mathrm{Hz}, 1 \mathrm{H}, \mathrm{H}-5 \mathrm{~b}), 6.25\left(\mathrm{q},{ }^{3} \mathrm{~J}_{\mathrm{H}-\mathrm{H}}=7.0 \mathrm{~Hz}, 1 \mathrm{H}, \mathrm{H}-2\right), 5.74\left(\mathrm{~d},{ }^{2} \mathrm{~J}_{\mathrm{H}-}\right.$ 
Ethyl $(E)$-2-methylene-3-(thiophen-3-yl)pent-3-enoate $(E)-6 \mathbf{g}$

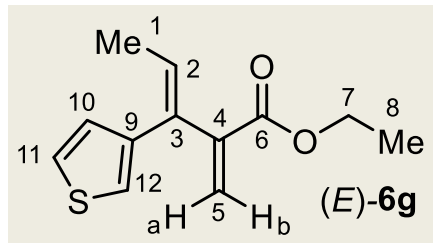

Synthesized according to general procedure B starting from a racemic mixture of $\mathbf{3} \mathbf{g}$ and $\mathbf{4} \mathbf{g}$ isomers. Obtained as a yellowish oil after flash chromatography using pentane/ $\mathrm{Et}_{2} \mathrm{O}$ 97:3 as eluent (20.6 mg, 37\% yield, 3.6:1 (E)/(Z) (4.7:1 in the crude), 96\% purity). The VCP mixture was obtained according to the general cyclopropanation procedure (9.3:1 $\mathrm{rr}$ $(3 \mathrm{~g} / 4 \mathrm{~g}), 1: 1.1 d r_{(\text {cis/trans) }) \text {. }}$

${ }^{1} \mathrm{H}$ NMR $\left(\mathrm{CDCl}_{3}, 400 \mathrm{MHz}\right) \delta(\mathrm{ppm})=7.29\left(\mathrm{dd},{ }^{3} \mathrm{~J}_{\mathrm{H}-\mathrm{H}}=4.9 \mathrm{~Hz},{ }^{4} \mathrm{~J}_{\mathrm{H}-\mathrm{H}}=3.0 \mathrm{~Hz}, 1 \mathrm{H}, \mathrm{H}-11\right), 7.07$ $\left(\mathrm{dd},{ }^{4} \mathrm{~J}_{\mathrm{H}-\mathrm{H}}=3.0 \mathrm{~Hz},{ }^{4} \mathrm{~J}_{\mathrm{H}-\mathrm{H}}=1.3 \mathrm{~Hz}, 1 \mathrm{H}, \mathrm{H}-12\right), 6.99\left(\mathrm{dd},{ }^{3} \mathrm{~J}_{\mathrm{H}-\mathrm{H}}=4.9 \mathrm{~Hz},{ }^{4} \mathrm{~J}_{\mathrm{H}-\mathrm{H}}=1.3 \mathrm{~Hz}, 1 \mathrm{H}, \mathrm{H}-\right.$ 10), $6.01\left(\mathrm{q},{ }^{3} \mathrm{~J}_{\mathrm{H}-\mathrm{H}}=7.1 \mathrm{~Hz}, 1 \mathrm{H}, \mathrm{H}-2\right), 5.98\left(\mathrm{~d},{ }^{3} \mathrm{~J}_{\mathrm{H}-\mathrm{H}}=1.7 \mathrm{~Hz}, 1 \mathrm{H}, \mathrm{H}-5 \mathrm{~b}\right), 5.51\left(\mathrm{~d},{ }^{3} \mathrm{~J}_{\mathrm{H}-\mathrm{H}}=1.7\right.$ $\mathrm{Hz}, 1 \mathrm{H}, \mathrm{H}-5 \mathrm{a}), 4.13\left(\mathrm{q},{ }^{3} \mathrm{~J}_{\mathrm{H}-\mathrm{H}}=7.1,2 \mathrm{H}, \mathrm{H}-7\right), 1.79\left(\mathrm{~d},{ }^{3} \mathrm{~J}_{\mathrm{H}-\mathrm{H}}=7.1,3 \mathrm{H}, \mathrm{H}-1\right), 1.115\left(\mathrm{t},{ }^{3} \mathrm{~J}_{\mathrm{H}-\mathrm{H}}=7.1\right.$, $3 \mathrm{H}, \mathrm{H}-8)$

${ }^{13} \mathrm{C}\left\{{ }^{1} \mathrm{H}\right\}$ NMR $\left(\mathrm{CDCl}_{3}, 101 \mathrm{MHz}\right) \delta(\mathrm{ppm})=167.6(\mathrm{C}-6), 144.1(\mathrm{C}-4), 138.8(\mathrm{C}-9), 134.1(\mathrm{C}-3)$, 128.8 (C-10), 127.9 (C-2), 124.9 (C-11), 124.2 (C-5), 123.4 (C-12), 60.9 (C-7), 15.6 (C-1), 14.1 $(\mathrm{C}-8)$

HRMS $\left(\mathrm{ESI}^{+}\right)$calculated for $\mathrm{C}_{12} \mathrm{H}_{15} \mathrm{O}_{2} \mathrm{~S}[\mathrm{M}+\mathrm{H}]^{+}=223.0788$; found $=223.0797$

IR (neat) $\vee\left(\mathrm{cm}^{-1}\right)=2925,1718,1188,1134,1024$

\section{Ethyl $(E)$-2-methylene-3-(thiophen-3-yl)pent-3-enoate $(Z)-6 \mathrm{~g}$}

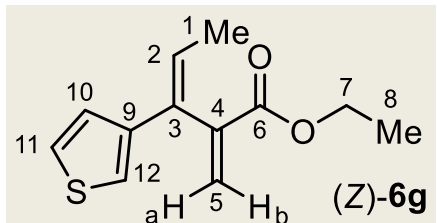

$(Z)-6 g$

Obtained as minor product from the synthesis of $(E)-6 \mathbf{g}(37 \%$ yield, $3.6: 1(E) /(Z)(4.7: 1$ in the crude)).

${ }^{1} \mathrm{H}$ NMR $\left(\mathrm{CDCl}_{3}, 400 \mathrm{MHz}\right) \delta(\mathrm{ppm})=7.24\left(\mathrm{dd},{ }^{3} \mathrm{~J}_{\mathrm{H}-\mathrm{H}}=5.1 \mathrm{~Hz},{ }^{4} \mathrm{~J}_{\mathrm{H}-}\right.$ $\mathrm{H}=3.0 \mathrm{~Hz}, 1 \mathrm{H}, \mathrm{H}-11), 7.17\left(\mathrm{dd},{ }^{3} \mathrm{~J}_{\mathrm{H}-\mathrm{H}}=5.1 \mathrm{~Hz},{ }^{4} \mathrm{~J}_{\mathrm{H}-\mathrm{H}}=1.3 \mathrm{~Hz}, 1 \mathrm{H}\right.$, $\mathrm{H}-10), 6.94\left(\mathrm{dd},{ }^{4} \mathrm{~J}_{\mathrm{H}-\mathrm{H}}=2.9 \mathrm{~Hz},{ }^{4} \mathrm{~J}_{\mathrm{H}-\mathrm{H}}=1.3 \mathrm{~Hz}, 1 \mathrm{H}, \mathrm{H}-12\right), 6.56\left(\mathrm{~d},{ }^{3} \mathrm{~J}_{\mathrm{H}-\mathrm{H}}=1.9 \mathrm{~Hz}, 1 \mathrm{H}, \mathrm{H}-5 \mathrm{~b}\right)$, $6.16\left(\mathrm{q},{ }^{3} \mathrm{~J}_{\mathrm{H}-\mathrm{H}}=7.0 \mathrm{~Hz}, 1 \mathrm{H}, \mathrm{H}-2\right), 5.67\left(\mathrm{~d},{ }^{3} \mathrm{~J}_{\mathrm{H}-\mathrm{H}}=1.9 \mathrm{~Hz}, 1 \mathrm{H}, \mathrm{H}-5 \mathrm{a}\right), 4.17\left(\mathrm{q},{ }^{3} \mathrm{~J}_{\mathrm{H}-\mathrm{H}}=7.1 \mathrm{~Hz}, 2 \mathrm{H}\right.$, $\mathrm{H}-7), 1.76\left(\mathrm{~d},{ }^{3} \mathrm{~J}_{\mathrm{H}-\mathrm{H}}=7.0 \mathrm{~Hz}, 3 \mathrm{H}, \mathrm{H}-1\right), 1.19\left(\mathrm{t},{ }^{3} \mathrm{~J}_{\mathrm{H}-\mathrm{H}}=7.1 \mathrm{~Hz}, 3 \mathrm{H}, \mathrm{H}-8\right)$

${ }^{13} \mathrm{C}\left\{{ }^{1} \mathrm{H}\right\}$ NMR $\left(\mathrm{CDCl}_{3}, 101 \mathrm{MHz}\right) \delta(\mathrm{ppm})=166.6(\mathrm{C}-6), 142.7(\mathrm{C}-9), 139.1$ (C-4), $133.5(\mathrm{C}-3)$, 129.2 (C-5), 125.7 (C-10), 125.4 (C-11), 124.8 (C-2), 120.2 (C-12), 61.0 (C-7), 15.2 (C-1), 14.2 $(\mathrm{C}-8)$ 
Ethyl (E)-2-methylene-3-phenethylpent-3-enoate $(E)-6 \mathbf{h}$

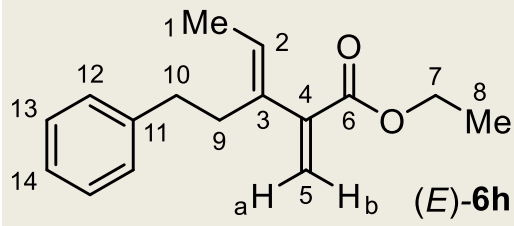

Synthesized according to general procedure B starting from a racemic mixture of $\mathbf{3 h}$ and $\mathbf{4 h}$ isomers. Obtained as a colorless oil after flash chromatography using pentane/Et $\mathrm{t}_{2} \mathrm{O}$ 98.5:1.5 as eluent $(26.9 \mathrm{mg}, 44 \%$ yield, $1: 1(E) /(Z), 91 \%$ purity). The VCP mixture was obtained according to the general cyclopropanation procedure $\left(4.4: 1 r_{(3 h / 4 h)}, 1.4: 1 d r_{(c i s / t r a n s)}\right)$.

${ }^{1} \mathrm{H}$ NMR $\left(\mathrm{CDCl}_{3}, 400 \mathrm{MHz}\right) \delta(\mathrm{ppm})=7.31-7.23(\mathrm{~m}, 2 \mathrm{H}, \mathrm{H}-13), 7.22-7.14(\mathrm{~m}, 3 \mathrm{H}, \mathrm{H}-12+\mathrm{H}-14)$, $5.93\left(\mathrm{~d},{ }^{2} \mathrm{~J}_{\mathrm{H}-\mathrm{H}}=1.5 \mathrm{~Hz}, 1 \mathrm{H}, \mathrm{H}-5 \mathrm{~b}\right), 5.65\left(\mathrm{q},{ }^{3} \mathrm{~J}_{\mathrm{H}-\mathrm{H}}=6.9 \mathrm{~Hz}, 1 \mathrm{H}, \mathrm{H}-2\right), 5.54\left(\mathrm{~d},{ }^{2} \mathrm{~J}_{\mathrm{H}-\mathrm{H}}=1.5 \mathrm{~Hz}, \mathrm{H}-\right.$ 5a), $4.24\left(\mathrm{q},{ }^{3} \mathrm{~J}_{\mathrm{H}-\mathrm{H}}=7.1 \mathrm{~Hz}, 2 \mathrm{H}, \mathrm{H}-7\right), 2.70-2.55(\mathrm{~m}, 4 \mathrm{H}, \mathrm{H}-9+\mathrm{H}-10), 1.61\left(\mathrm{~d},{ }^{3} \mathrm{~J}_{\mathrm{H}-\mathrm{H}}=6.9 \mathrm{~Hz}\right.$, $3 \mathrm{H}, \mathrm{H}-1), 1.32\left(\mathrm{t},{ }^{3} \mathrm{~J}_{\mathrm{H}-\mathrm{H}}=7.1 \mathrm{~Hz}, 3 \mathrm{H}, \mathrm{H}-8\right)$

${ }^{13} \mathrm{C}\left\{{ }^{1} \mathrm{H}\right\}$ NMR $\left(\mathrm{CDCl}_{3}, 101 \mathrm{MHz}\right) \delta(\mathrm{ppm})=167.8$ (C-6), 144.1 (C-4), 142.2/142.1 (C-11), 137.4/137.3 (C-3), 128.6/128.5 (C-12), 128.4 (C-13), 126.5 (C-2), 126.0/125.9 (C-14), 123.0 (C-5), 61.0 (C-7), 34.5 (C-10), 30.6 (C-9), 14.4 (C-8), 13.8 (C-1)

HRMS $\left(\mathrm{ESI}^{+}\right)$calculated for $\mathrm{C}_{16} \mathrm{H}_{21} \mathrm{O}_{2}[\mathrm{M}+\mathrm{H}]^{+}=245.1537$; found $=245.1543$

\section{Ethyl (Z)-2-methylene-3-phenethylpent-3-enoate (Z)-6h}<smiles>C=C(C(=O)OCC(C)O)/C(=C\C)CCc1ccccc1</smiles>

Obtained in a 1:1 ratio along with $(E)-6 \mathrm{~h}$ (44\% yield, $1: 1$ $(E) /(Z), 91 \%$ purity).

${ }^{1} \mathrm{H}$ NMR $\left(\mathrm{CDCl}_{3}, 400 \mathrm{MHz}\right) \delta(\mathrm{ppm})=7.31-7.23(\mathrm{~m}, 2 \mathrm{H}, \mathrm{H}-$ 13), 7.22-7.14 (m, 3H, H-12+H-14), $6.39\left(\mathrm{~d},{ }^{2} \mathrm{~J}_{\mathrm{H}-\mathrm{H}}=2.0 \mathrm{~Hz}\right.$, $1 \mathrm{H}, \mathrm{H}-5 \mathrm{~b}), 5.56-5.50(\mathrm{~m}, 1 \mathrm{H}, \mathrm{H}-2), 5.50\left(\mathrm{~d},{ }^{2} \mathrm{~J}_{\mathrm{H}-\mathrm{H}}=1.9 \mathrm{~Hz}, 1 \mathrm{H}, \mathrm{H}-5 \mathrm{a}\right), 4.23\left(\mathrm{q},{ }^{3} \mathrm{~J}_{\mathrm{H}-\mathrm{H}}=7.1 \mathrm{~Hz}\right.$, $2 \mathrm{H}, \mathrm{H}-7), 2.70-2.55(\mathrm{~m}, 2 \mathrm{H}, \mathrm{H}-10), 2.53-2.46(\mathrm{~m}, 2 \mathrm{H}, \mathrm{H}-9), 1.57\left(\mathrm{~d},{ }^{3} \mathrm{~J}_{\mathrm{H}-\mathrm{H}}=6.8 \mathrm{~Hz}, 3 \mathrm{H}, \mathrm{H}-1\right)$, $1.31\left(\mathrm{t},{ }^{3} \mathrm{~J}_{\mathrm{H}-\mathrm{H}}=7.1 \mathrm{~Hz}, 3 \mathrm{H}, \mathrm{H}-8\right)$

${ }^{13} \mathrm{C}\left\{{ }^{1} \mathrm{H}\right\}$ NMR $\left(\mathrm{CDCl}_{3}, 101 \mathrm{MHz}\right) \delta(\mathrm{ppm})=166.7$ (C-6), 142.2/142.1 (C-11), 139.9 (C-4), 137.4/137.3 (C-3), 128.6/128.5 (C-12), 128.4 (C-13), 127.9 (C-5), 126.0/125.9 (C-14), 124.1 (C-2), 61.0 (C-7), 39.2 (C-9), 34.8 (C-10), 14.8 (C-1), 14.4 (C-8)

HRMS $\left(\mathrm{ESI}^{+}\right)$calculated for $\mathrm{C}_{16} \mathrm{H}_{21} \mathrm{O}_{2}[\mathrm{M}+\mathrm{H}]^{+}=245.1537$; found $=245.1543$ 
(E)-4-([1,1'-biphenyl]-4-yl)-2-methyl-3-methylenehex-4-en-2-ol $(E)-9$<smiles>C/C=C(\c1ccc(-c2ccccc2)cc1)C(C)(C)O</smiles>

$(E)-9$

Synthesized according to general procedure B at $110^{\circ} \mathrm{C}$ for 72 hours, starting from 7. Obtained as an off-white solid after flash chromatography using pentane/ $\mathrm{Et}_{2} \mathrm{O} 90: 10$ as eluent $(41.8 \mathrm{mg}$, $81 \%$ yield, $4.1: 1(E, E) /(E, Z)(3.6: 1$ in the crude), $>99 \%$ purity). VCP 7 was obtained as described for the synthesis of compound 8 in Section 2.3 (2.4:1 dr (cis/trans) $)$, starting from racemic $3 a$.

${ }^{1} \mathrm{H}$ NMR $\left(\mathrm{CDCl}_{3}, 400 \mathrm{MHz}\right) \delta(\mathrm{ppm})=7.64-7.60(\mathrm{~m}, 2 \mathrm{H}, \mathrm{H}-14), 7.60-7.56(\mathrm{~m}, 2 \mathrm{H}, \mathrm{H}-11), 7.47-$ $7.40(\mathrm{~m}, 2 \mathrm{H}, \mathrm{H}-15), 7.37-7.30(\mathrm{~m}, 3 \mathrm{H}, \mathrm{H}-10+\mathrm{H}-16), 5.82\left(\mathrm{q},{ }^{3} \mathrm{~J}_{\mathrm{H}-\mathrm{H}}=7.1 \mathrm{~Hz}, 1 \mathrm{H}, \mathrm{H}-2\right), 5.32(\mathrm{~d}$, $\left.{ }^{2} \mathrm{~J}_{\mathrm{H}-\mathrm{H}}=2.0 \mathrm{~Hz}, 1 \mathrm{H}, \mathrm{H}-5 \mathrm{~b}\right), 5.08\left(\mathrm{~d},{ }^{2} \mathrm{~J}_{\mathrm{H}-\mathrm{H}}=2.0 \mathrm{~Hz}, 1 \mathrm{H}, \mathrm{H}-5 \mathrm{a}\right), 1.75\left(\mathrm{~d},{ }^{3} \mathrm{~J}_{\mathrm{H}-\mathrm{H}}=7.1 \mathrm{~Hz}, 3 \mathrm{H}, \mathrm{H}-1\right)$, $1,47(\mathrm{~s}, 1 \mathrm{H}, \mathrm{H}-7), 1.23(\mathrm{~s}, 6 \mathrm{H}, \mathrm{H}-8)$

${ }^{13} \mathrm{C}\left\{{ }^{1} \mathrm{H}\right\}$ NMR $\left(\mathrm{CDCl}_{3}, 101 \mathrm{MHz}\right) \delta(\mathrm{ppm})=159.5(\mathrm{C}-4), 142.3(\mathrm{C}-3), 140.9(\mathrm{C}-13), 139.8(\mathrm{C}-$ 12), 129.8 (C-10) 128.9 (C-15), 127.4 (C-16), 127.1 (C-14), 126.8 (C-11), 125.9 (C-2), 112.9 (C-5), 73.4 (C-6), 30.7 (C-8), 15.2 (C-1)

MP $\left({ }^{\circ} \mathrm{C}\right)=69-73$

HRMS $\left(\mathrm{ESI}^{+}\right)$calculated for $\mathrm{C}_{20} \mathrm{H}_{21}[\mathrm{M}-\mathrm{OH}]^{+}=261.1638 ;$ found $=261.1650$

IR (neat) $\vee\left(\mathrm{cm}^{-1}\right)=3399,2977,1486,1362,1134,914$

(Z)-4-([1,1'-biphenyl]-4-yl)-2-methyl-3-methylenehex-4-en-2-ol (Z)-9<smiles>CC=CC(=CC)C(=CC)c1ccc(-c2ccccc2)cc1</smiles>

$(Z)-9$

Obtained as minor product from the synthesis of $(E)-9(56.4$ $\mathrm{mg}, 81 \%$ yield, $4.1: 1(E) /(Z)(3.6: 1$ in the crude).

${ }^{1} \mathrm{H}$ NMR $\left(\mathrm{CDCl}_{3}, 400 \mathrm{MHz}\right) \delta(\mathrm{ppm})=7.64-7.56(\mathrm{~m}, 2 \mathrm{H}, \mathrm{H}-14)$, 7.55-7.51 (m, 2H, $\mathrm{H}-11), 7.47-7.40(\mathrm{~m}, 2 \mathrm{H}, \mathrm{H}-10), 7.37-7.30$ (m, $1 \mathrm{H}, \mathrm{H}-16), 6.01\left(\mathrm{q},{ }^{3} \mathrm{~J}_{\mathrm{H}-\mathrm{H}}=6.9 \mathrm{~Hz}, 1 \mathrm{H}, \mathrm{H}-2\right), 5.66\left(\mathrm{~d},{ }^{2} \mathrm{~J}_{\mathrm{H}-\mathrm{H}}=1.8\right.$ $\mathrm{Hz}, 1 \mathrm{H}, \mathrm{H}-5 \mathrm{~b}), 5.04\left(\mathrm{~d},{ }^{2} \mathrm{~J}_{\mathrm{H}-\mathrm{H}}=1.8 \mathrm{~Hz}, 1 \mathrm{H}, \mathrm{H}-5 \mathrm{a}\right), 1.85\left(\mathrm{~d},{ }^{3} \mathrm{~J}_{\mathrm{H}-\mathrm{H}}=6.8 \mathrm{~Hz}, 3 \mathrm{H}, \mathrm{H}-1\right), 1.50(\mathrm{~s}, 1 \mathrm{H}$, $\mathrm{H}-7), 1.26(\mathrm{~s}, 6 \mathrm{H}, \mathrm{H}-8)$

${ }^{13} \mathrm{C}\left\{{ }^{1} \mathrm{H}\right\}$ NMR $\left(\mathrm{CDCl}_{3}, 101 \mathrm{MHz}\right) \delta(\mathrm{ppm})=153.1(\mathrm{C}-4), 142.1(\mathrm{C}-3), 141.1(\mathrm{C}-9), 140.9$ (C13), 139.8 (C-12), 128.9 (C-15), 127.3 (C-16), 127.1 (C-14), 127.1 (C-11), 126.9 (C-10), 126.4 (C-2), 30.5 (C-8), 16.3 (C-1) 
Ethyl trans-4-(4-isobutylphenyl)-3-methyl-2-phenyl-3,6-dihydro-2H-1,2-oxazine-6-carboxylate 11

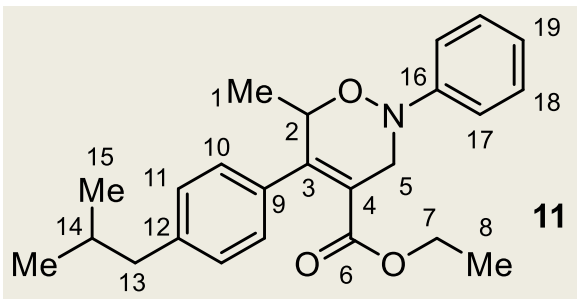

A sample of $6 \mathbf{c}(4.8: 1(E) /(Z), 68.1 \mathrm{mg}, 0.25 \mathrm{mmol})$ was dissolved in $2.5 \mathrm{ml}$ of tetrahydrofuran. Nitrosobenzene ( $53.6 \mathrm{mg}, 0.5 \mathrm{mmol}$ ) was added and the reaction mixture was stirred at $60{ }^{\circ} \mathrm{C}$ for 18 hours. Obtained as a yellow solid after flash chromatography using pentane/ $\mathrm{Et}_{2} \mathrm{O} 96: 4$ as eluent (11+12: $69.3 \mathrm{mg}, 73 \%$ yield, 3.7:1 rr).

${ }^{1} \mathrm{H}$ NMR $\left(\mathrm{CDCl}_{3}, 400 \mathrm{MHz}\right) \delta(\mathrm{ppm})=7.38-7.31(\mathrm{~m}, 2 \mathrm{H}, \mathrm{H}-18), 7.23-7.18(\mathrm{~m}, 2 \mathrm{H}, \mathrm{H}-17), 7.16-$ $7.11(\mathrm{~m}, 2 \mathrm{H}, \mathrm{H}-11)$, 7.08-7.05 (m, 2H, H-10), 7.05-7.00 (m, 1H, H-19), 4.90-4.83 (m, 1H, H-2), $4.36\left(\mathrm{dd},{ }^{2} \mathrm{~J}_{\mathrm{H}-\mathrm{H}}=16.0 \mathrm{~Hz},{ }^{5} \mathrm{~J}_{\mathrm{H}-\mathrm{H}}=1.5 \mathrm{~Hz}, 1 \mathrm{H}, \mathrm{H}-5 \mathrm{a}\right), 4.02-3.88(\mathrm{~m}, 3 \mathrm{H}, \mathrm{H}-5 \mathrm{~b}+\mathrm{H}-7), 2.49\left(\mathrm{~d},{ }^{3} \mathrm{~J}_{\mathrm{H}-}\right.$ $\mathrm{H}=7.2 \mathrm{~Hz}, 2 \mathrm{H}, \mathrm{H}-13), 1.94-1.81(\mathrm{~m}, 1 \mathrm{H}, \mathrm{H}-14), 1.33\left(\mathrm{~d},{ }^{3} \mathrm{~J}_{\mathrm{H}-\mathrm{H}}=6.6 \mathrm{~Hz}, 3 \mathrm{H}, \mathrm{H}-1\right), 0.96-0.88(\mathrm{~m}$, $9 \mathrm{H}, \mathrm{H}-8+\mathrm{H}-15)$

${ }^{13} \mathrm{C}\left\{{ }^{1} \mathrm{H}\right\}$ NMR $\left(\mathrm{CDCl}_{3}, 101 \mathrm{MHz}\right) \delta(\mathrm{ppm})=166.5(\mathrm{C}-6), 152.1(\mathrm{C}-3), 150.2(\mathrm{C}-16), 141.5(\mathrm{C}-$ 12), 135.6 (C-9), 129.0 (C-11/C-18), 129.0 (C-11/C-18), 127.4 (C-10), 123.4 (C-3), 122.5C19), 116.0 (C-17), 77.5 (C-2), 60.6 (C-7), 51.6 (C-5), 45.3 (C-13), 30.4 (C-14), 22.5 (C-15), 17.8 (C-1), $13.7(\mathrm{C}-8)$.

$\operatorname{MP}\left({ }^{\circ} \mathrm{C}\right)=76-80$

HRMS $\left(\mathrm{ESI}^{+}\right)$calculated for $\mathrm{C}_{24} \mathrm{H}_{29} \mathrm{NO}_{3}[\mathrm{M}+\mathrm{H}]^{+}=380.2226$; found $=380.2223$

IR (neat) $\vee\left(\mathrm{cm}^{-1}\right)=2956,1706,1598,1493,1373,1271,1154,1058$

Ethyl 4-(4-isobutylphenyl)-3-methyl-2-phenyl-3,6-dihydro-2H-1,2-oxazine-5-carboxylate 12

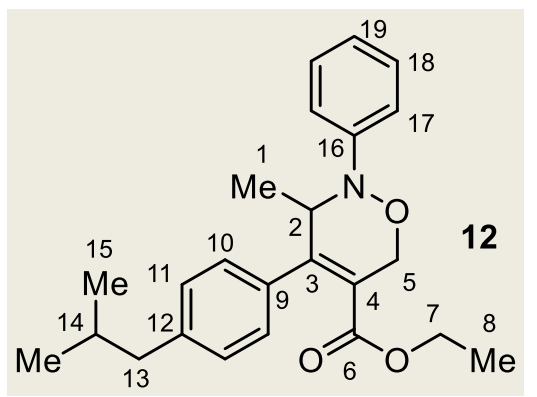
Obtained as minor product from the synthesis of $11(\mathbf{1 1 + 1 2}$ : $73 \%$ yield, 3.7:1 rr).

${ }^{1} \mathrm{H}$ NMR $\left(\mathrm{CDCl}_{3}, 400 \mathrm{MHz}\right) \delta(\mathrm{ppm})=7.34-7.29(\mathrm{~m}, 2 \mathrm{H}, \mathrm{H}-$ 18), 7.15-7.09 (m, 6H, H-10+H-11+H-17), 7.00-6.95 (m, $1 \mathrm{H}$, $\mathrm{H}-19), 4.82\left(\mathrm{~d},{ }^{2} \mathrm{~J}_{\mathrm{H}-\mathrm{H}}=15.8 \mathrm{~Hz}, 1 \mathrm{H}, \mathrm{H}-5 \mathrm{a}\right), 4.73\left(\mathrm{dd},{ }^{2} \mathrm{~J}_{\mathrm{H}-\mathrm{H}}=\right.$ $\left.15.6 \mathrm{~Hz},{ }^{5} \mathrm{~J}_{\mathrm{H}-\mathrm{H}}=2.1 \mathrm{~Hz}, 1 \mathrm{H}, \mathrm{H}-5 \mathrm{~b}\right), 4.40\left(\mathrm{dq},{ }^{3} \mathrm{~J}_{\mathrm{H}-\mathrm{H}}=6.7 \mathrm{~Hz}\right.$, $\left.{ }^{5} \mathrm{~J}_{\mathrm{H}-\mathrm{H}}=1.7 \mathrm{~Hz}, 1 \mathrm{H}, \mathrm{H}-2\right), 4.02-3.88(\mathrm{~m}, 2 \mathrm{H}, \mathrm{H} 7), 2.50\left(\mathrm{~d},{ }^{3} \mathrm{~J}_{\mathrm{H}-\mathrm{H}}=7.2 \mathrm{~Hz}, 2 \mathrm{H}, \mathrm{H}-13\right), 1.94-1.81$ $(\mathrm{m}, 1 \mathrm{H}, \mathrm{H}-14), 1.03\left(\mathrm{~d},{ }^{3} \mathrm{~J}_{\mathrm{H}-\mathrm{H}}=6.6 \mathrm{~Hz}, 3 \mathrm{H}, \mathrm{H}-1\right), 0.96-0.88(\mathrm{~m}, 9 \mathrm{H}, \mathrm{H}-8+\mathrm{H}-15)$

${ }^{13} \mathrm{C}\left\{{ }^{1} \mathrm{H}\right\}$ NMR $\left(\mathrm{CDCl}_{3}, 101 \mathrm{MHz}\right) \delta(\mathrm{ppm})=165.9(\mathrm{C}-6), 150.6$ (C-3), 148.1 (C-16), $141.7(\mathrm{C} 12)$, 136.6 (C-9), 129.1 (C-11/C-18) , 129.0 (C-11/C-18), 127.7 (C-10), 125.5 (C-4), 121.9 (C-19), 
115.9 (C-17), 68.6 (C-5), 60.4 (C-7), 59.7 (C-2), 45.3 (C-13), 30.4 (C-14), 22.5 (C-15), 13.7 (C-8), $12.5(\mathrm{C}-1)$

\section{References}

${ }^{1}$ Fiorito, D.; Folliet, S.; Liu, Y.; Mazet, C. ACS Catal. 2018, 8, 1392-1398.

${ }^{2}$ Li, C.; Shin, K.; Liu, R. Y.; Buchwald, S. L. Angew. Chem. Int. Ed. 2019, 58, 17074-17080.

${ }^{3}$ Garbo, M.; Besnard, C.; Guénée, L.; Mazet, C. ACS Catal. 2020, 10, 9604-9611. 
5. NMR spectra of new compounds 


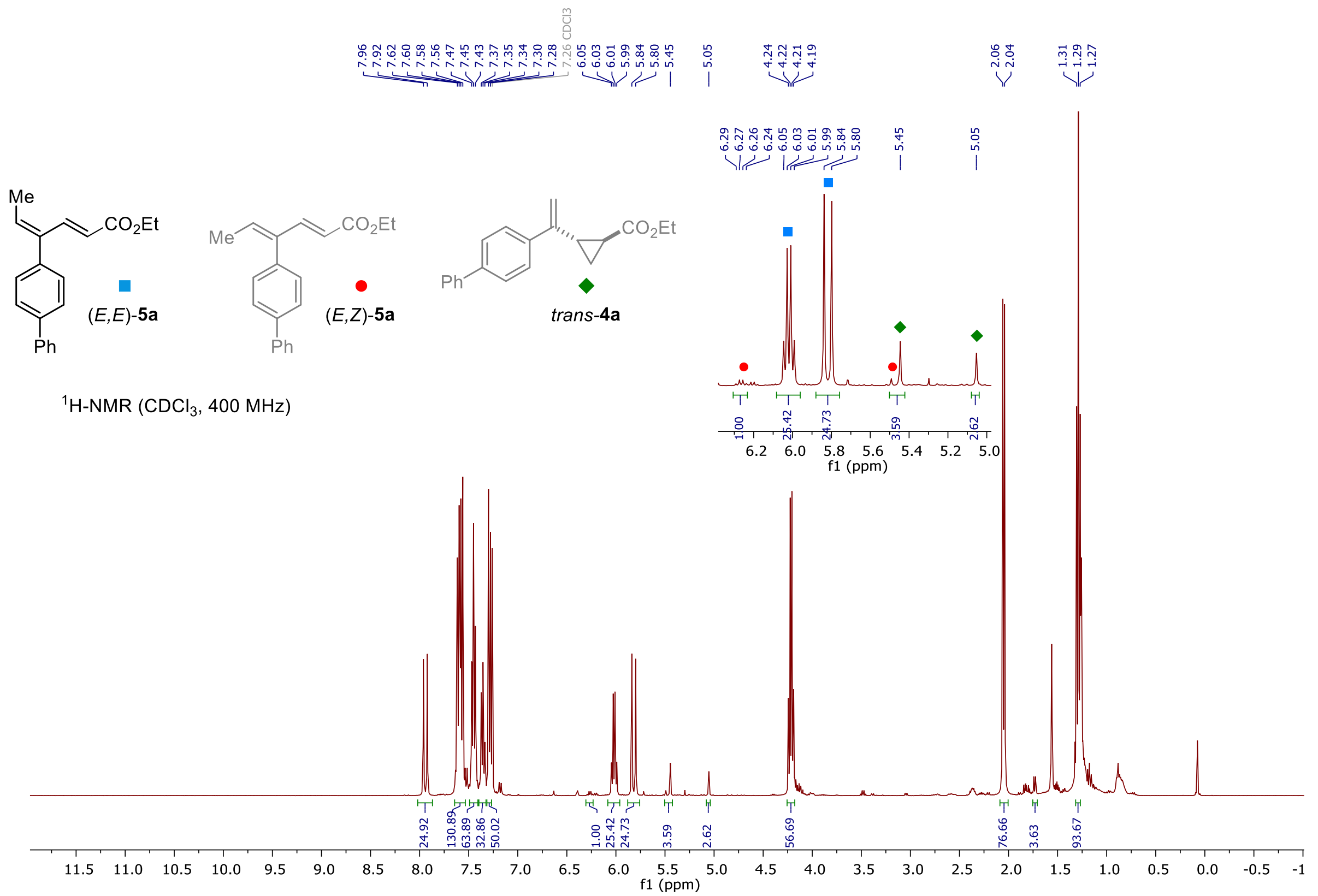



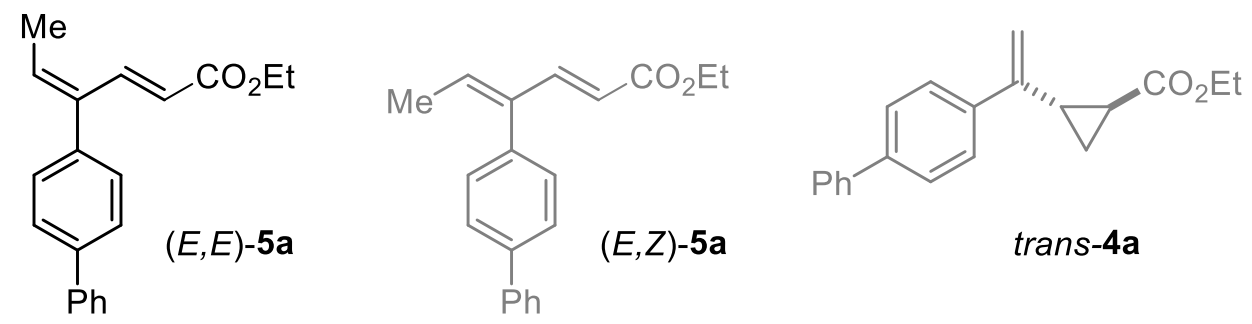

${ }^{13} \mathrm{C}\left\{{ }^{1} \mathrm{H}\right\}-\mathrm{NMR}\left(\mathrm{CDCl}_{3}, 101 \mathrm{MHz}\right)$

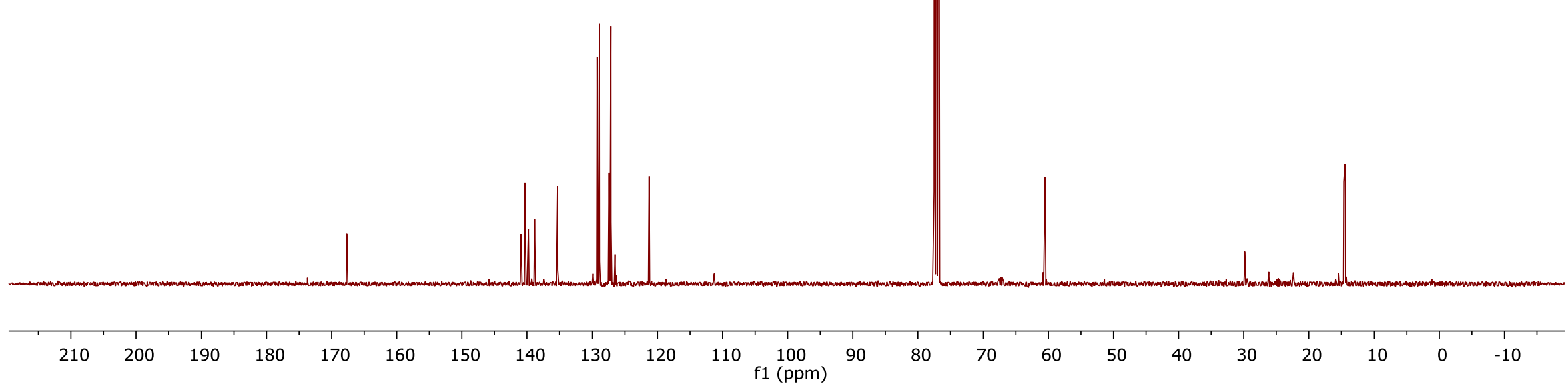




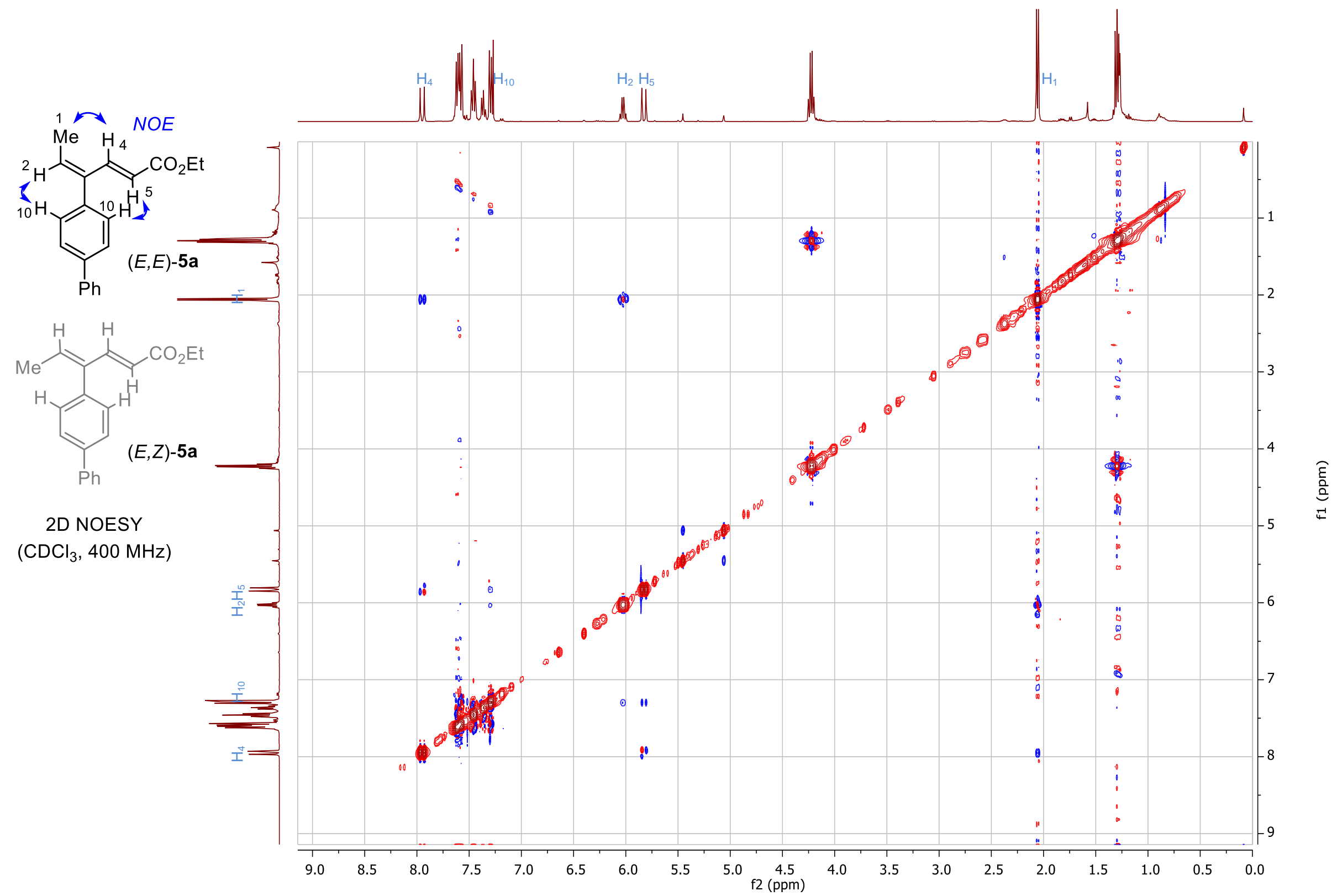



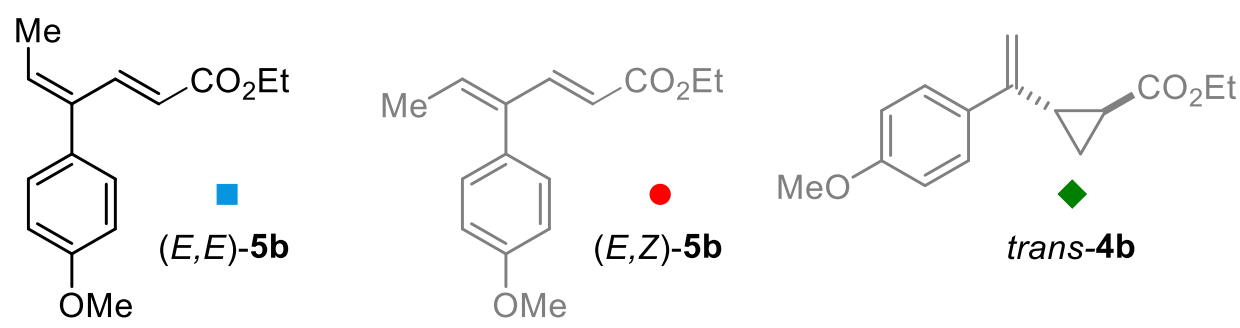

${ }^{1} \mathrm{H}-\mathrm{NMR}\left(\mathrm{CDCl}_{3}, 400 \mathrm{MHz}\right)$
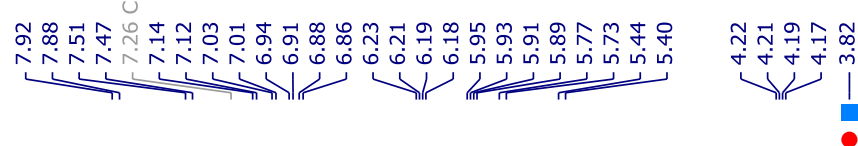

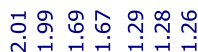

$\mathrm{V} V \mathrm{~V}$

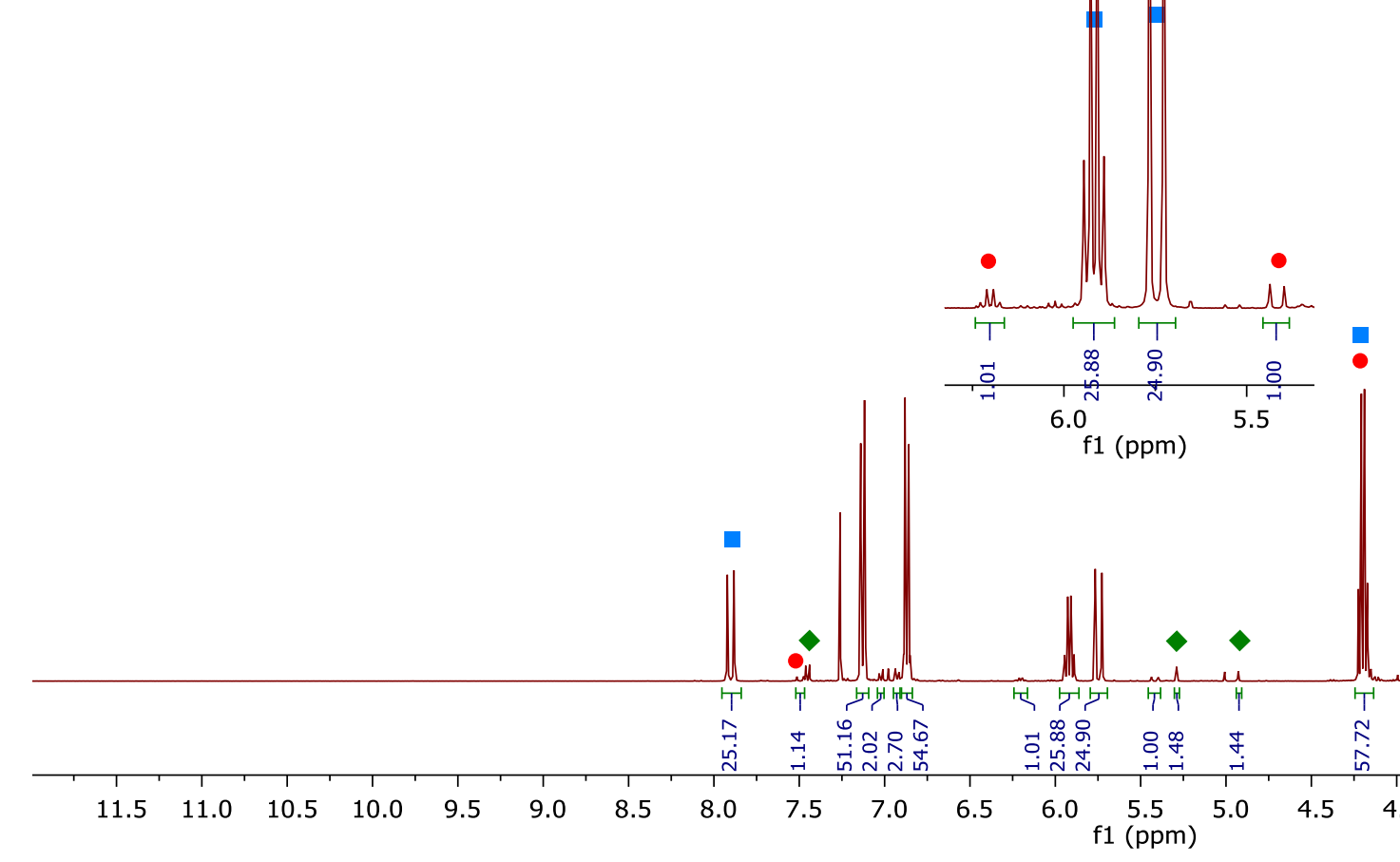

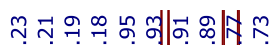
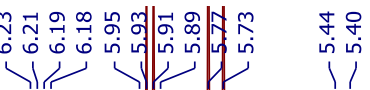

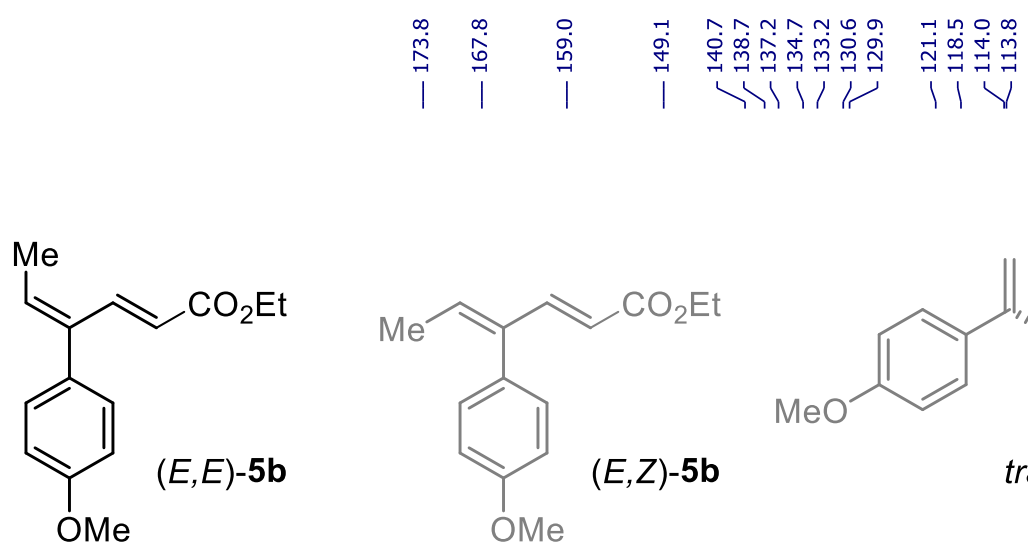

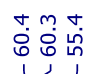

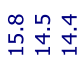

$\checkmark 1$

iV

${ }^{13} \mathrm{C}\left\{{ }^{1} \mathrm{H}\right\}-\mathrm{NMR}\left(\mathrm{CDCl}_{3}, 101 \mathrm{MHz}\right)$

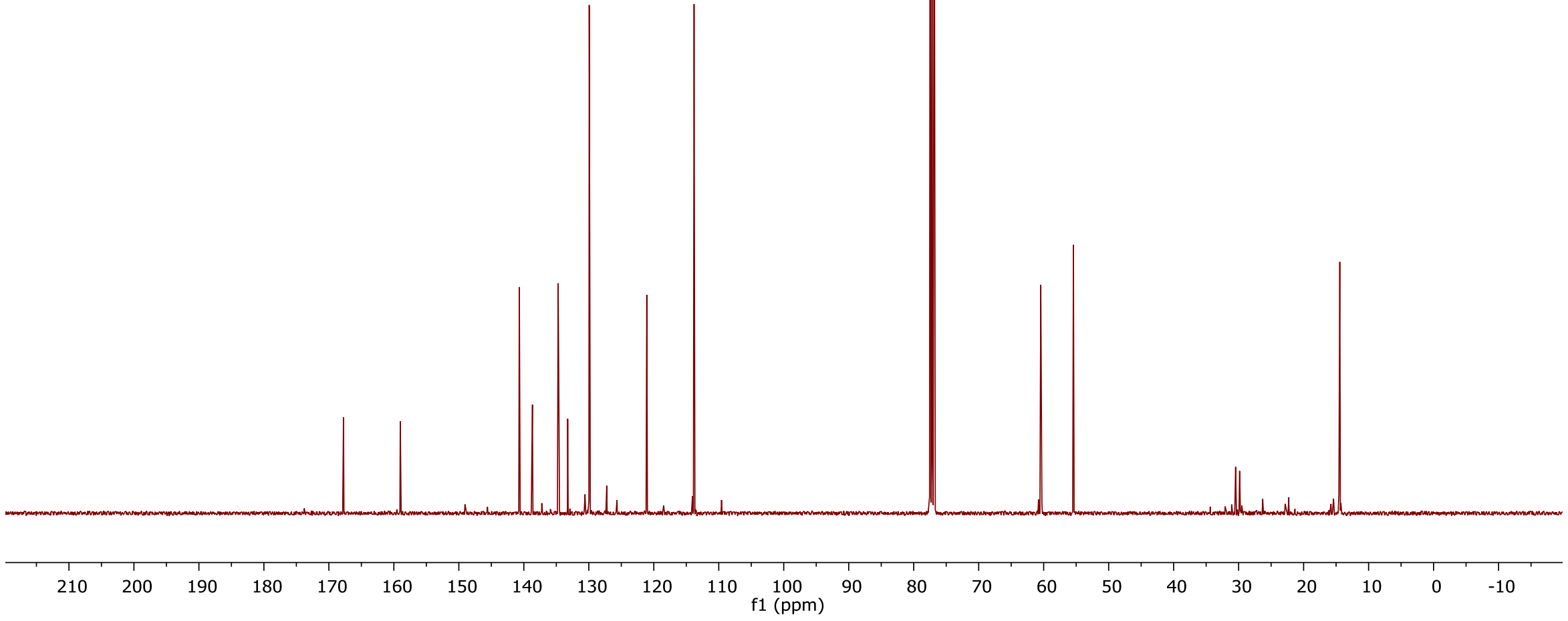




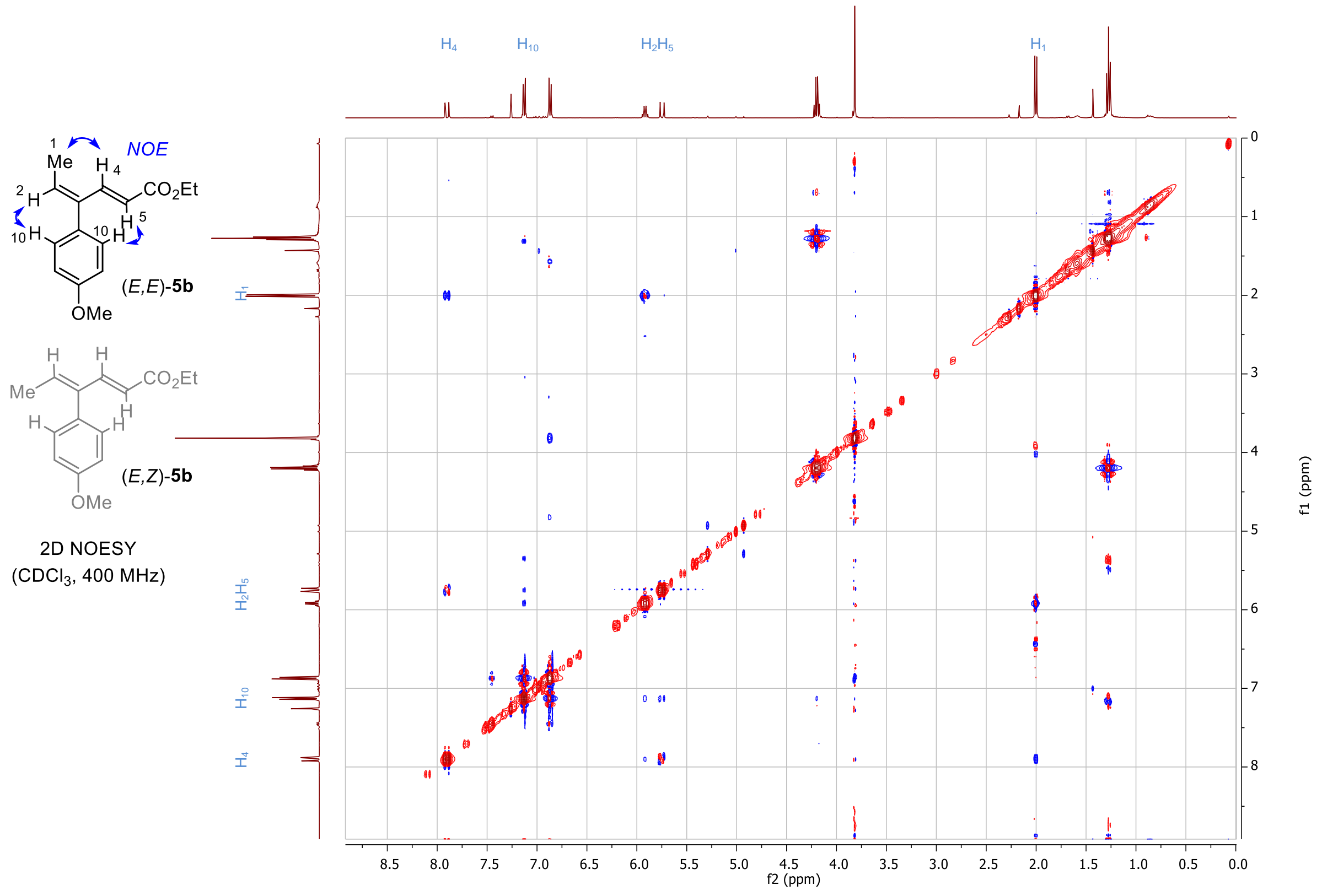




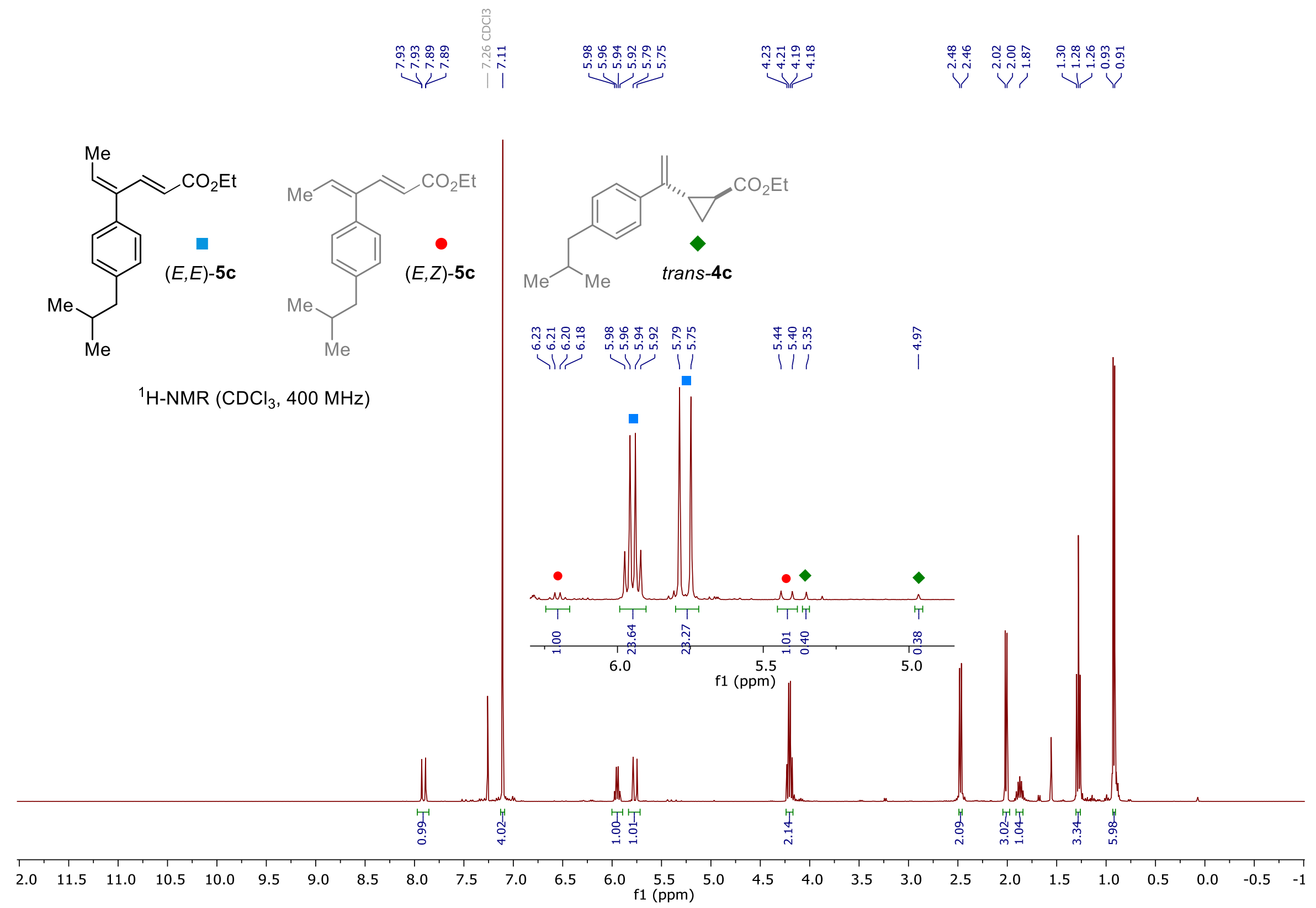



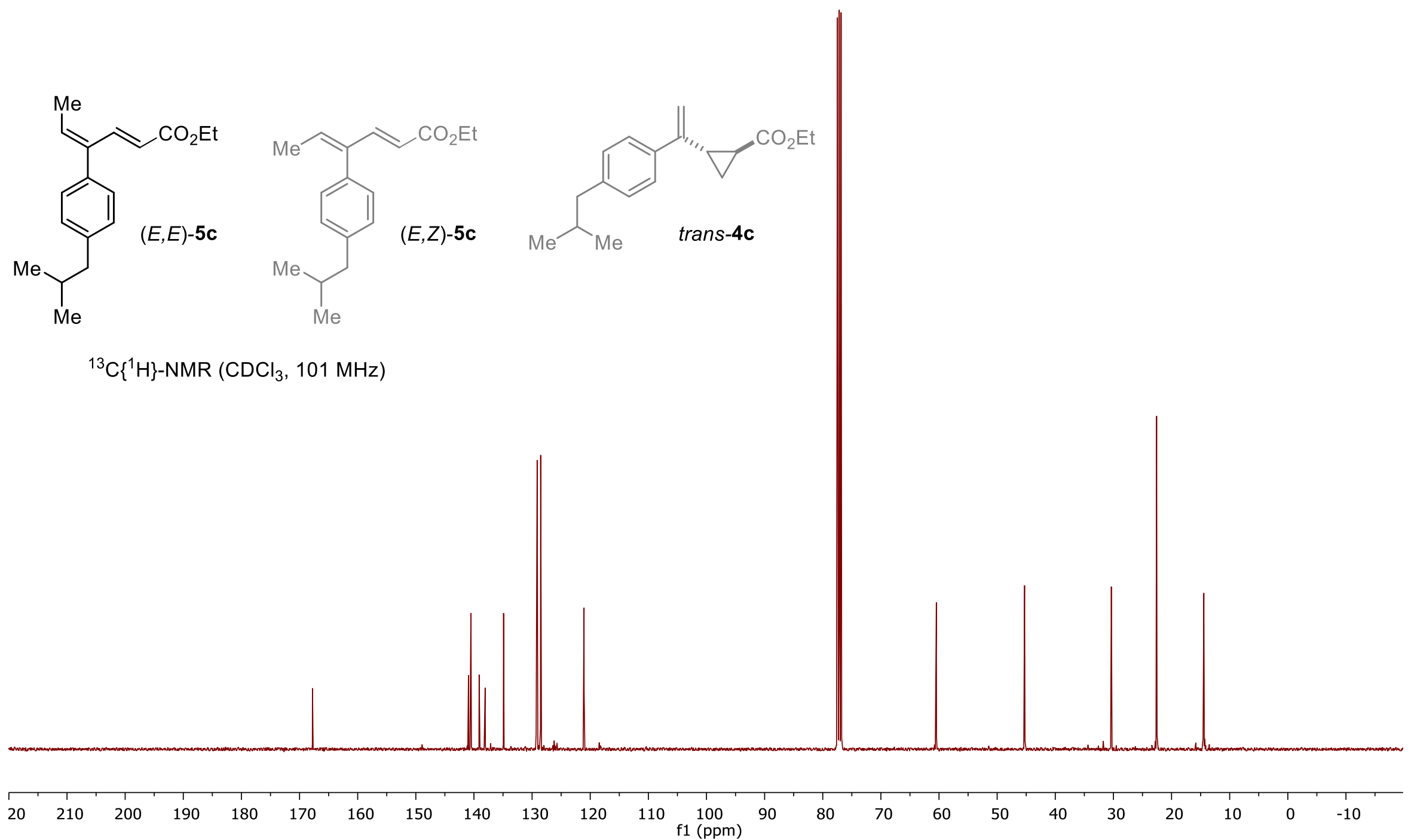

${ }^{13} \mathrm{C}\left\{{ }^{1} \mathrm{H}\right\}-\mathrm{NMR}\left(\mathrm{CDCl}_{3}, 101 \mathrm{MHz}\right)$

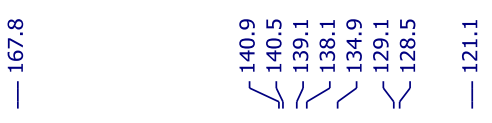

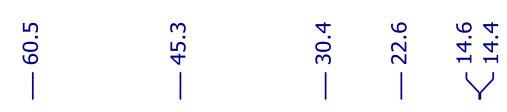




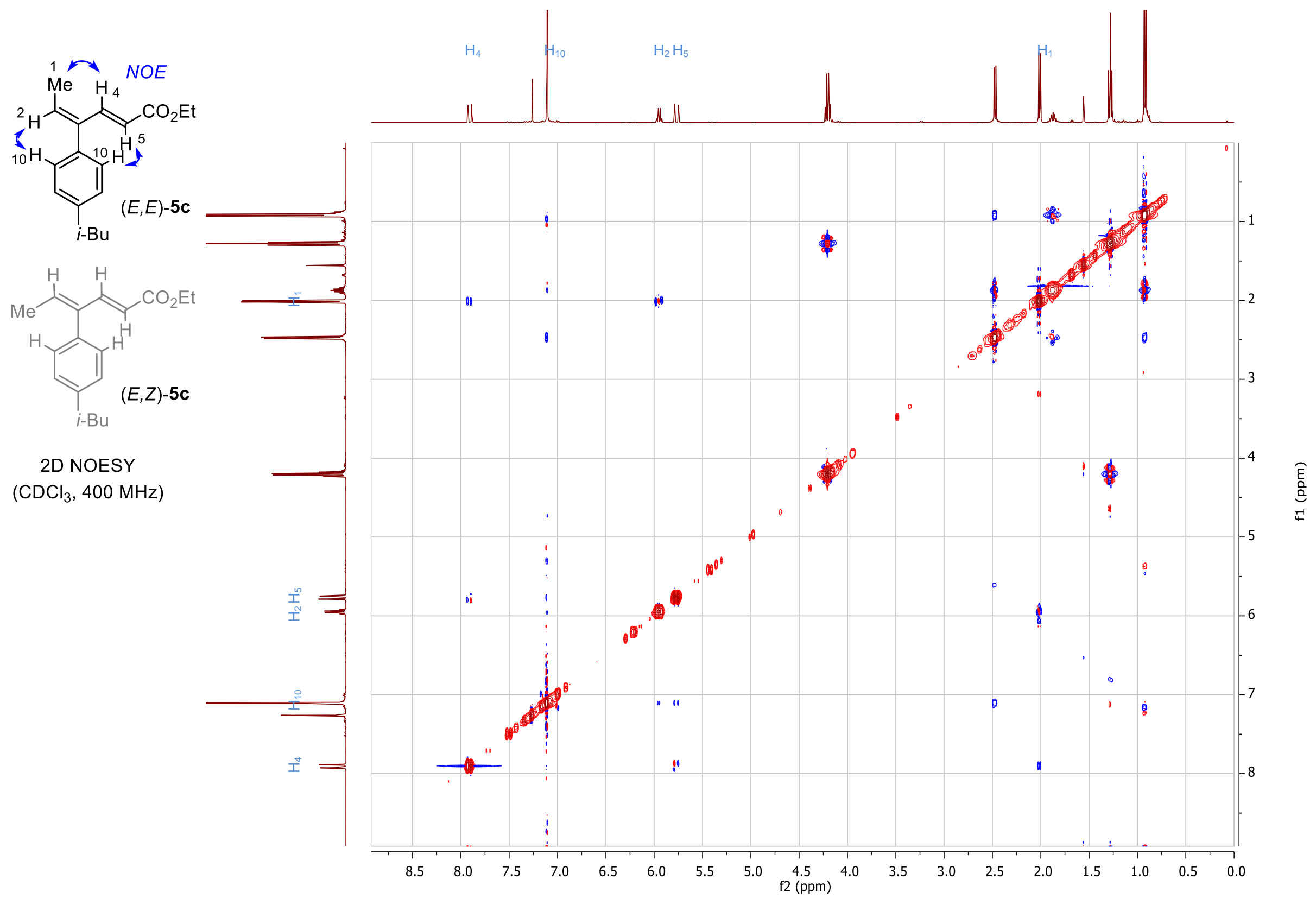


నूณ

ivivit

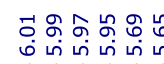

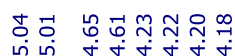

)

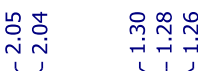
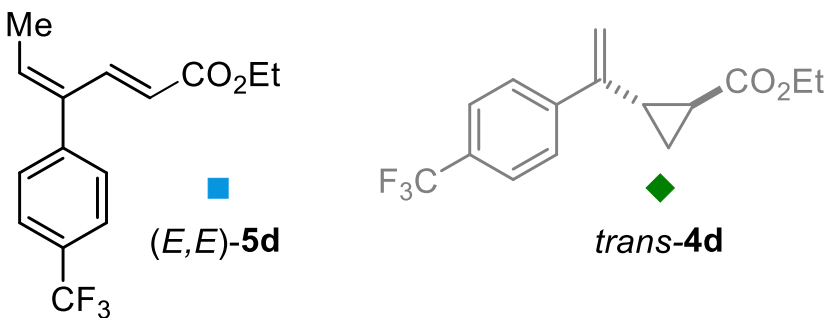

${ }^{1} \mathrm{H}-\mathrm{NMR}\left(\mathrm{CDCl}_{3}, 400 \mathrm{MHz}\right)$

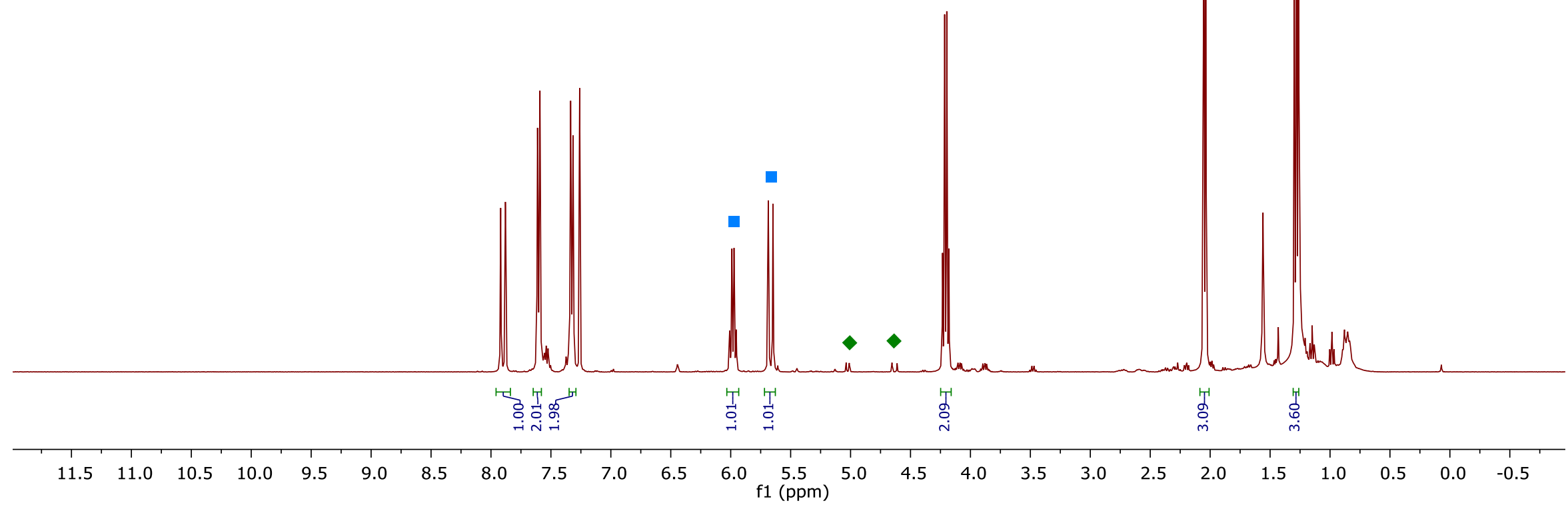




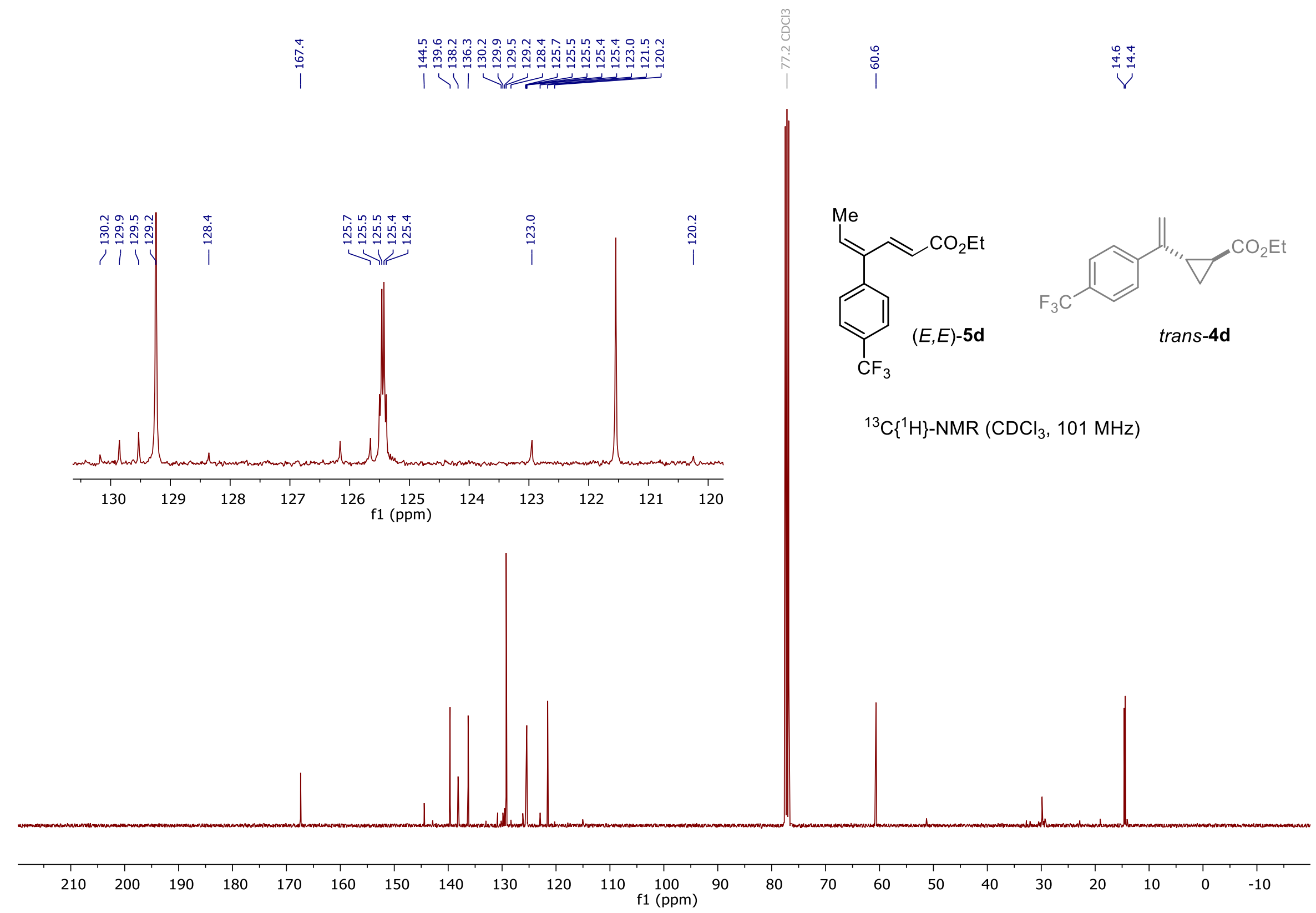




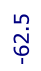

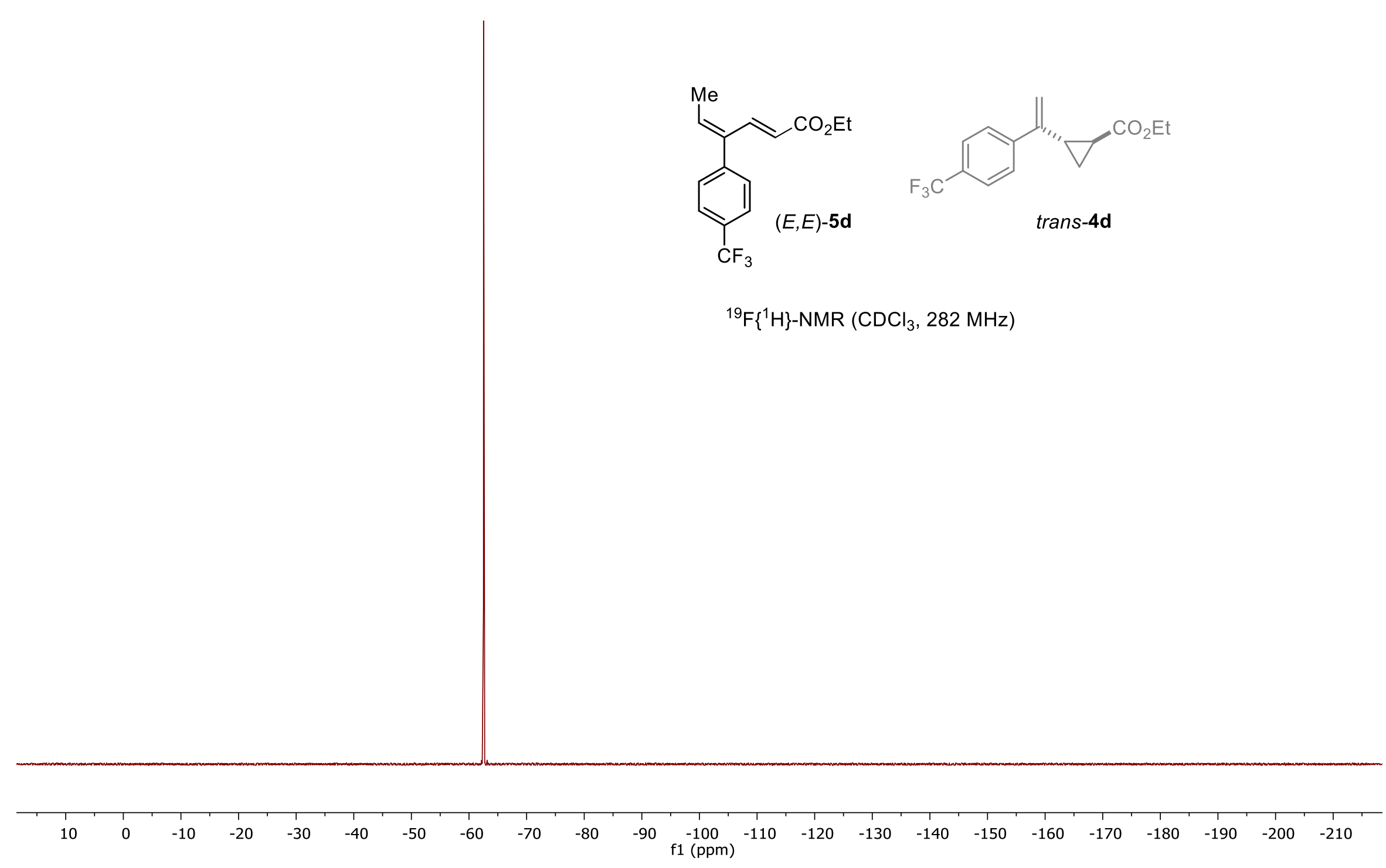


Supporting Information

S40

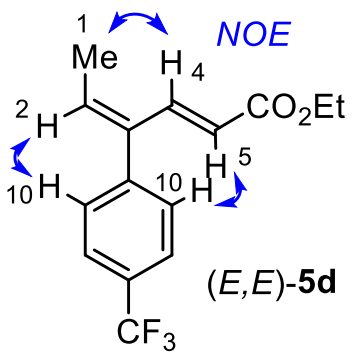

$$
\begin{gathered}
\text { 2D NOESY } \\
\left(\mathrm{CDCl}_{3}, 400 \mathrm{MHz}\right)
\end{gathered}
$$

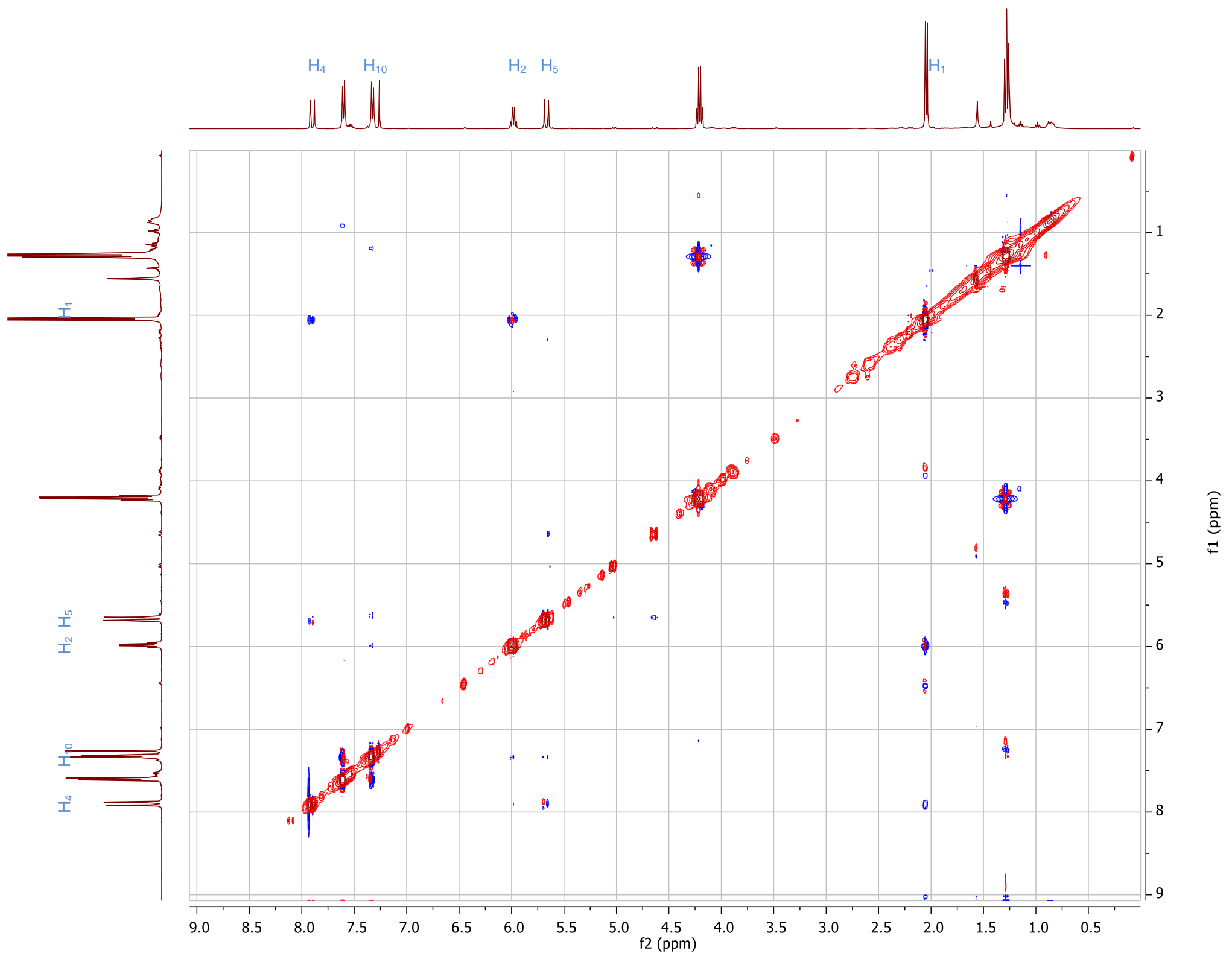




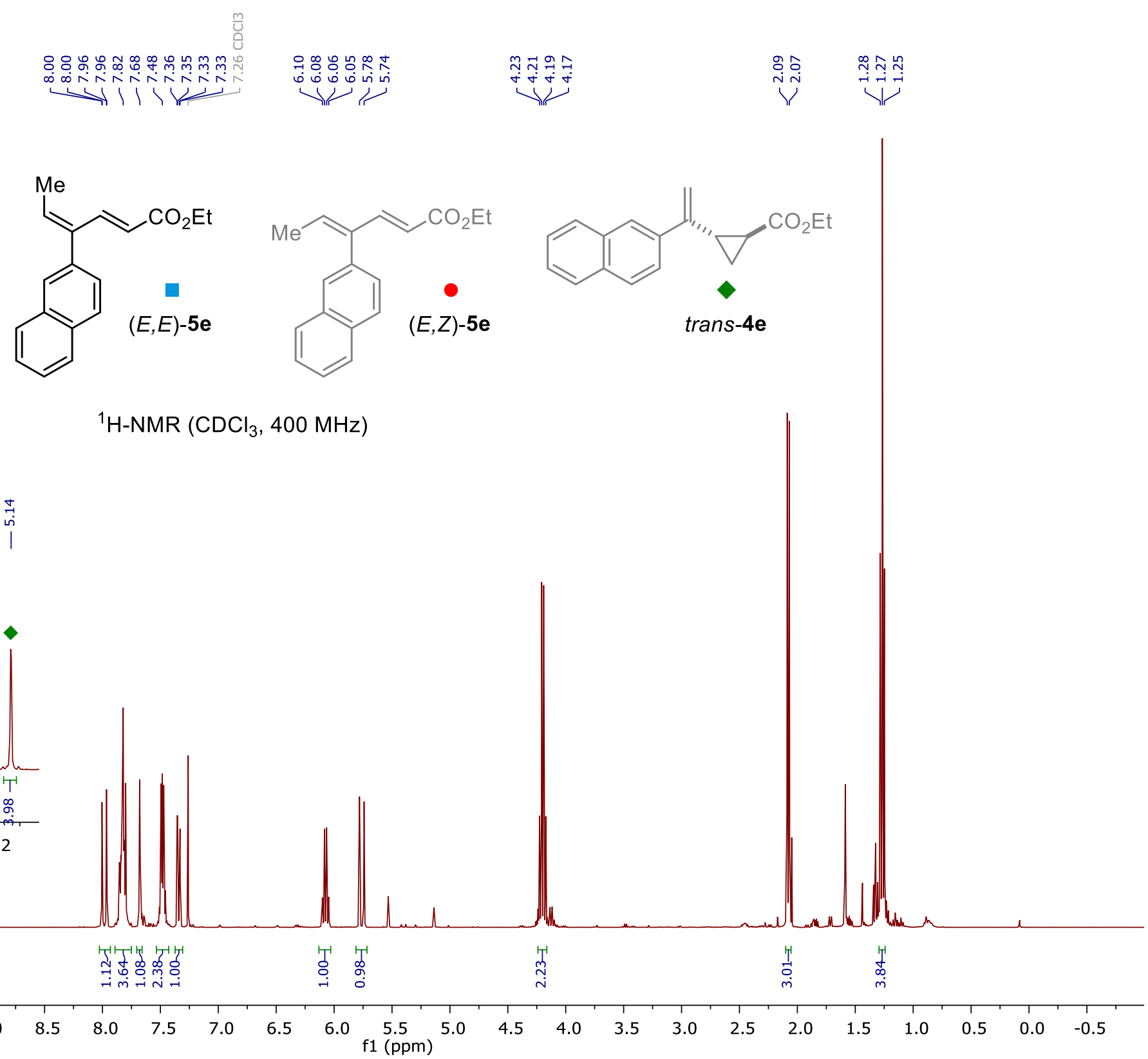



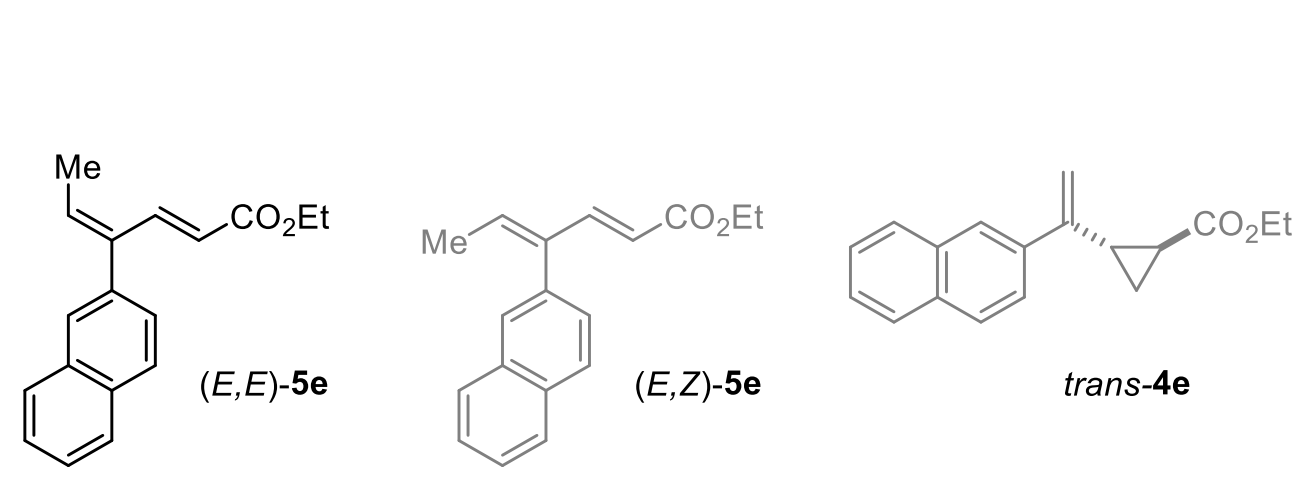

${ }^{13} \mathrm{C}\left\{{ }^{1} \mathrm{H}\right\}-\mathrm{NMR}\left(\mathrm{CDCl}_{3}, 101 \mathrm{MHz}\right)$

$\stackrel{n}{0}$

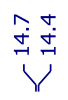

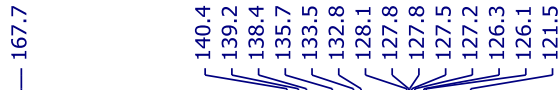

(

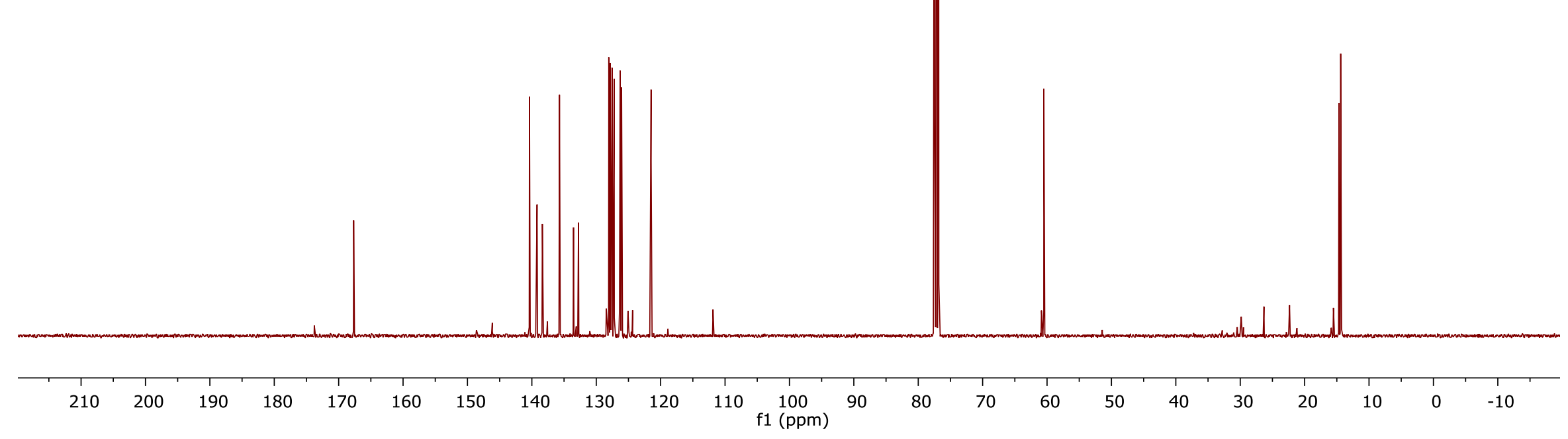



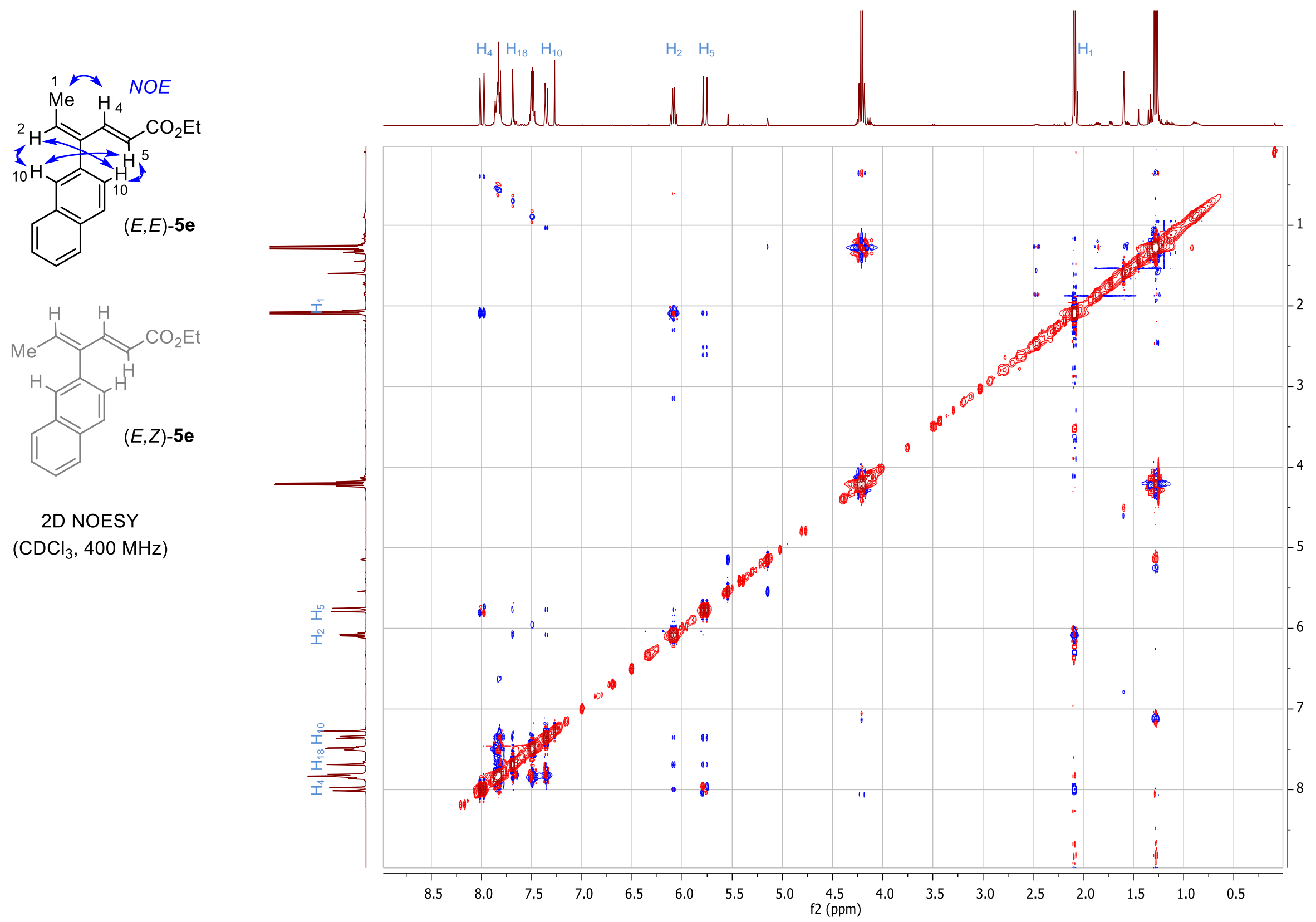


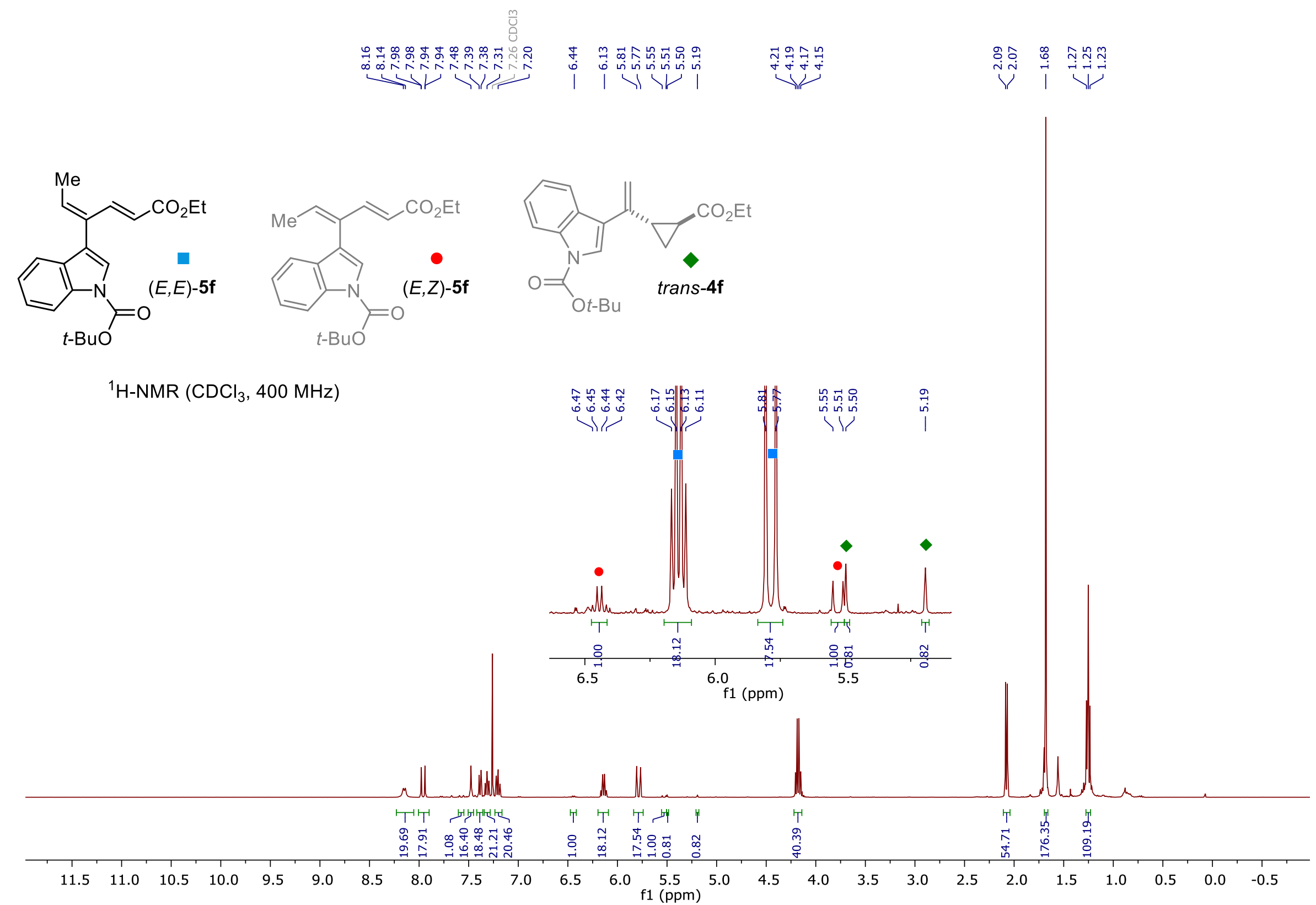



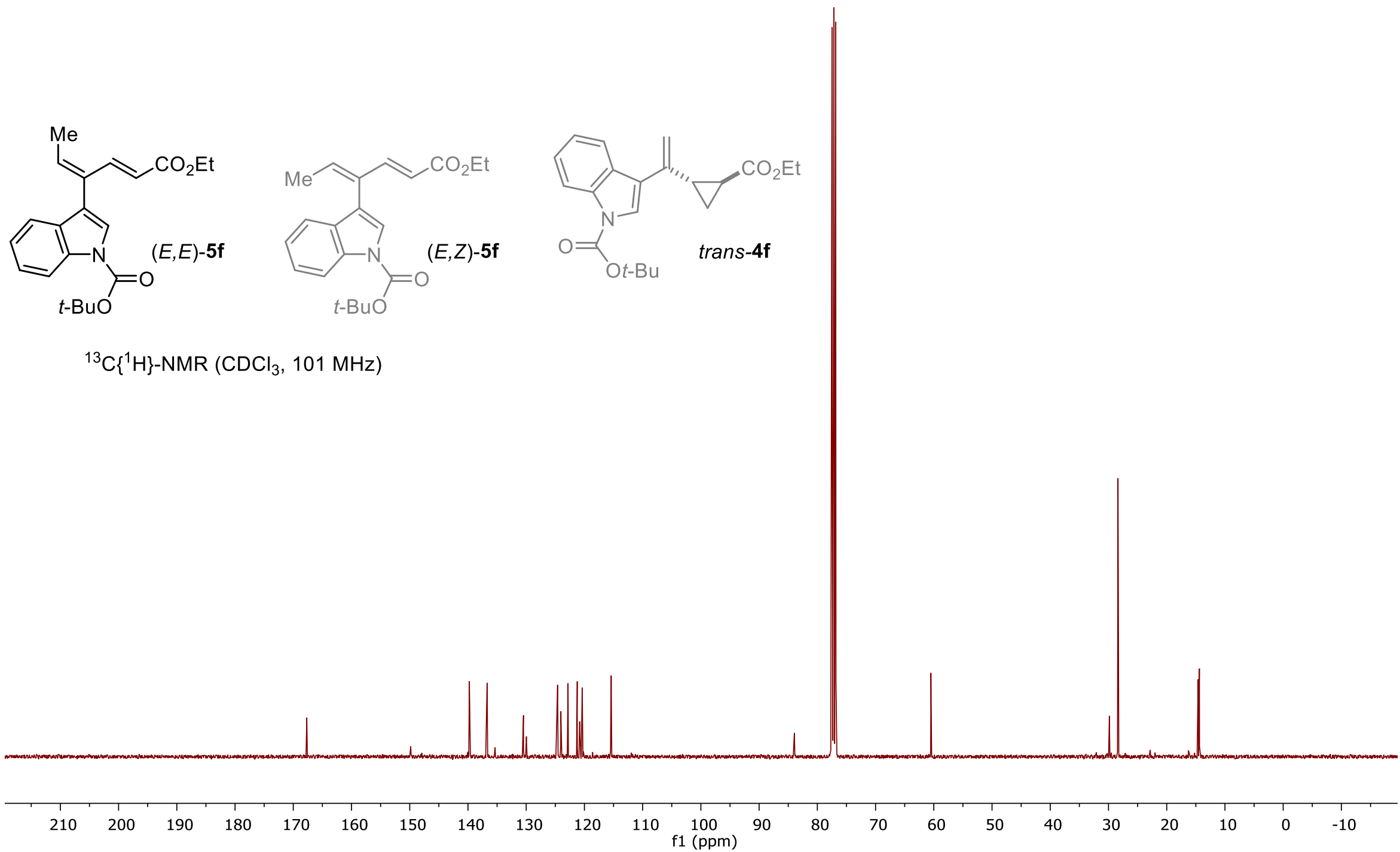

${ }^{13} \mathrm{C}\left\{{ }^{1} \mathrm{H}\right\}-\mathrm{NMR}\left(\mathrm{CDCl}_{3}, 101 \mathrm{MHz}\right)$

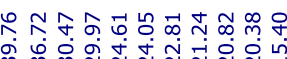

|

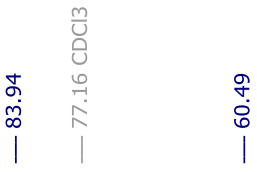

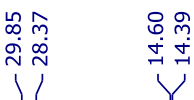




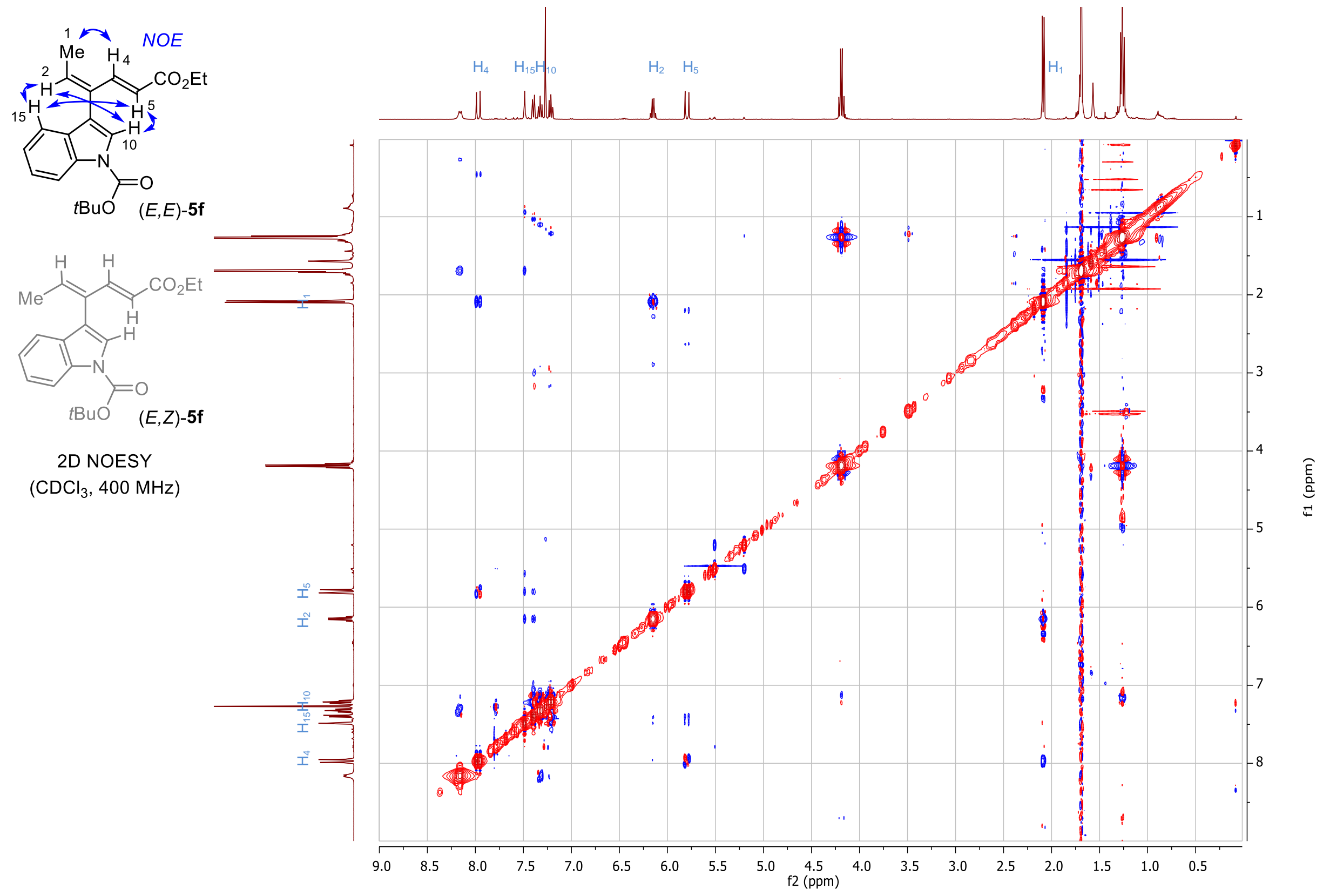




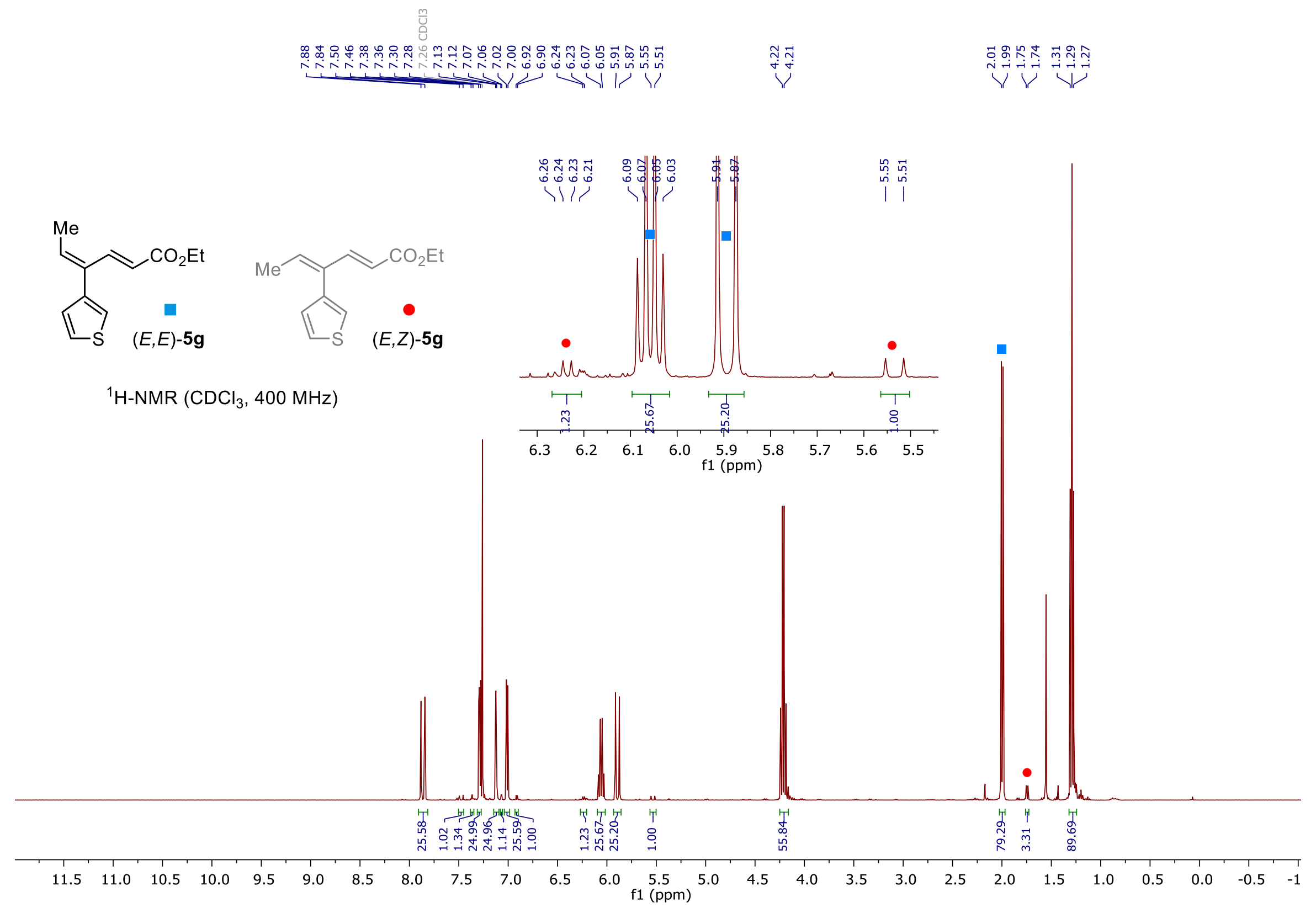




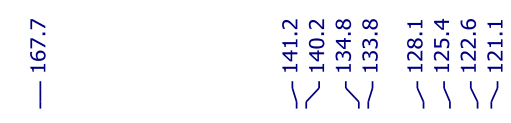

$\stackrel{n}{0}$

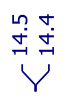
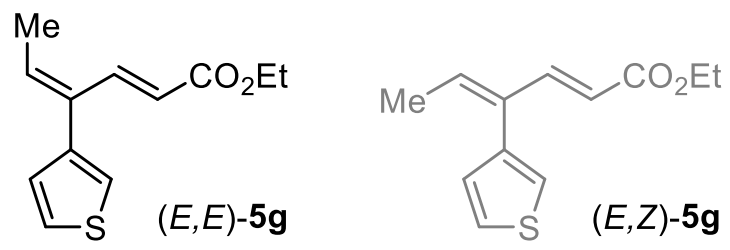

${ }^{13} \mathrm{C}\left\{{ }^{1} \mathrm{H}\right\}-\mathrm{NMR}\left(\mathrm{CDCl}_{3}, 101 \mathrm{MHz}\right)$

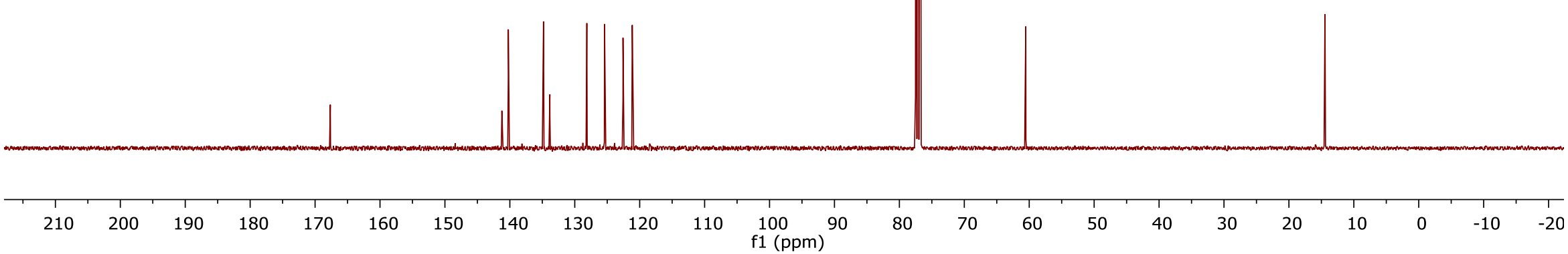




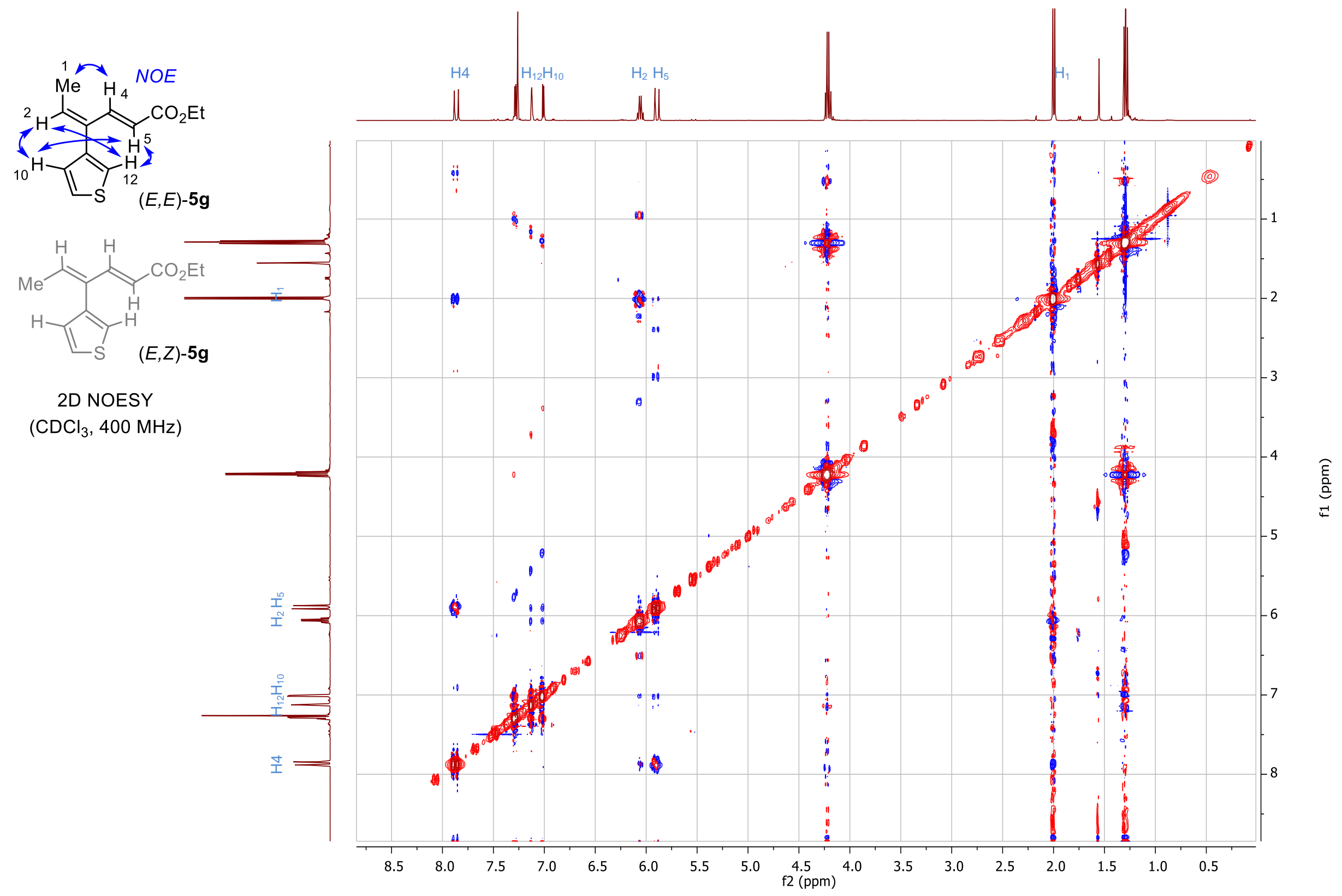




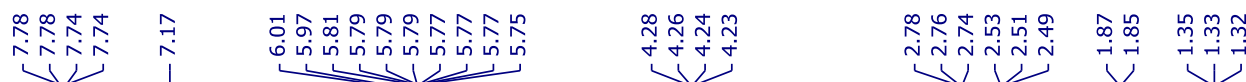

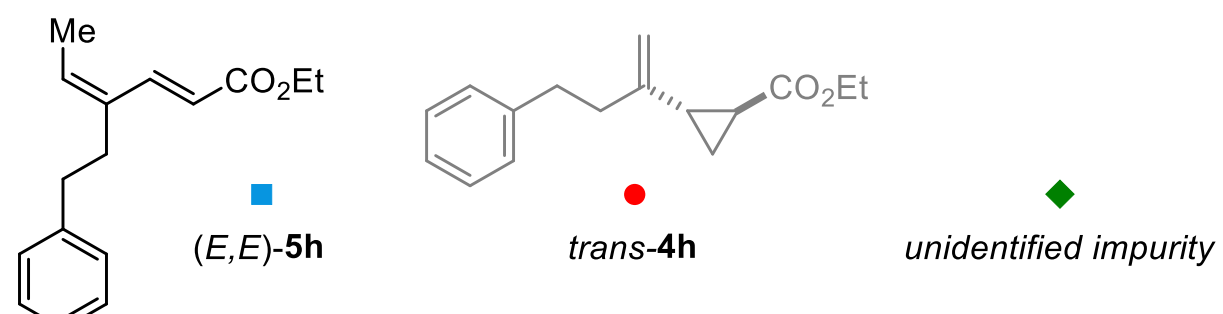

${ }^{1} \mathrm{H}-\mathrm{NMR}\left(\mathrm{CDCl}_{3}, 400 \mathrm{MHz}\right)$

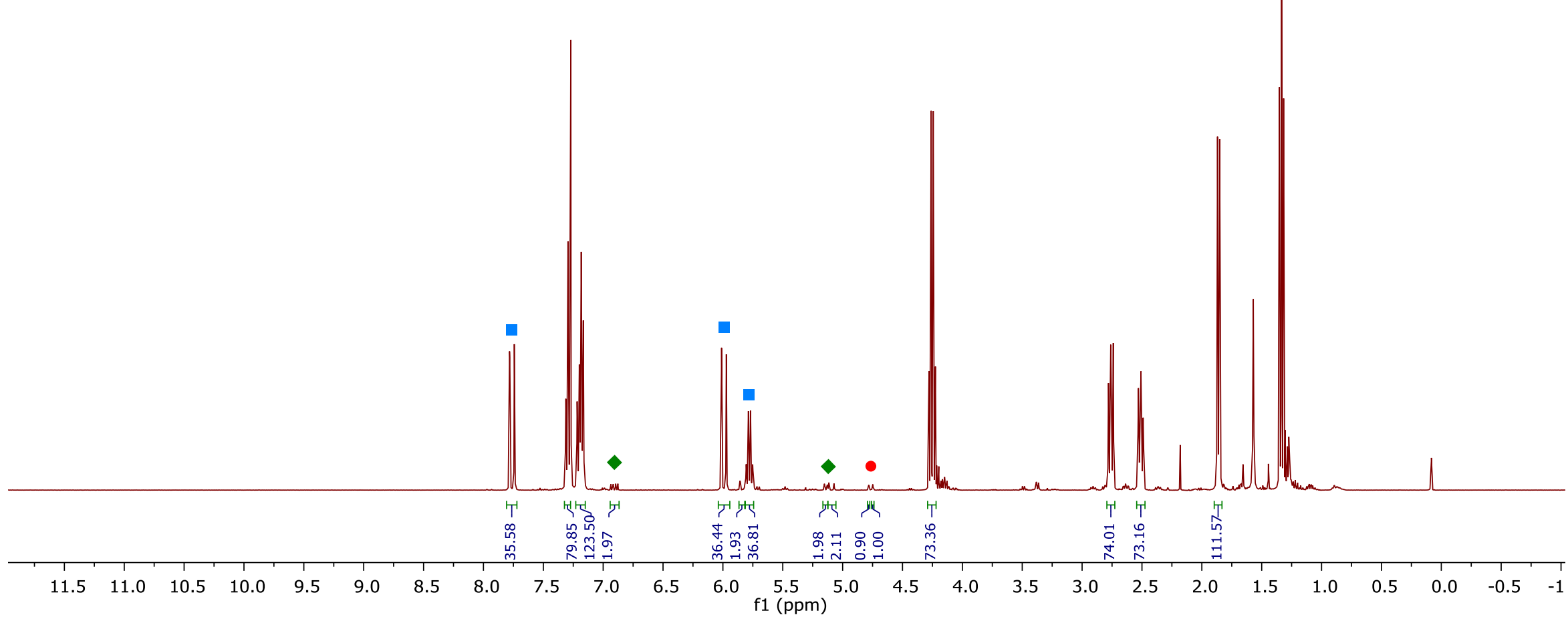




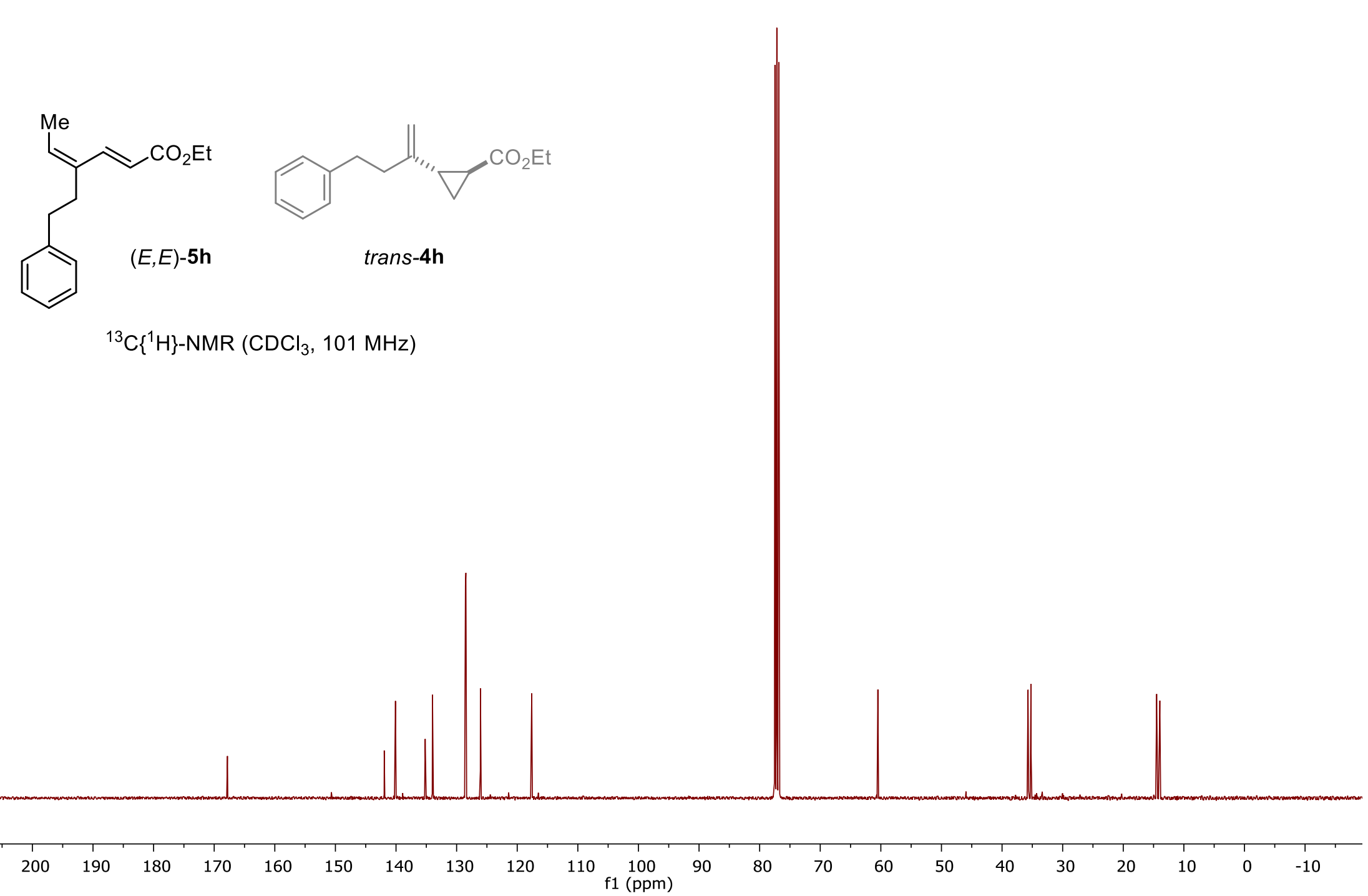

${ }^{13} \mathrm{C}\left\{{ }^{1} \mathrm{H}\right\}-\mathrm{NMR}\left(\mathrm{CDCl}_{3}, 101 \mathrm{MHz}\right)$

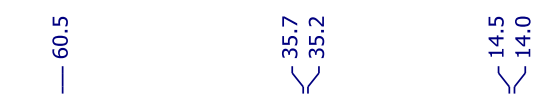




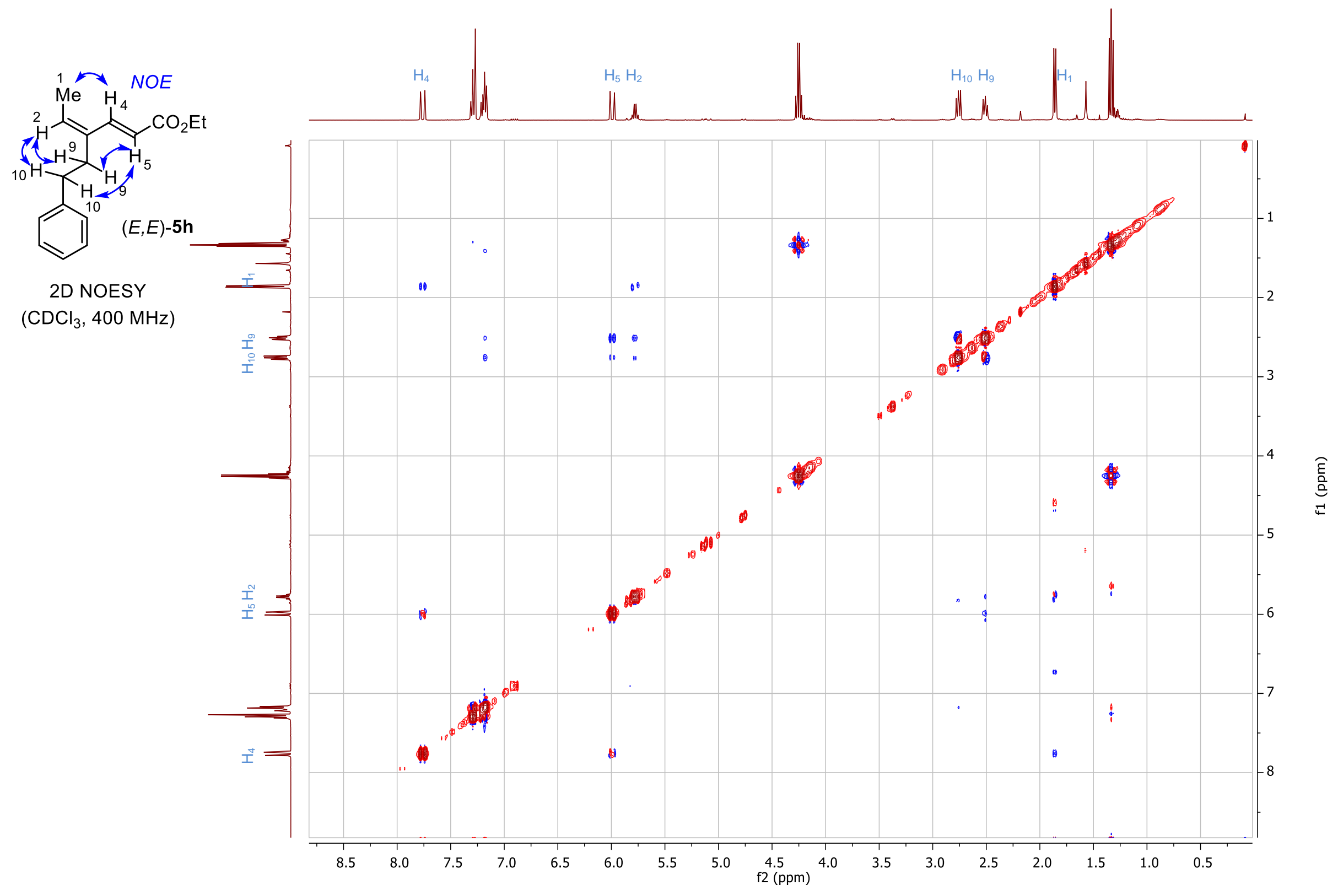




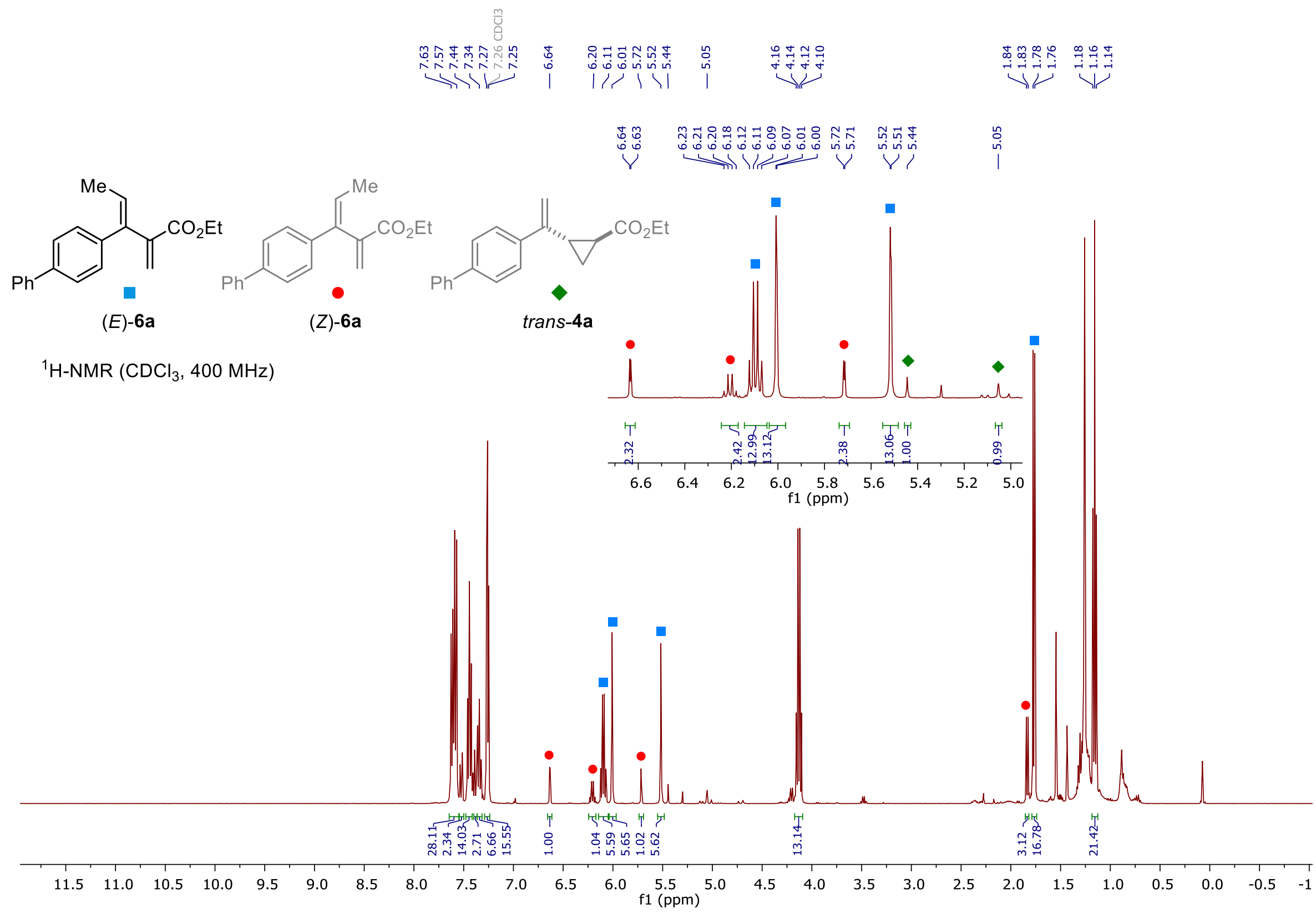




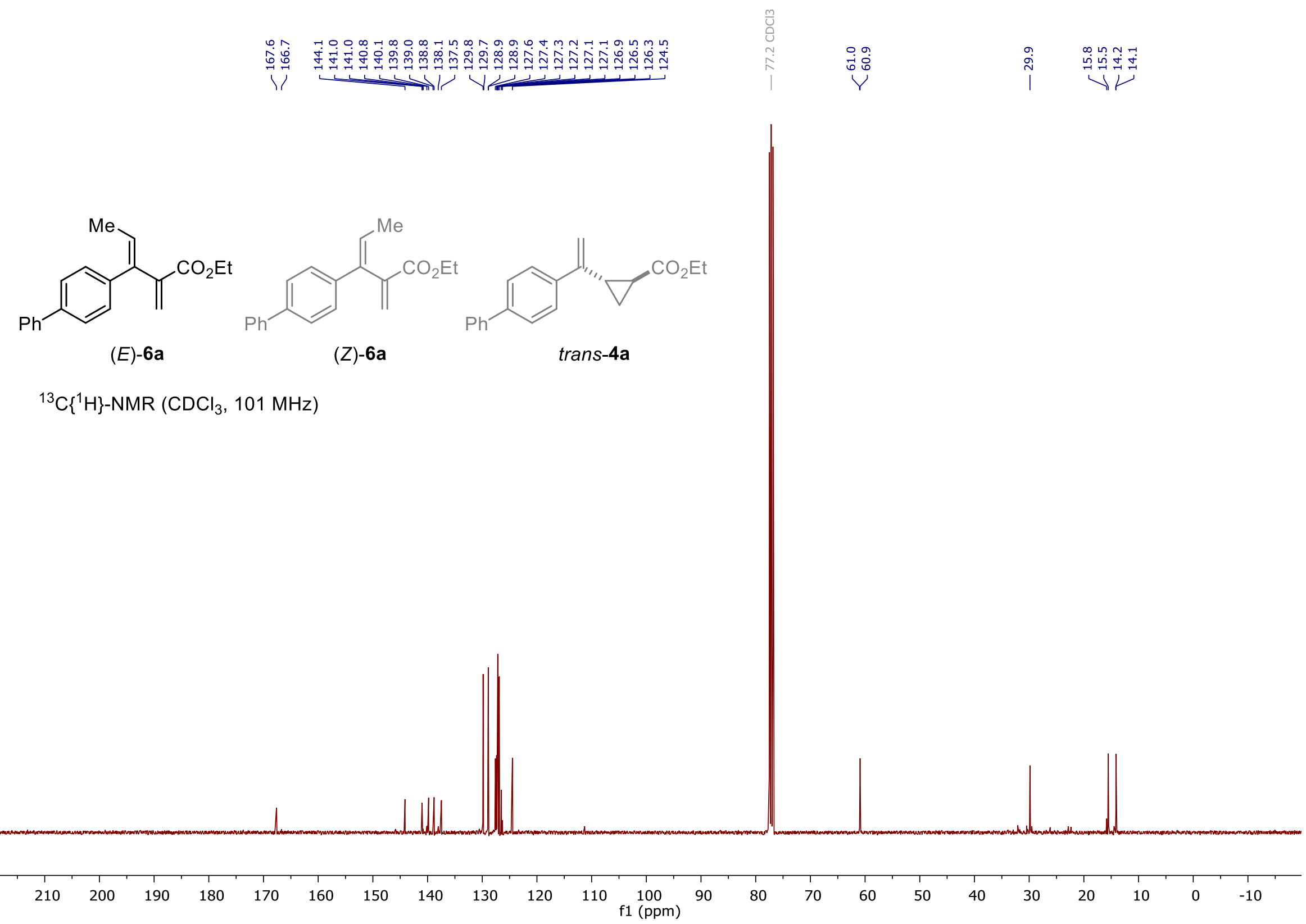




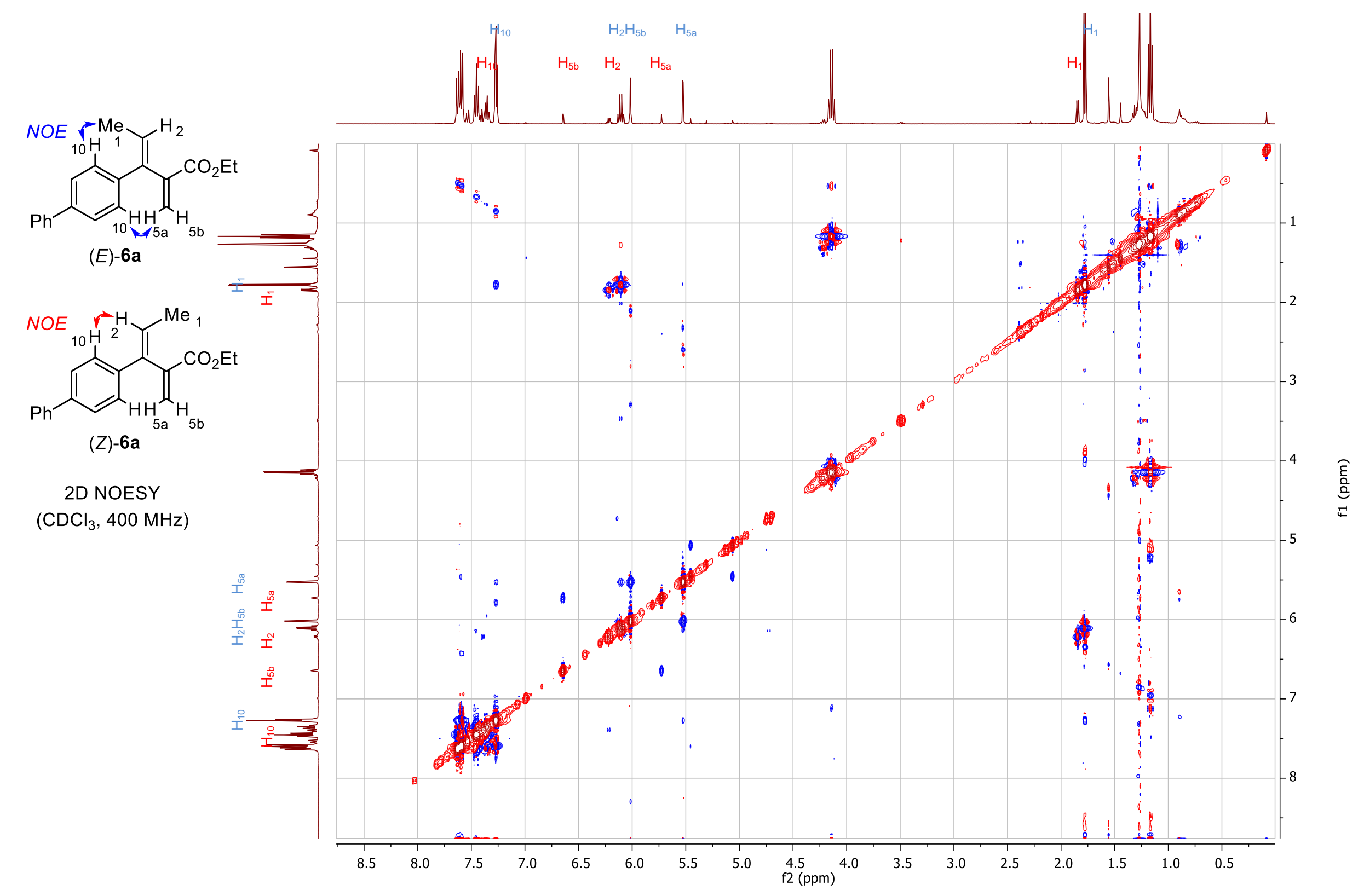




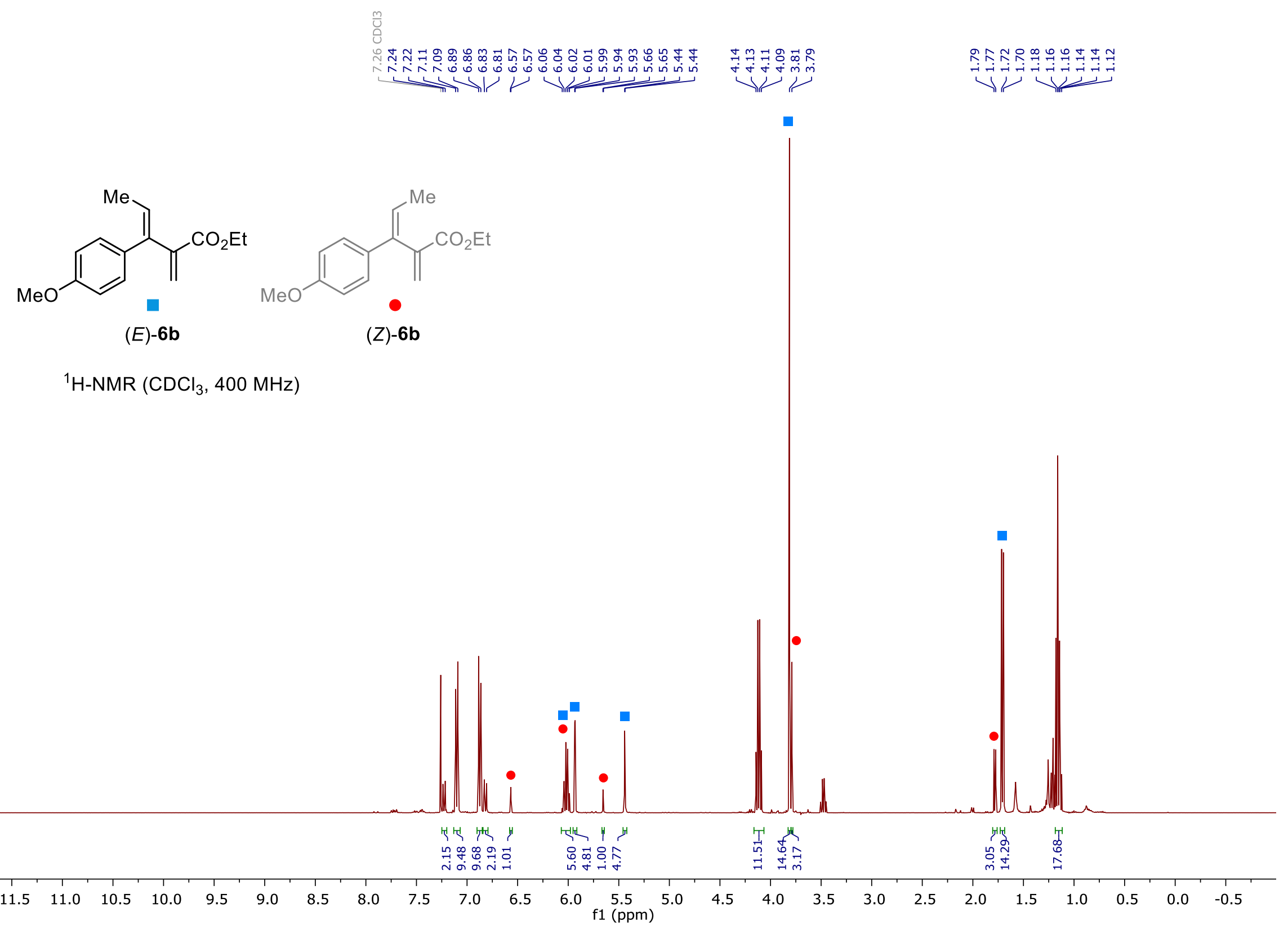




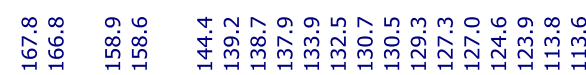

)

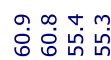

ค่ำ

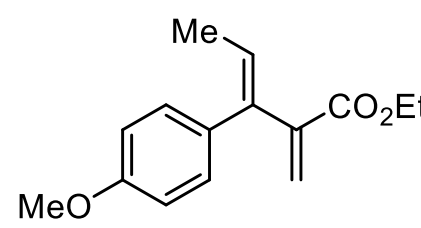

(E)-6b

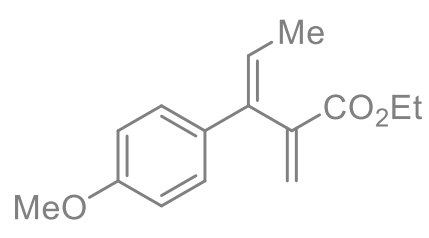

(Z)-6b

${ }^{13} \mathrm{C}\left\{{ }^{1} \mathrm{H}\right\}-\mathrm{NMR}\left(\mathrm{CDCl}_{3}, 101 \mathrm{MHz}\right)$

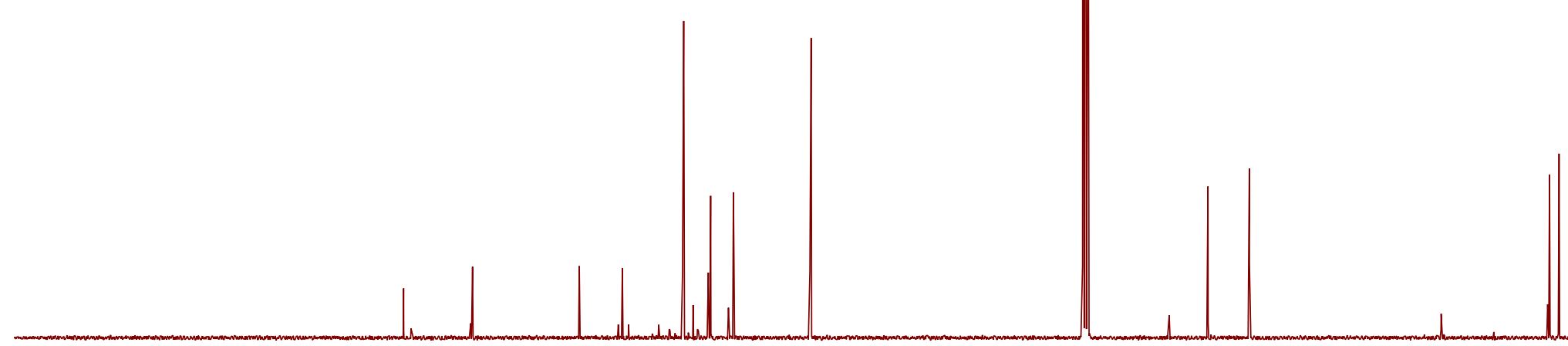

$\begin{array}{llllllllllll}210 & 200 & 190 & 180 & 170 & 160 & 150 & 140 & 130 & 120 & 110 & \begin{array}{c}100 \\ \mathrm{f} 1(\mathrm{ppm})\end{array}\end{array}$

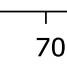



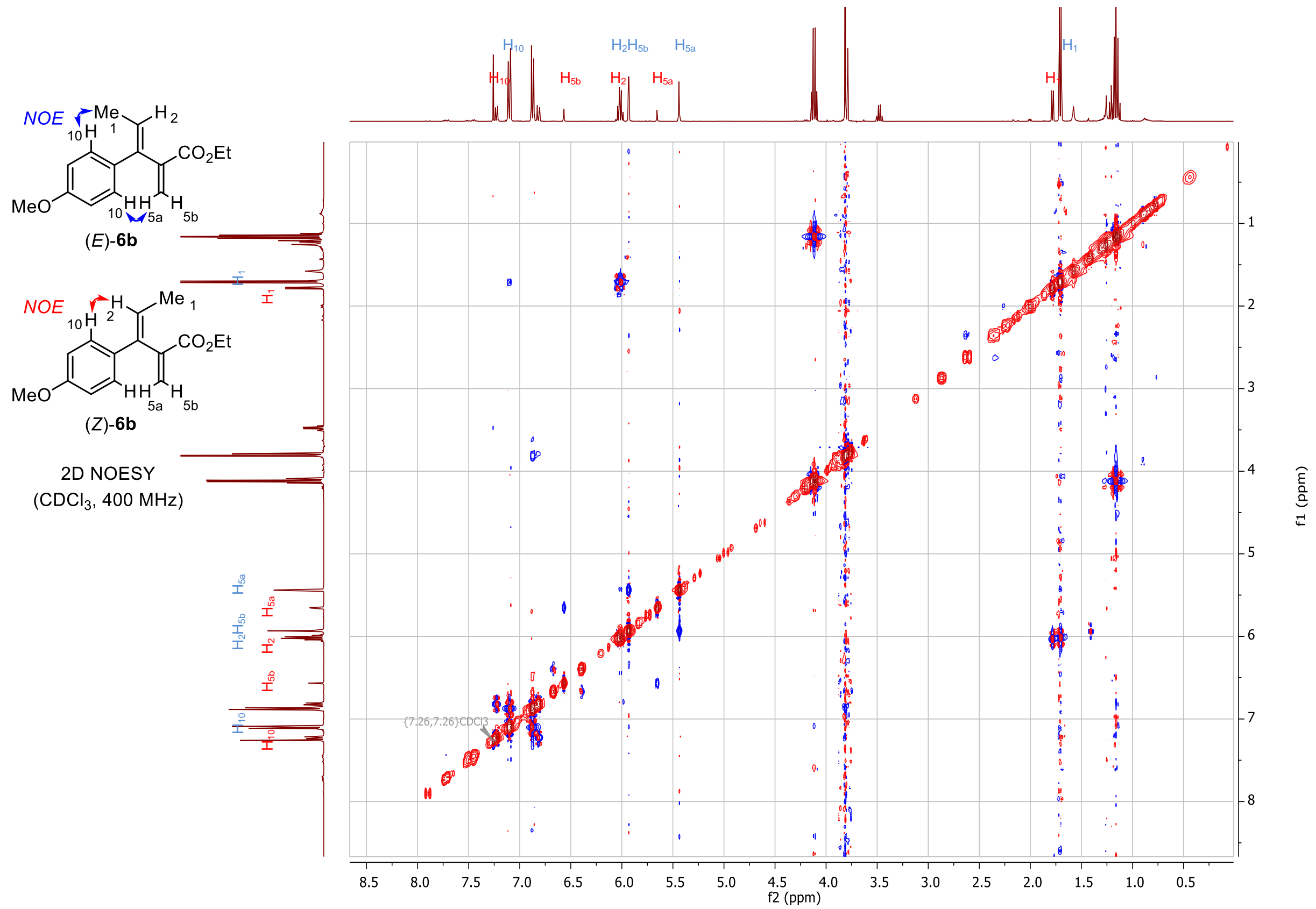


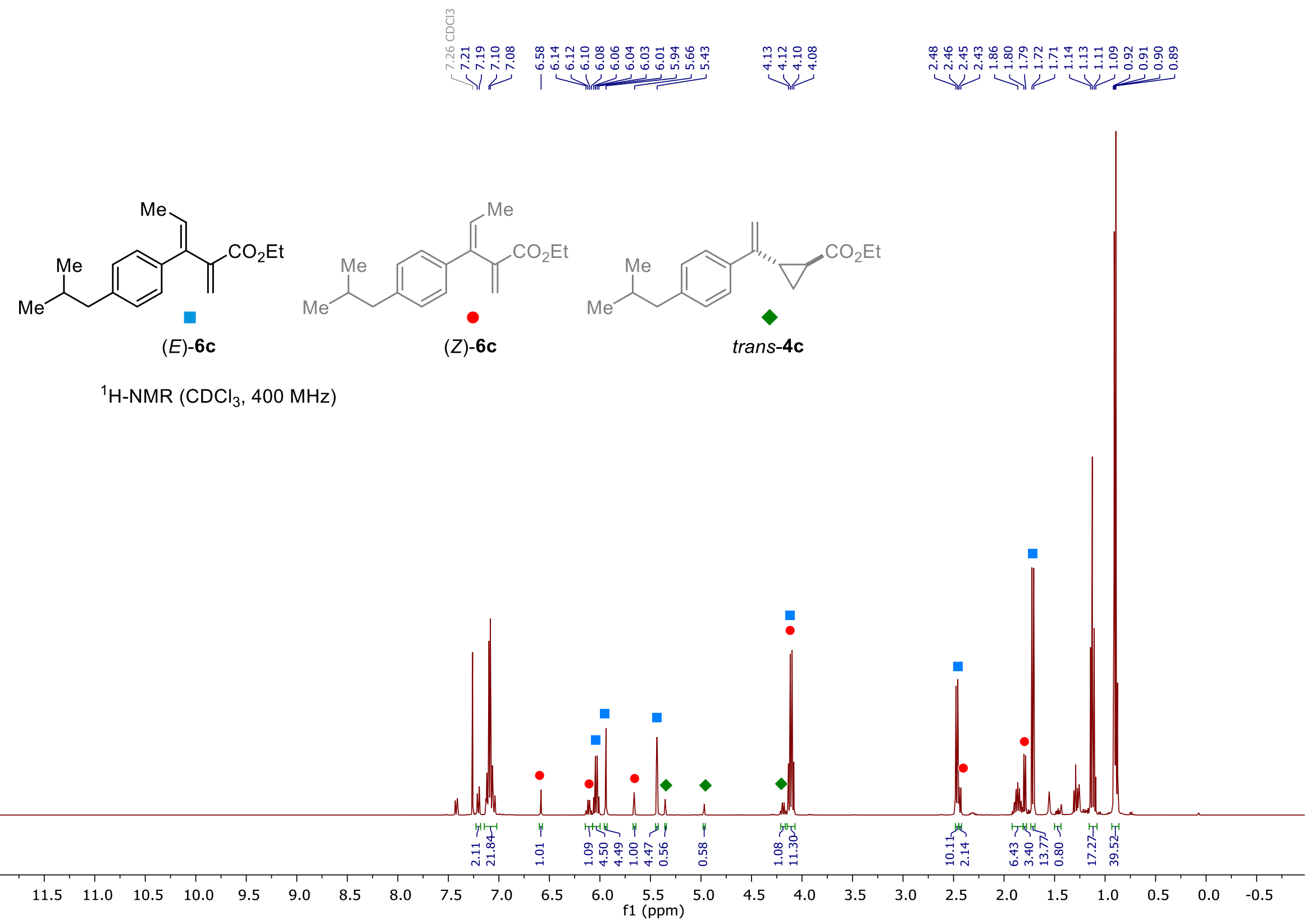




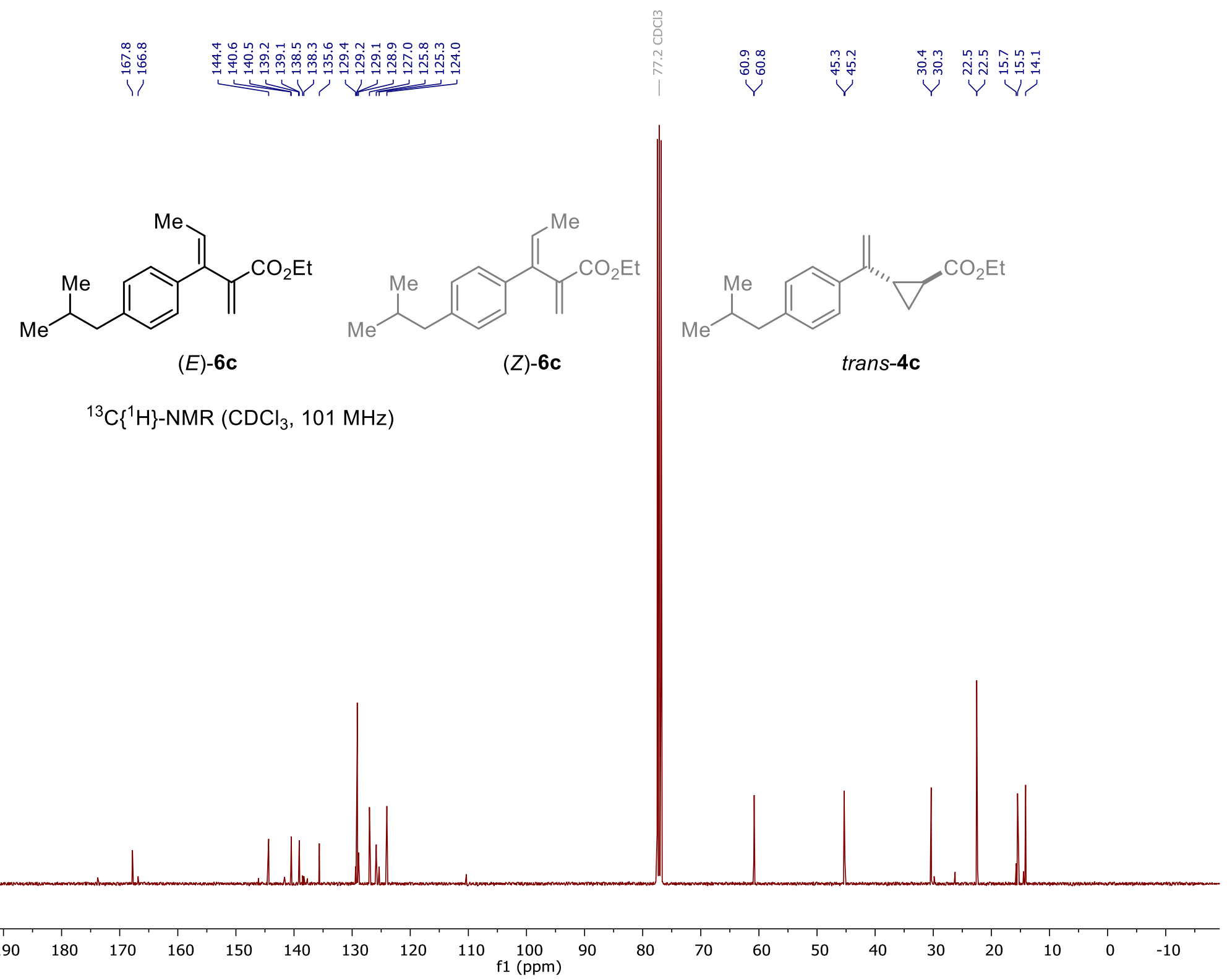




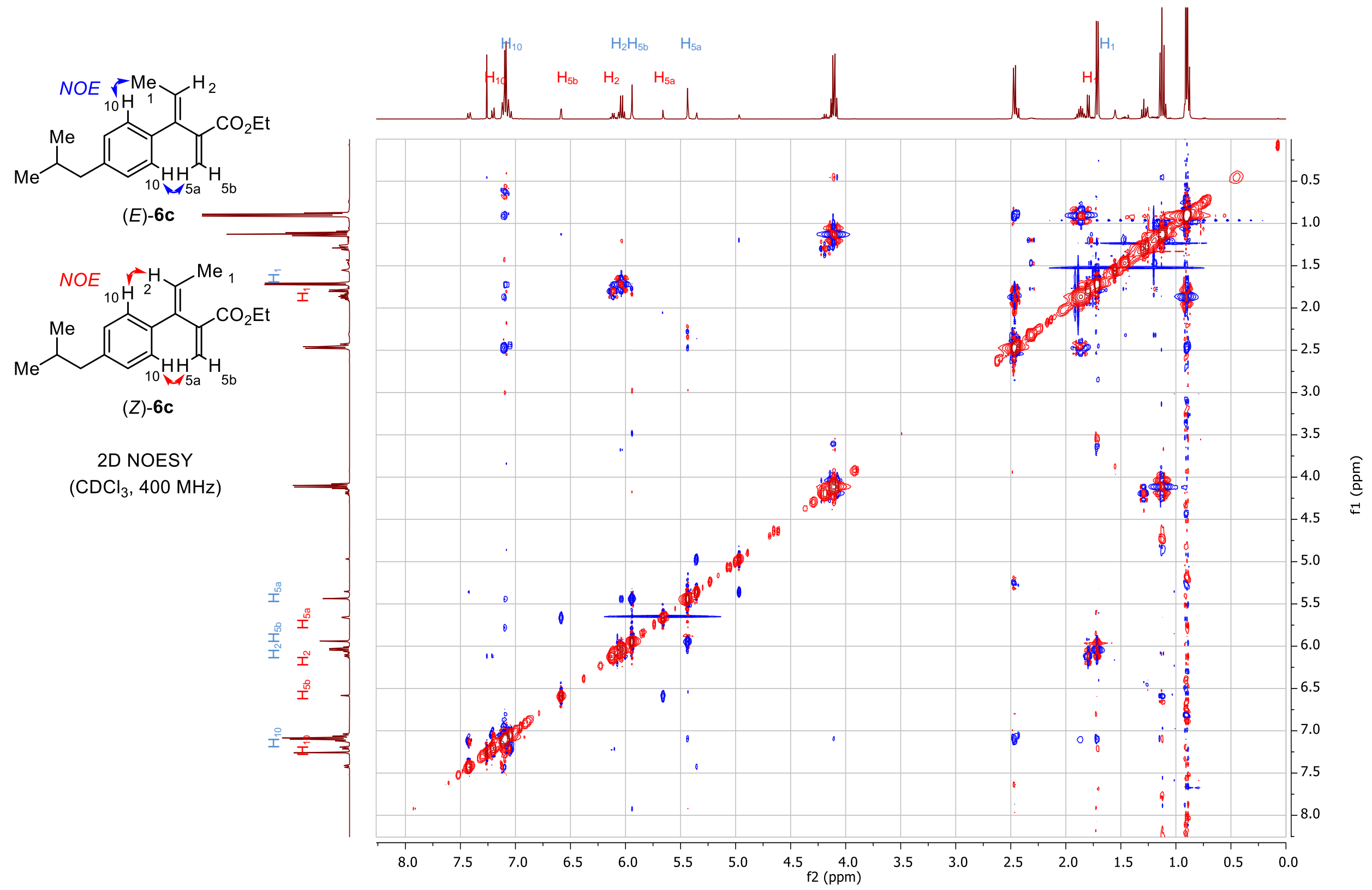




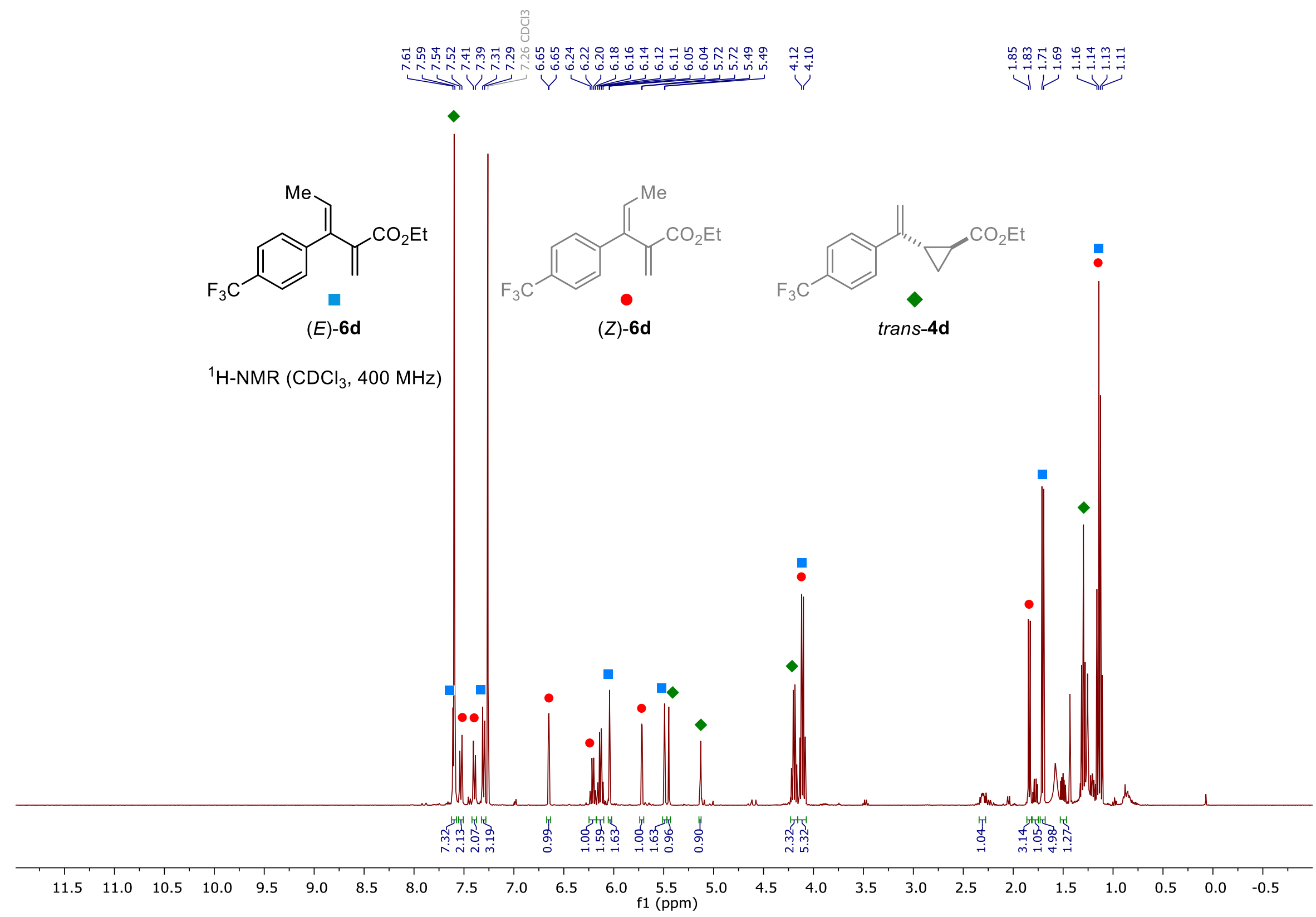




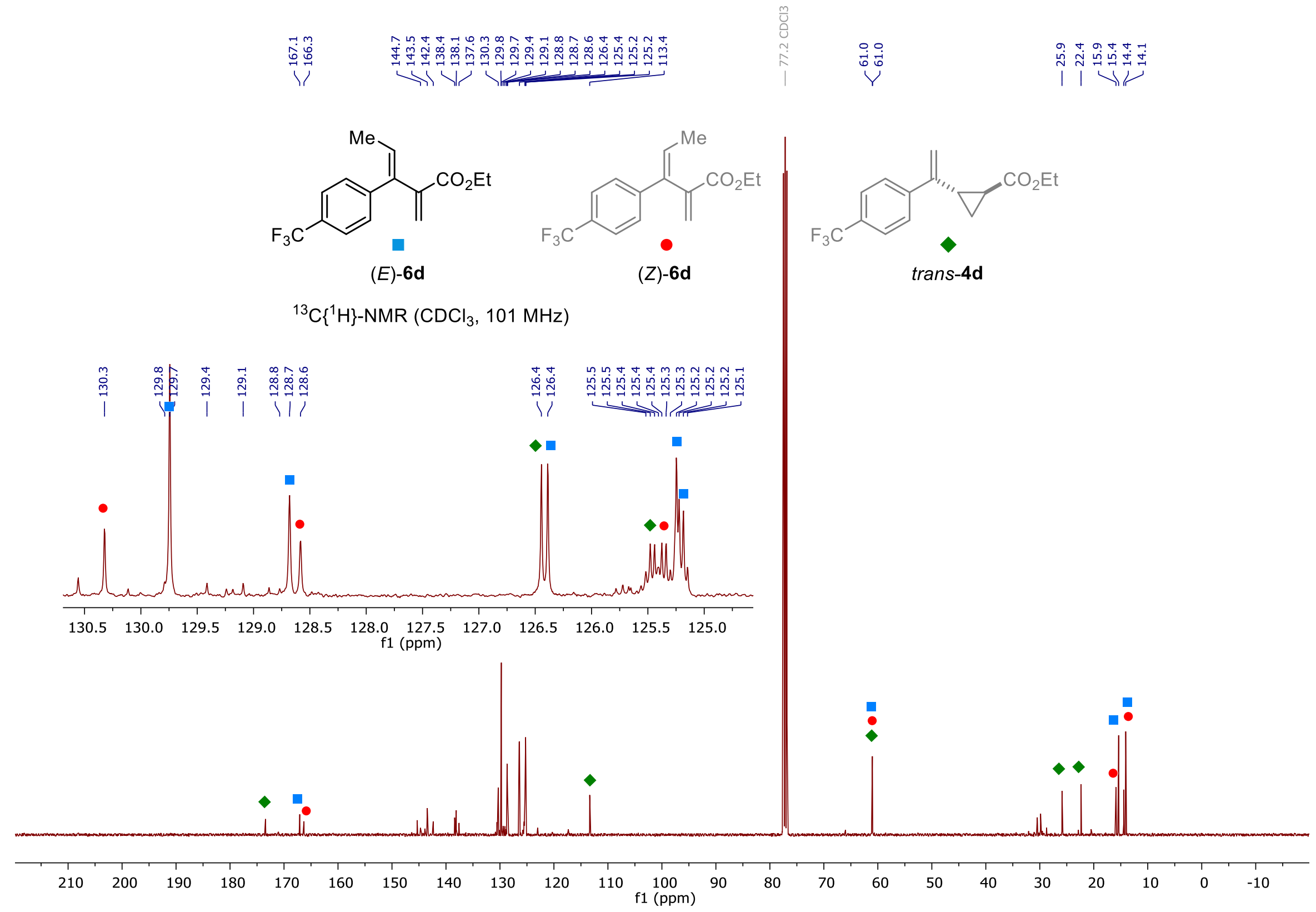



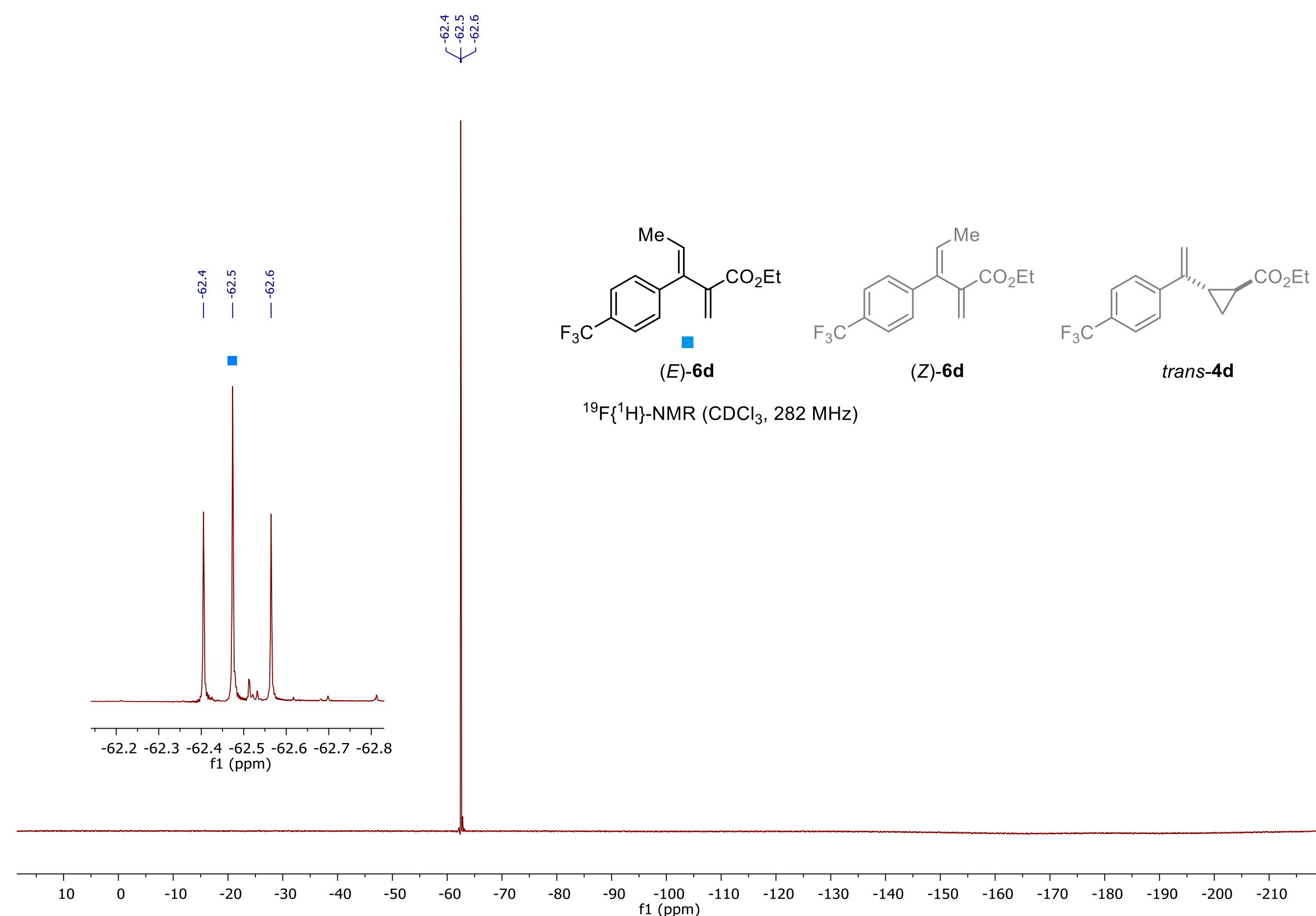


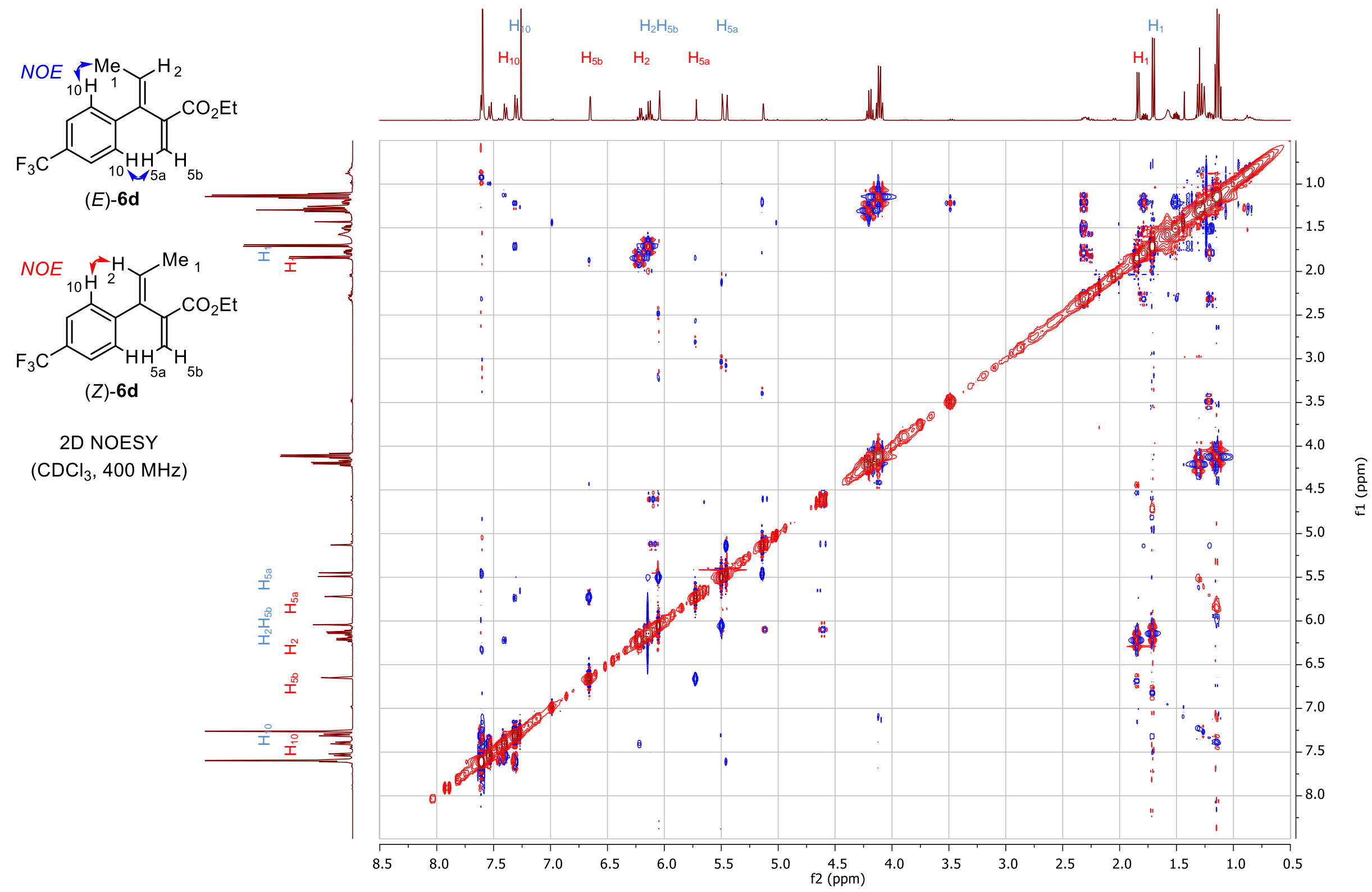




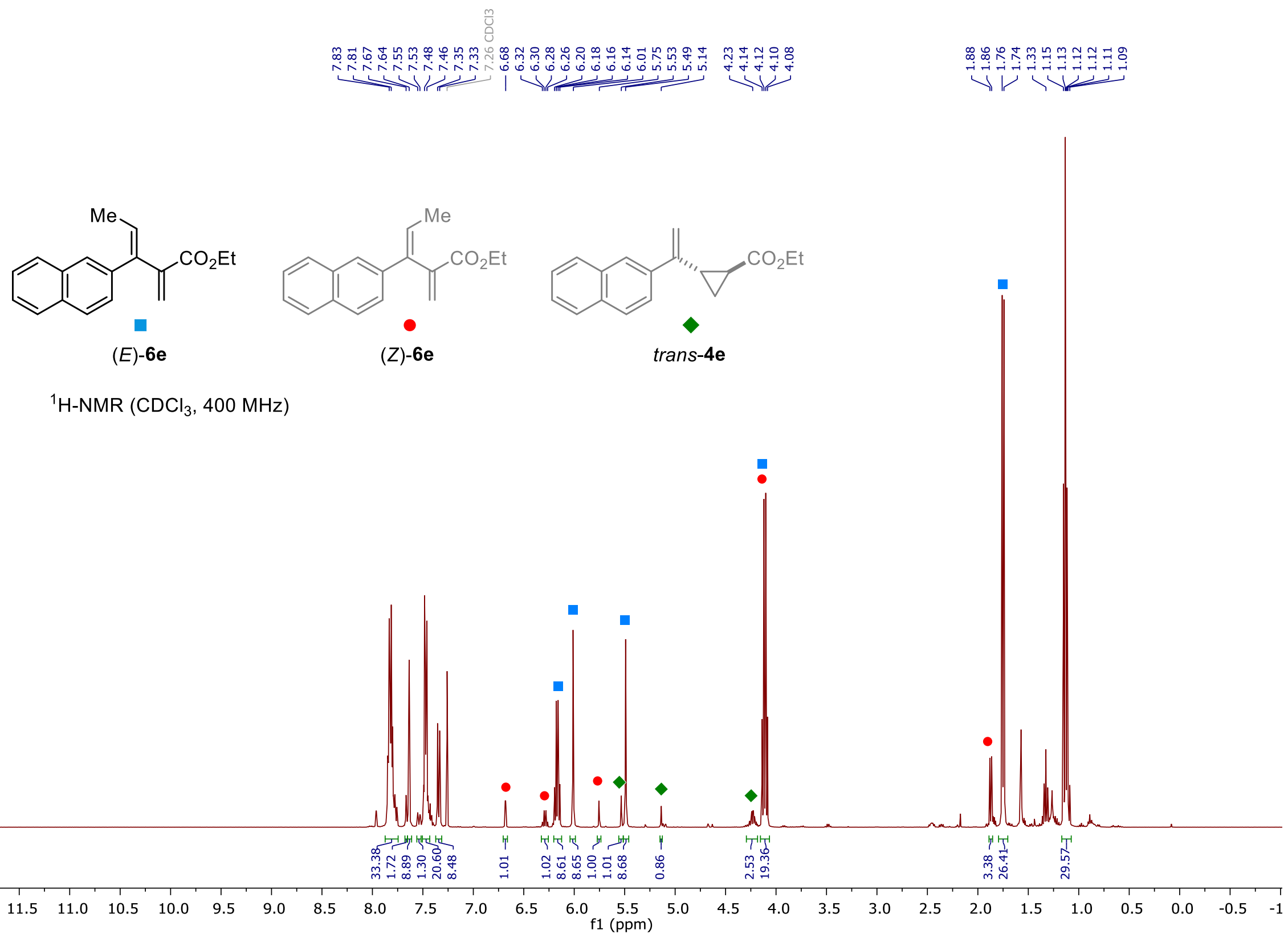




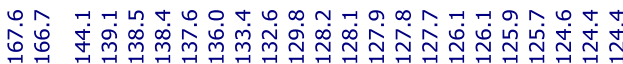

영ำ

䑻织守

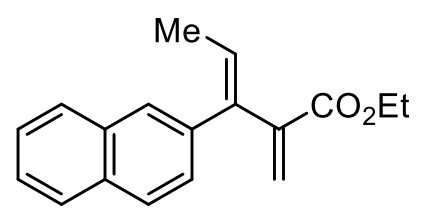

$(E)-6 \mathbf{e}$

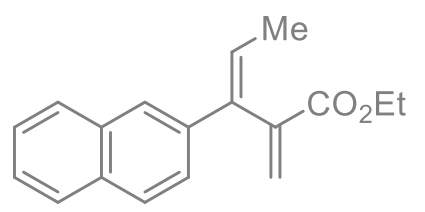

(Z)-6e

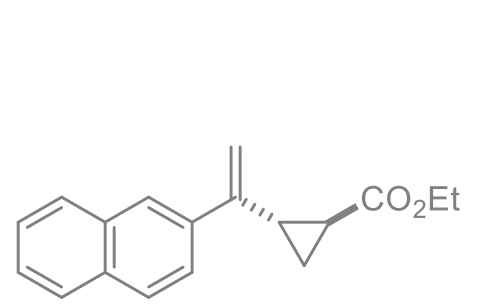

trans-4e

${ }^{13} \mathrm{C}\left\{{ }^{1} \mathrm{H}\right\}-\mathrm{NMR}\left(\mathrm{CDCl}_{3}, 101 \mathrm{MHz}\right)$

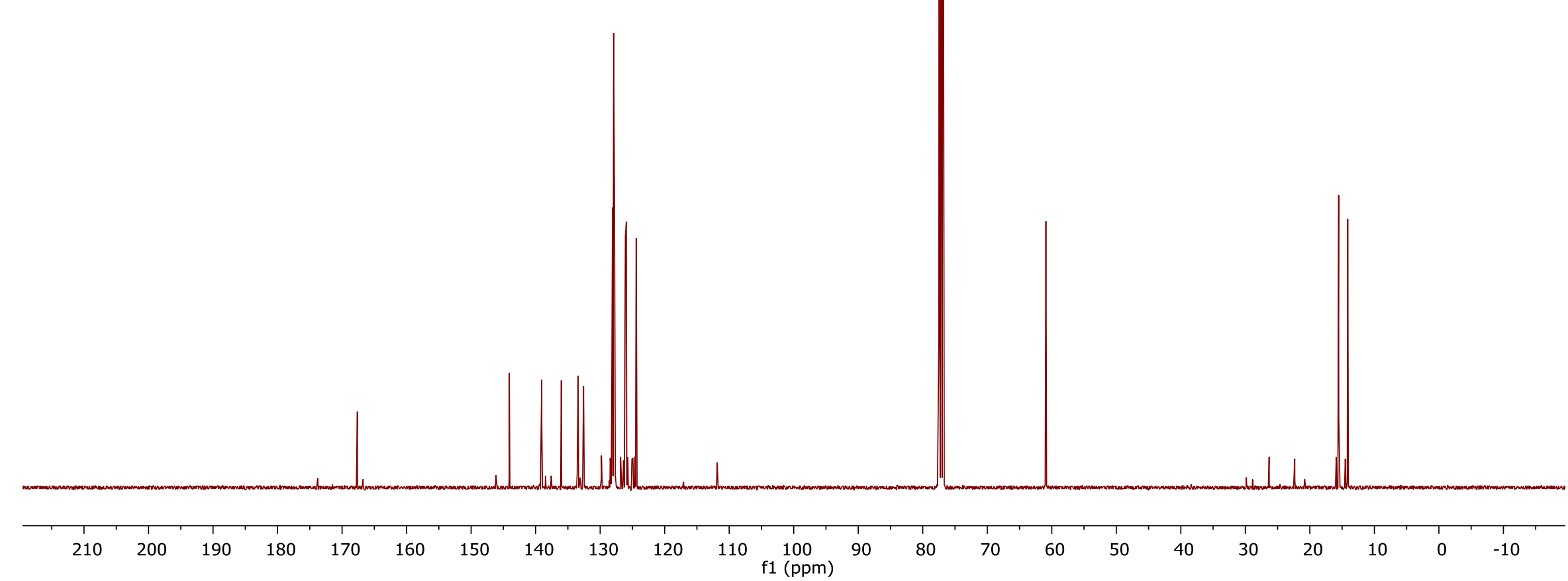




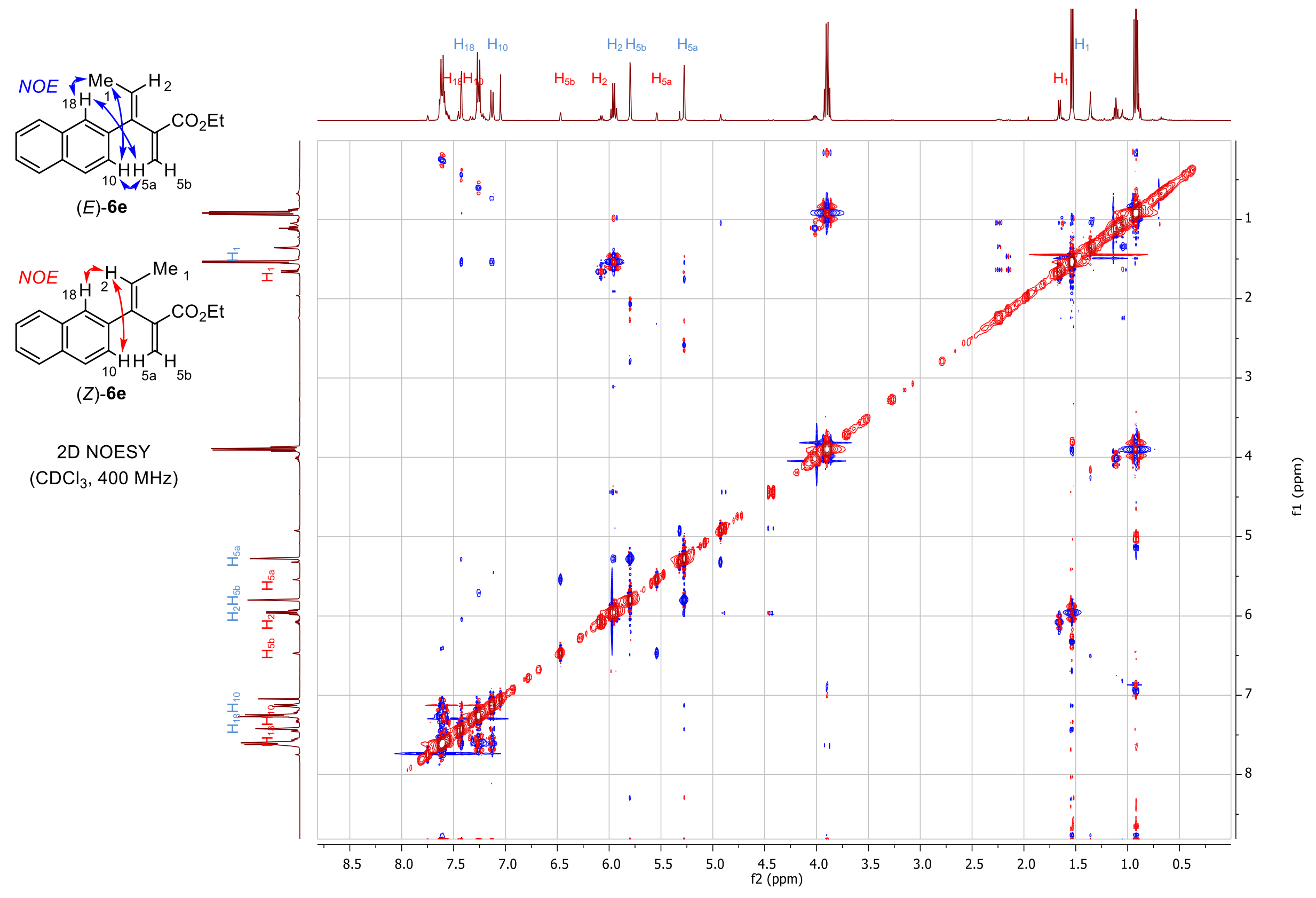




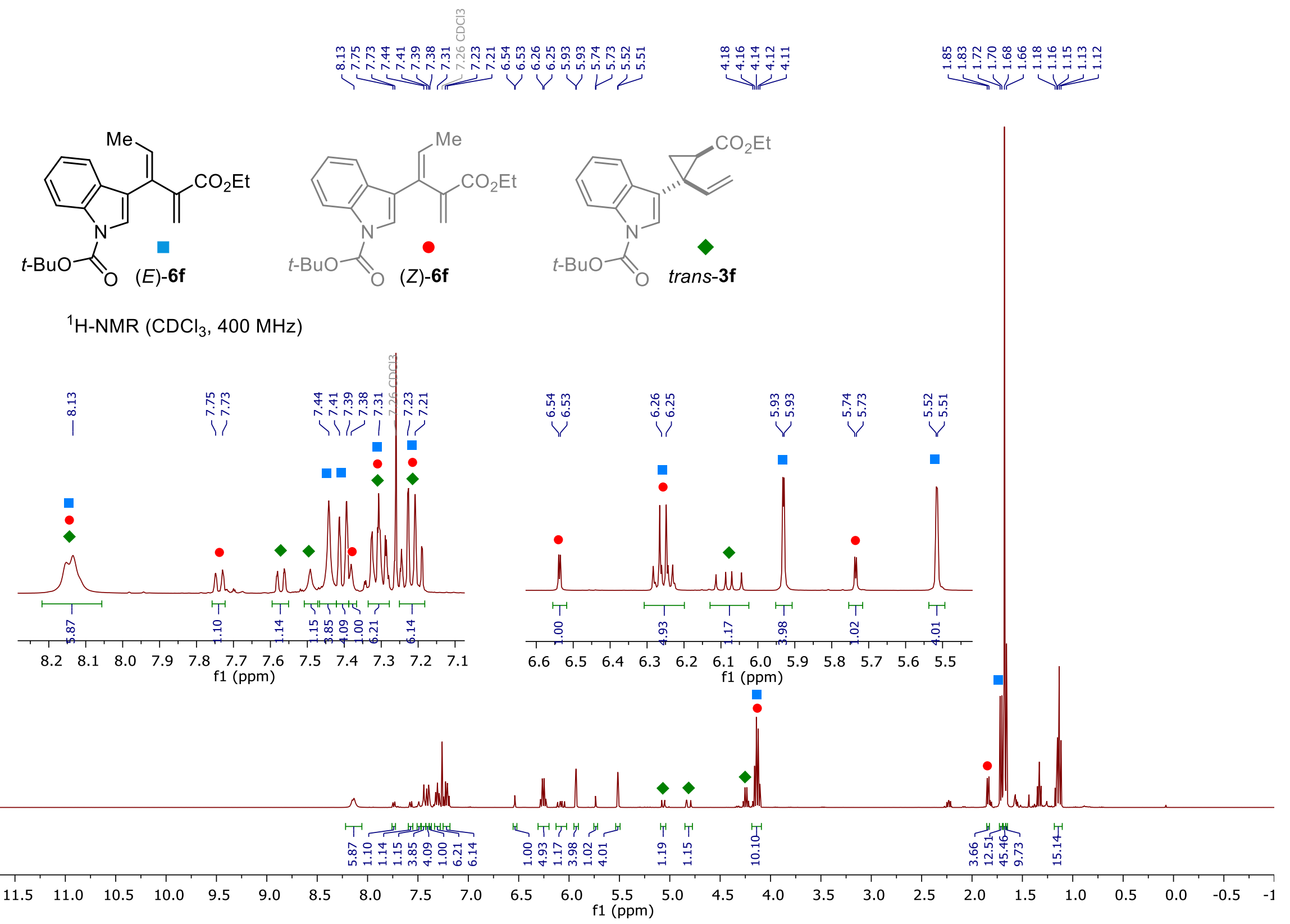




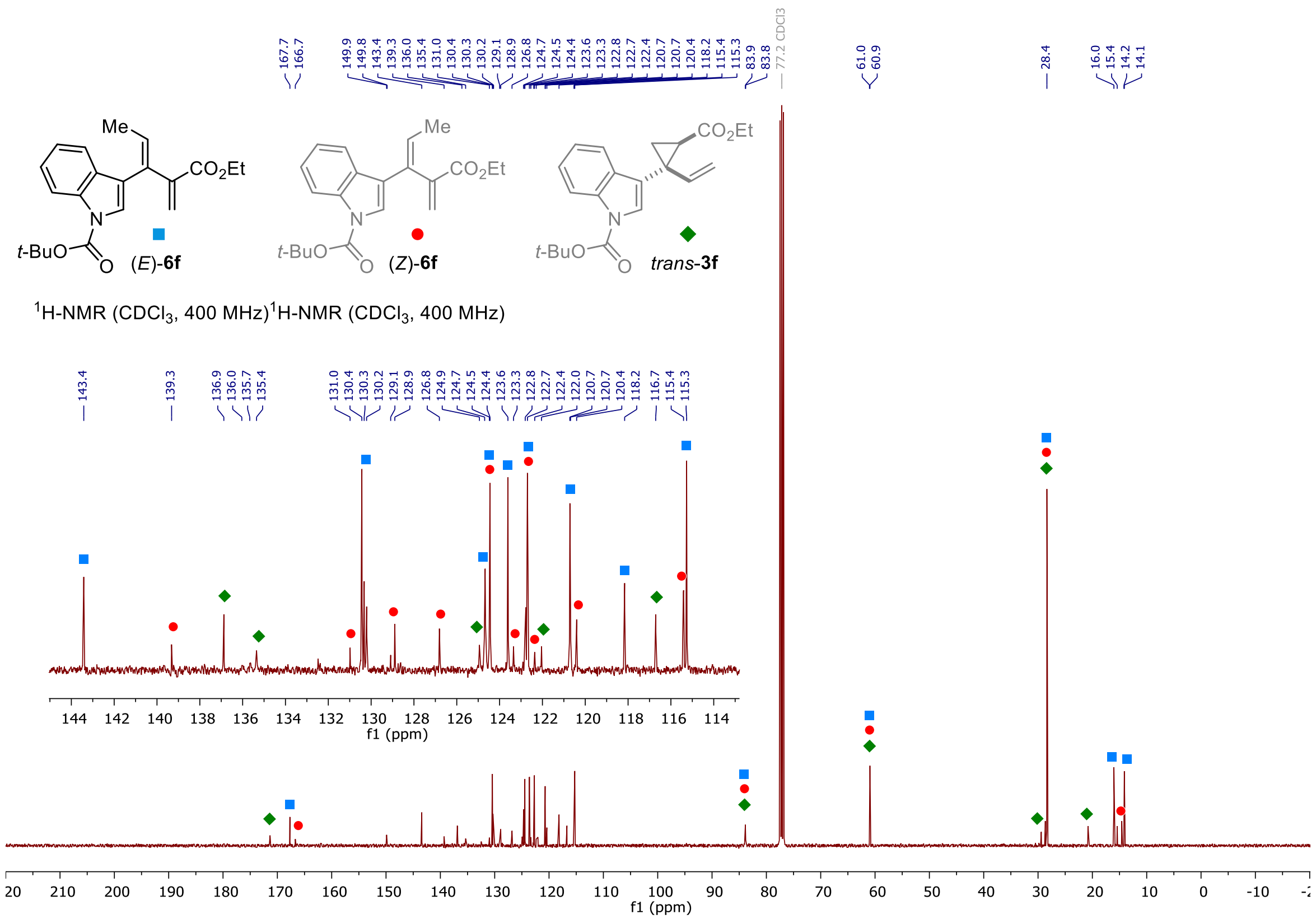




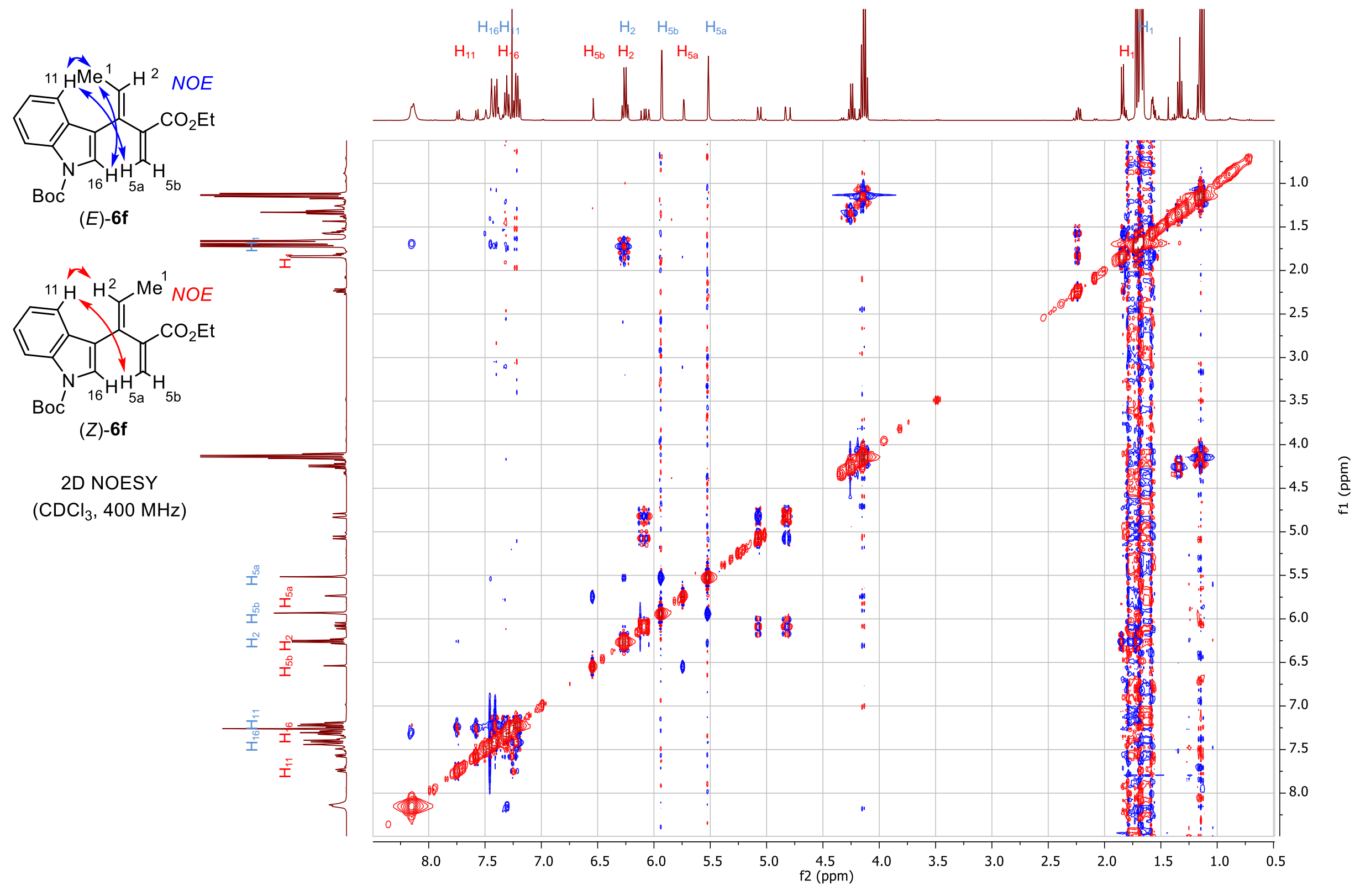


<smiles>C=C(OCC)C(=CC)C(=C)c1ccsc1</smiles>

$(E)-6 \mathbf{g}$

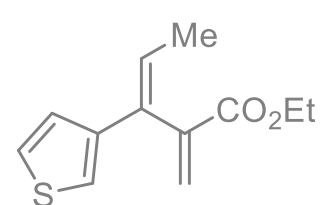

(Z)-6g

${ }^{1} \mathrm{H}-\mathrm{NMR}\left(\mathrm{CDCl}_{3}, 400 \mathrm{MHz}\right)$
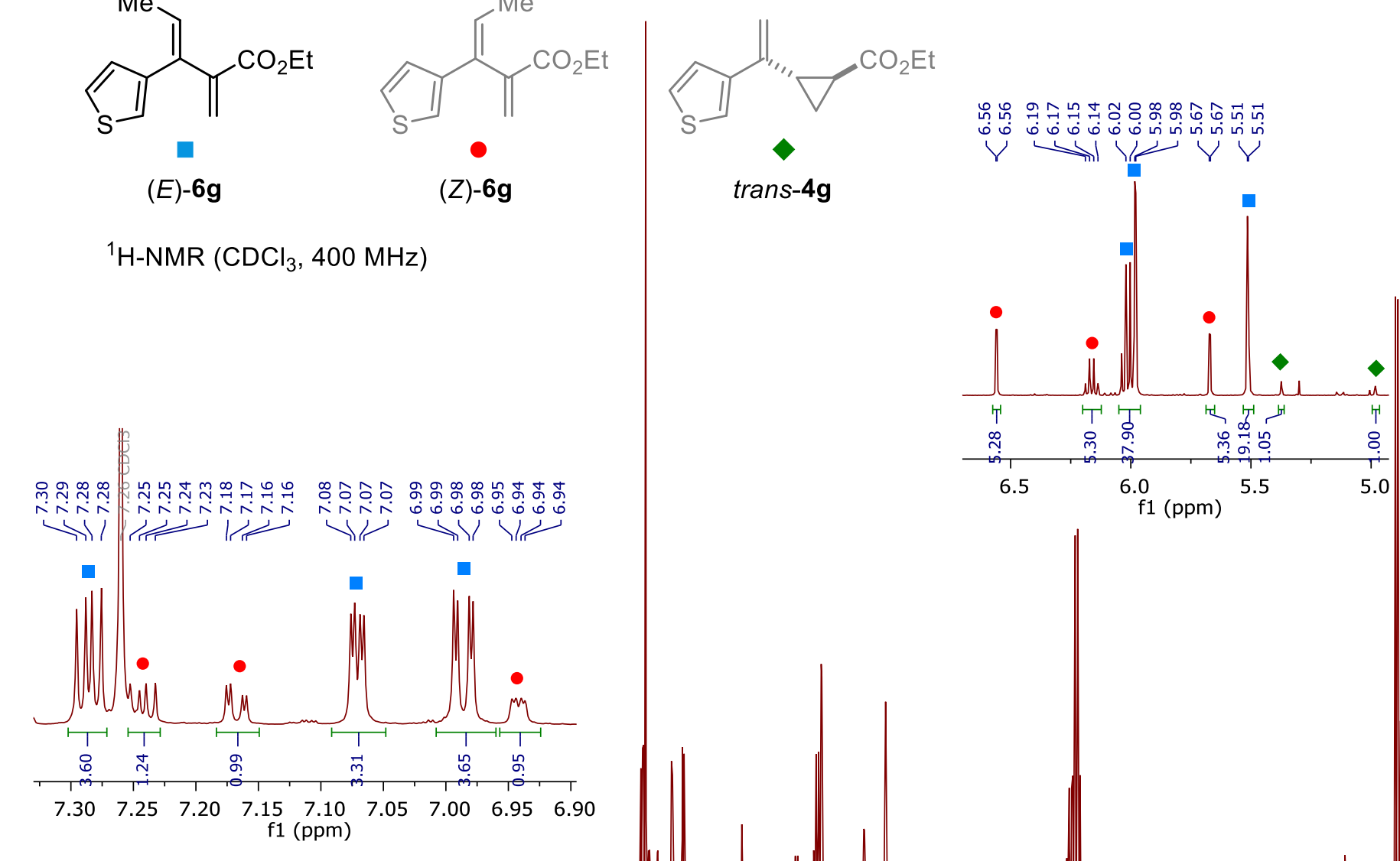

$1(\mathrm{ppm})$

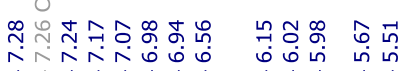

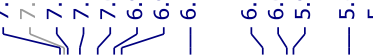

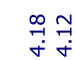

$\underbrace{\infty}$

1

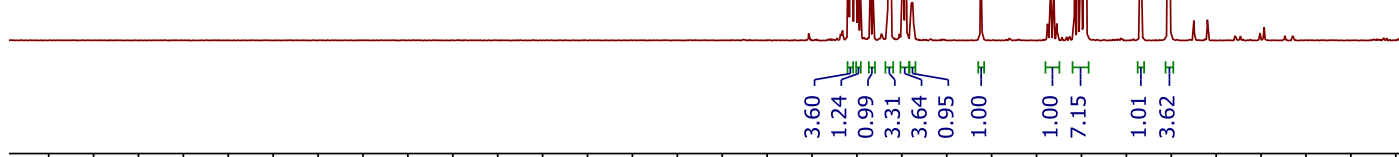

$\begin{array}{lllll}11.5 & 11.0 & 10.5 & 10.0 & 9.5\end{array}$

$6.0 \underset{f 1(\mathrm{ppm})}{5.5}$
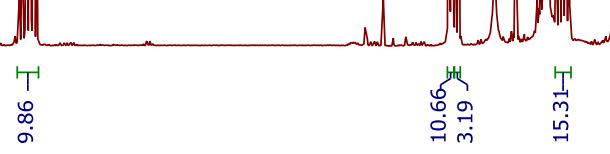

$\begin{array}{llllll}.5 & 1.0 & 0.5 & 0.0 & -0.5 & -1\end{array}$ 


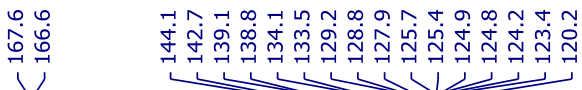

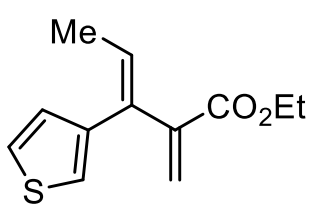

(E)-6g

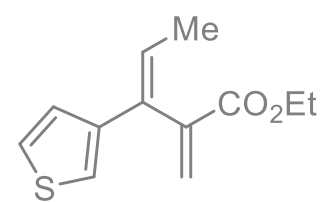

(Z)-6g

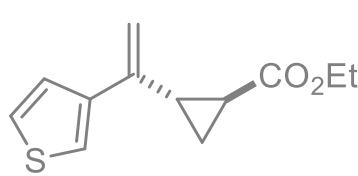

trans-4g

${ }^{13} \mathrm{C}\left\{{ }^{1} \mathrm{H}\right\}-N M R\left(\mathrm{CDCl}_{3}, 101 \mathrm{MHz}\right)$

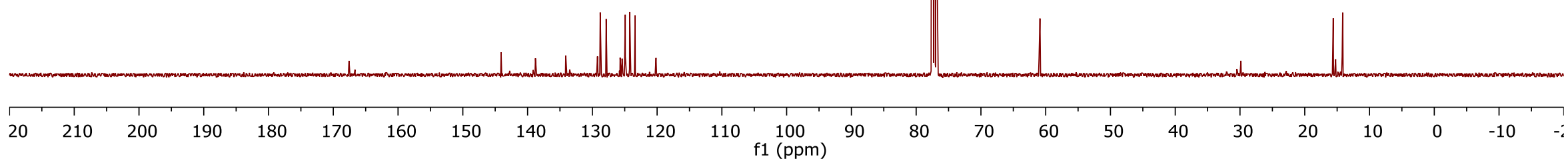




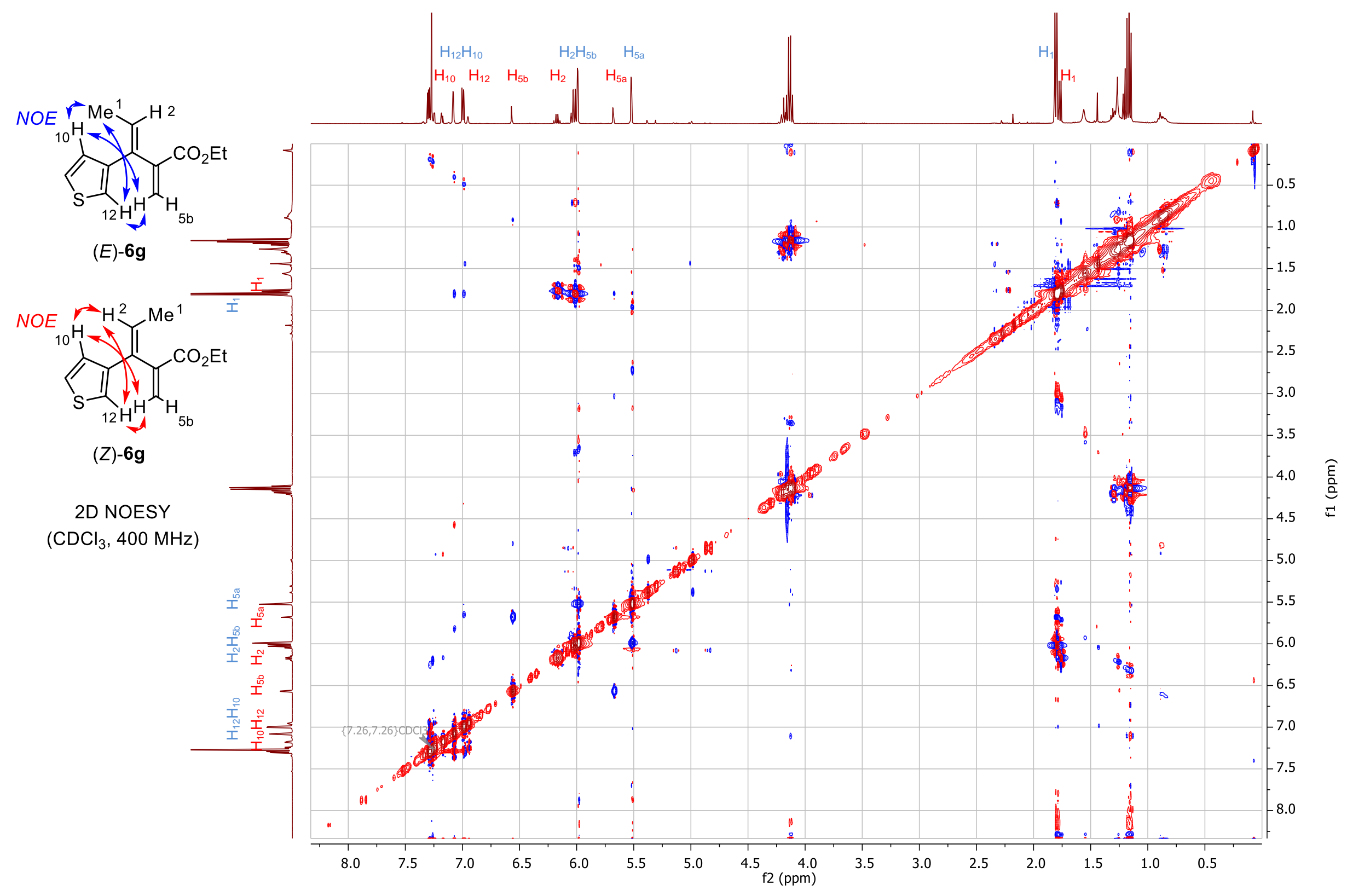




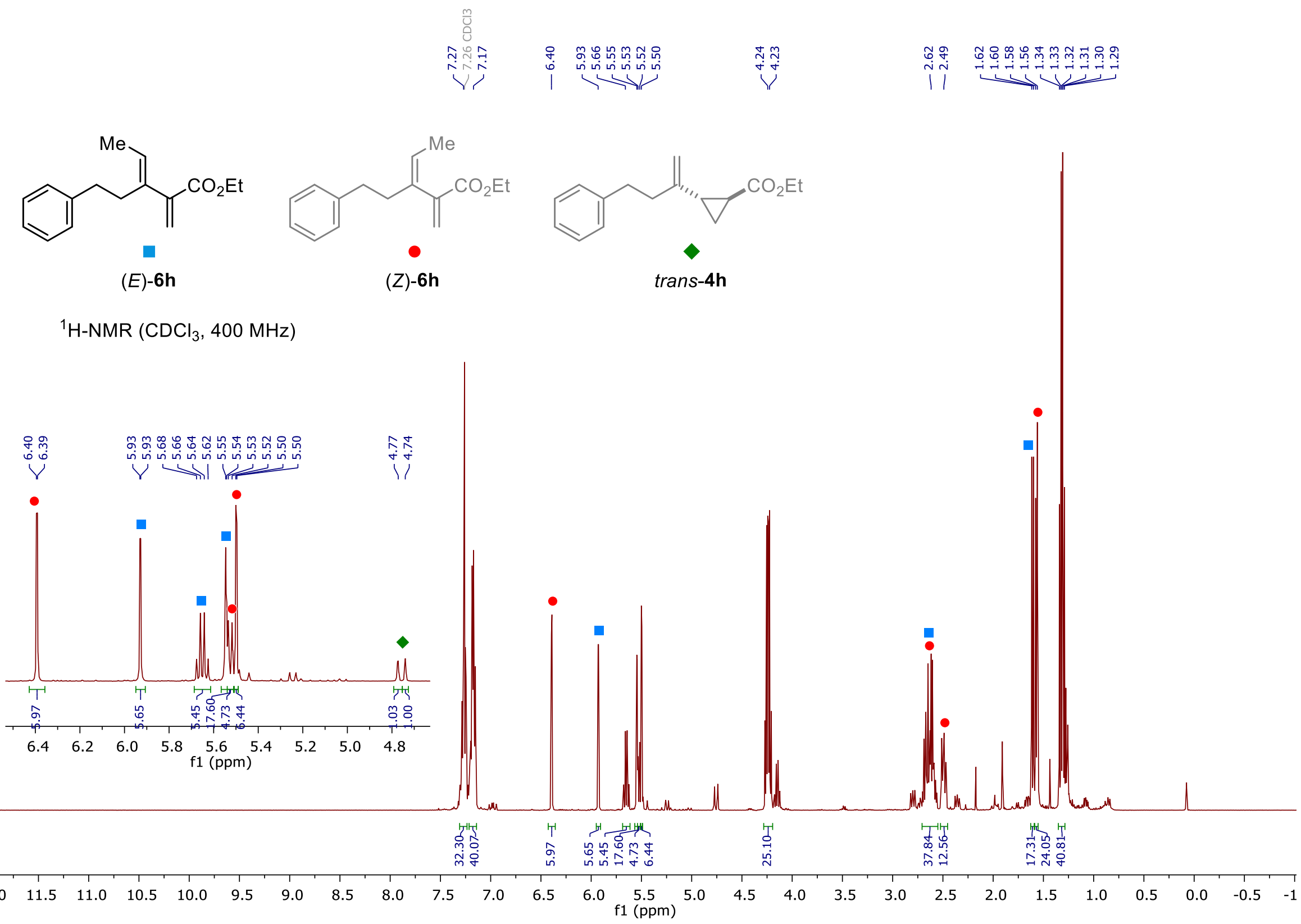




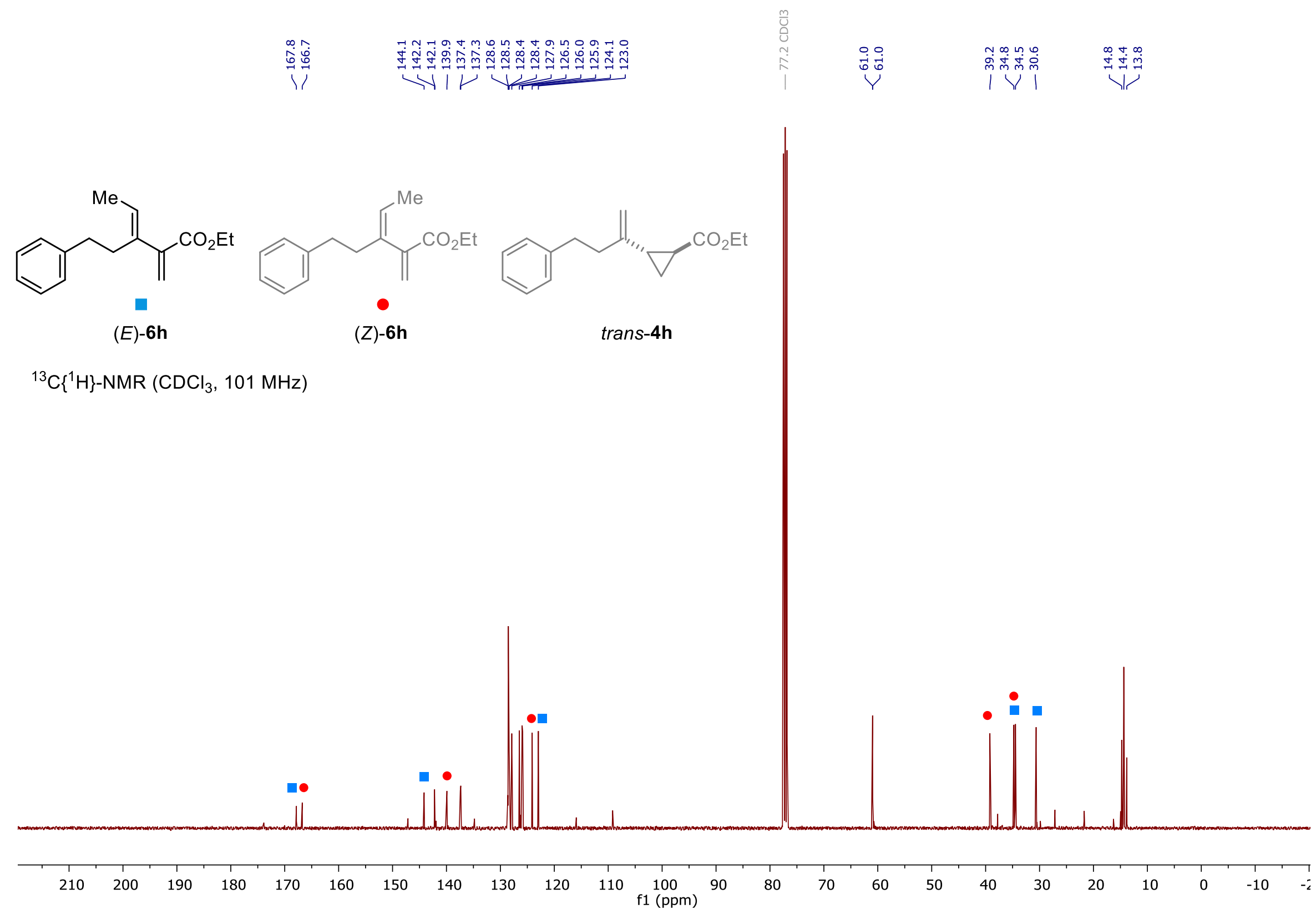


NOE

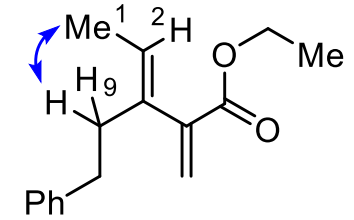

(E)-6h
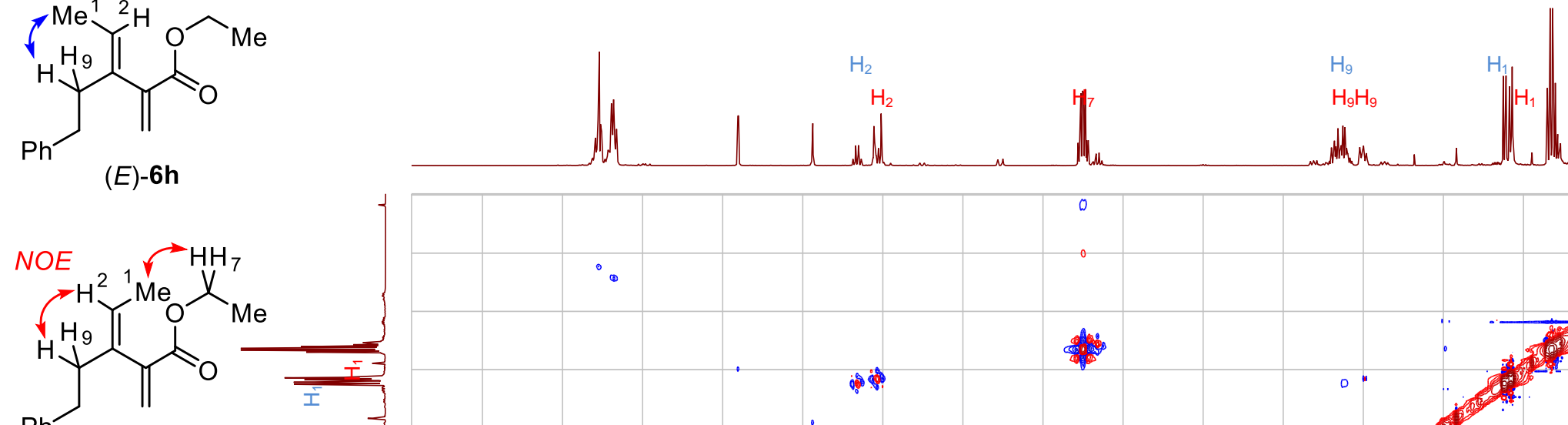

(Z)-6h

2D NOESY $\left(\mathrm{CDCl}_{3}, 400 \mathrm{MHz}\right)$

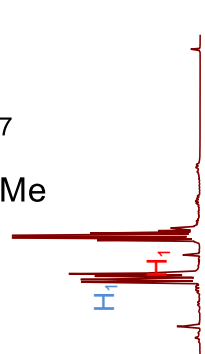

$$
\text { 政 }
$$

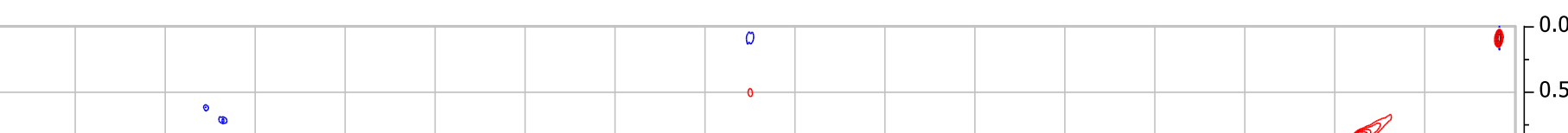

오 옹

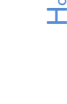

.

$-$

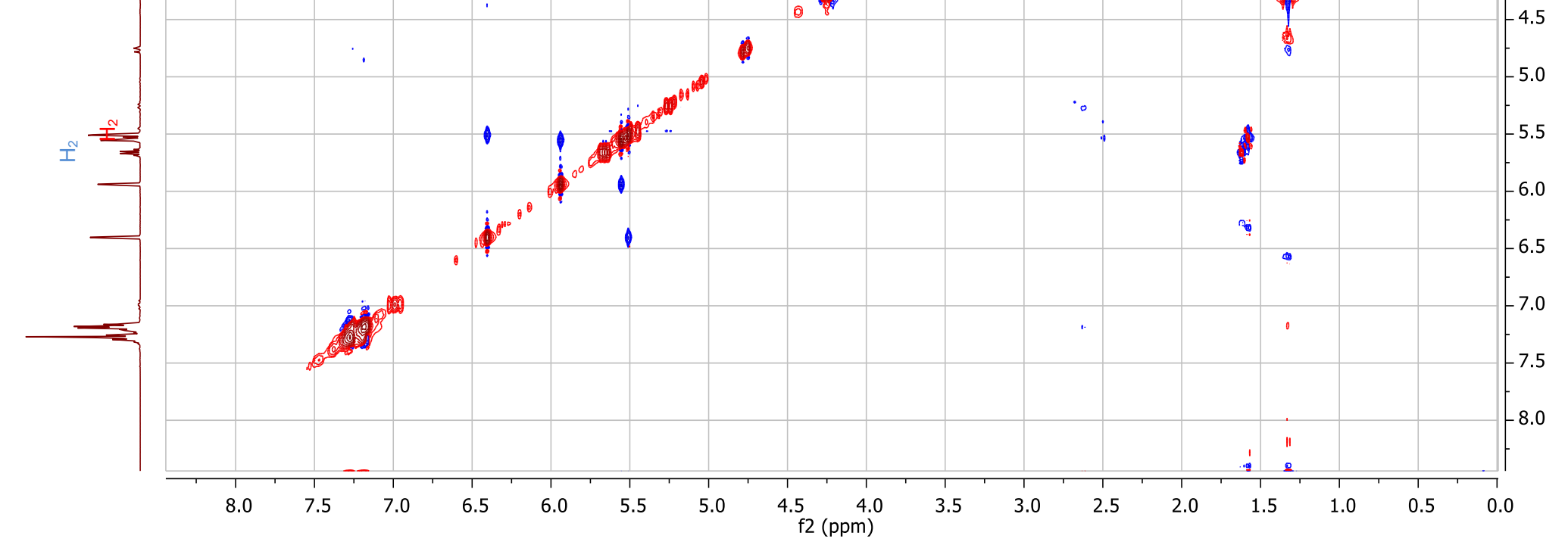




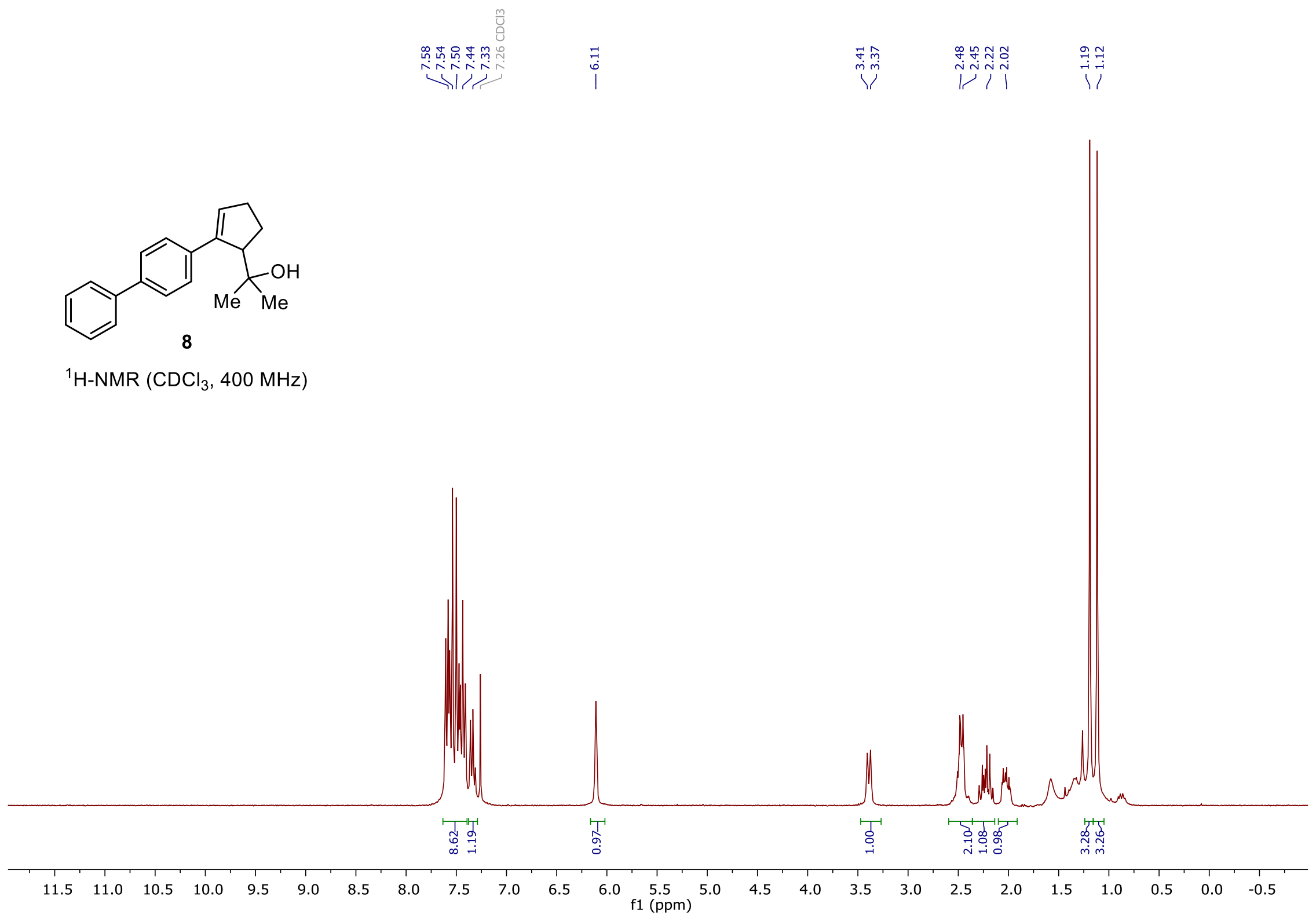



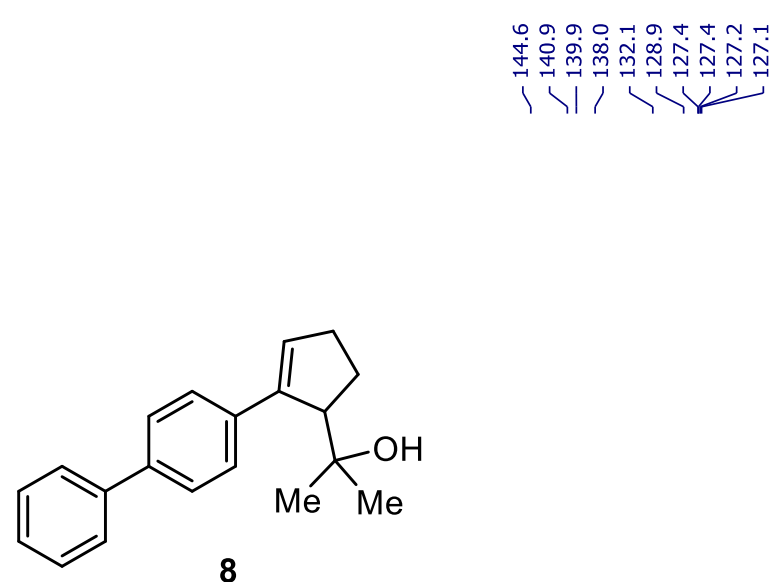

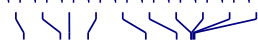

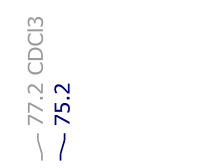

i

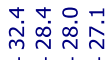

${ }^{13} \mathrm{C}\left\{{ }^{1} \mathrm{H}\right\}-\operatorname{NMR}\left(\mathrm{CDCl}_{3}, 101 \mathrm{MHz}\right)$

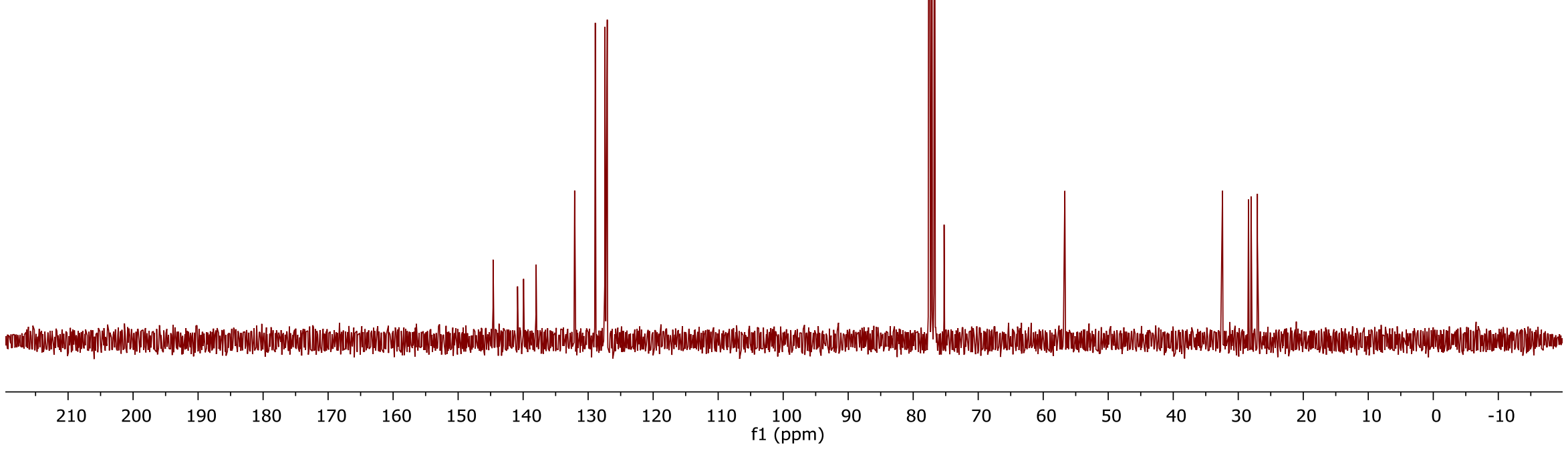


Supporting Information

$\mathrm{S} 80$
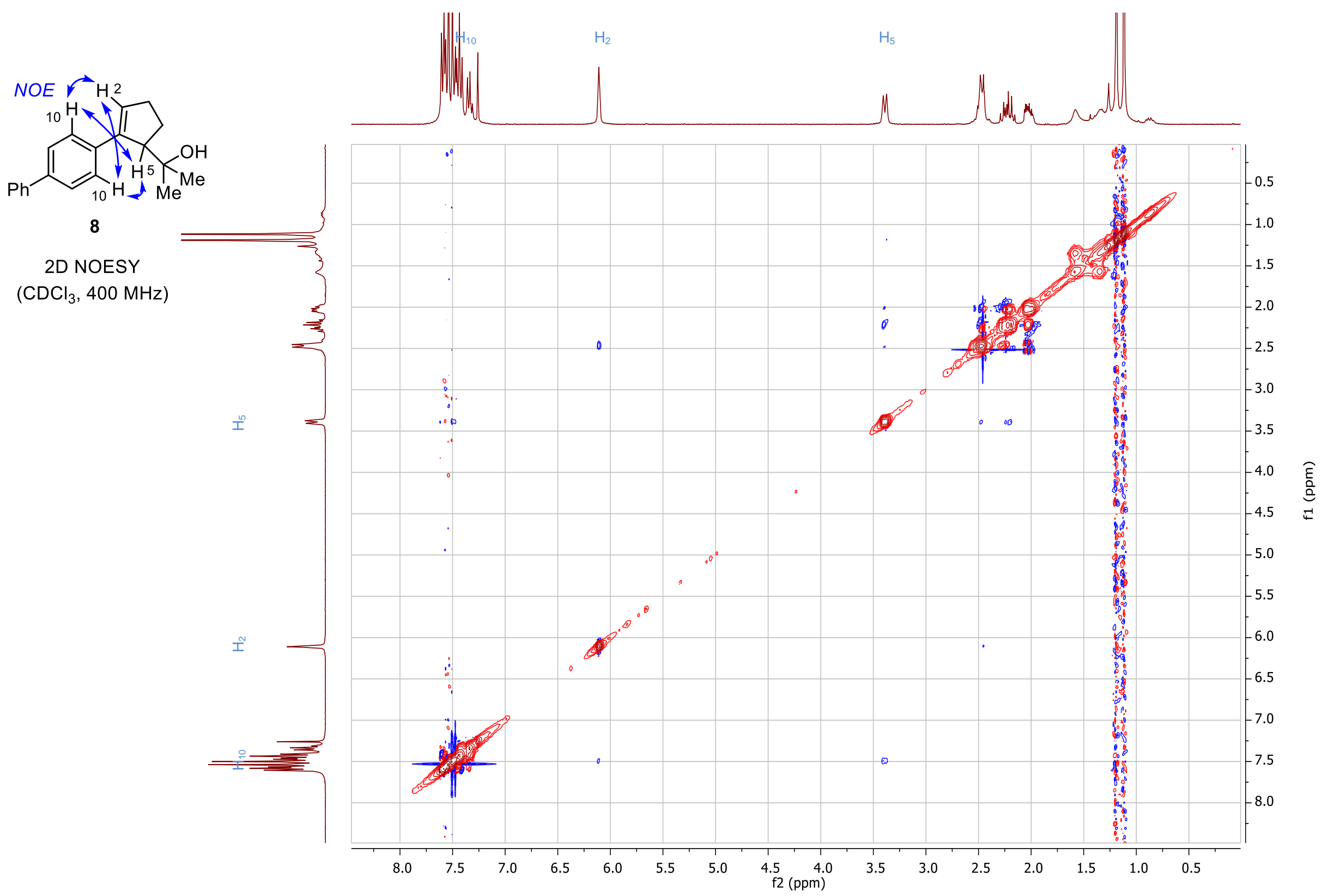


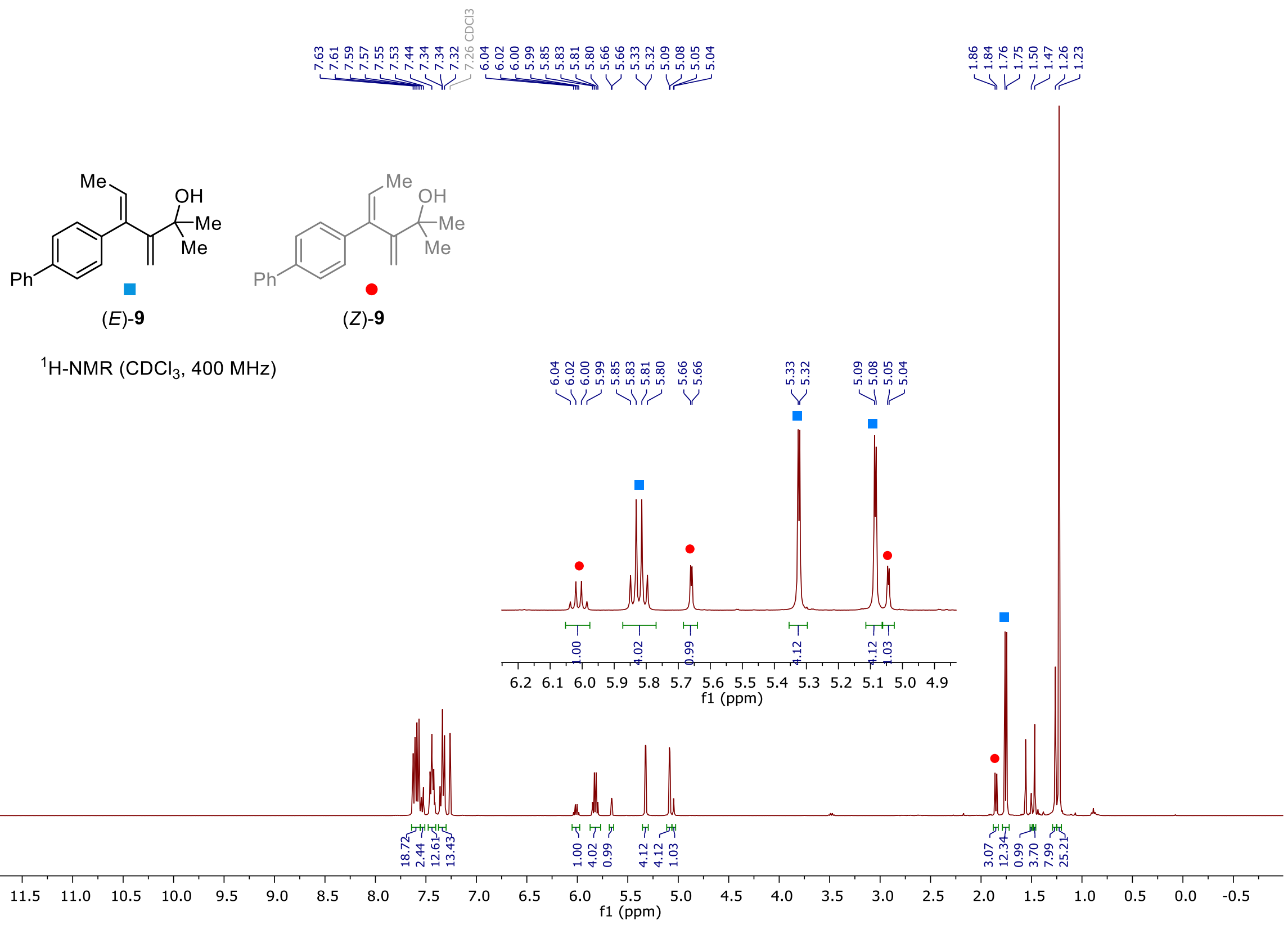




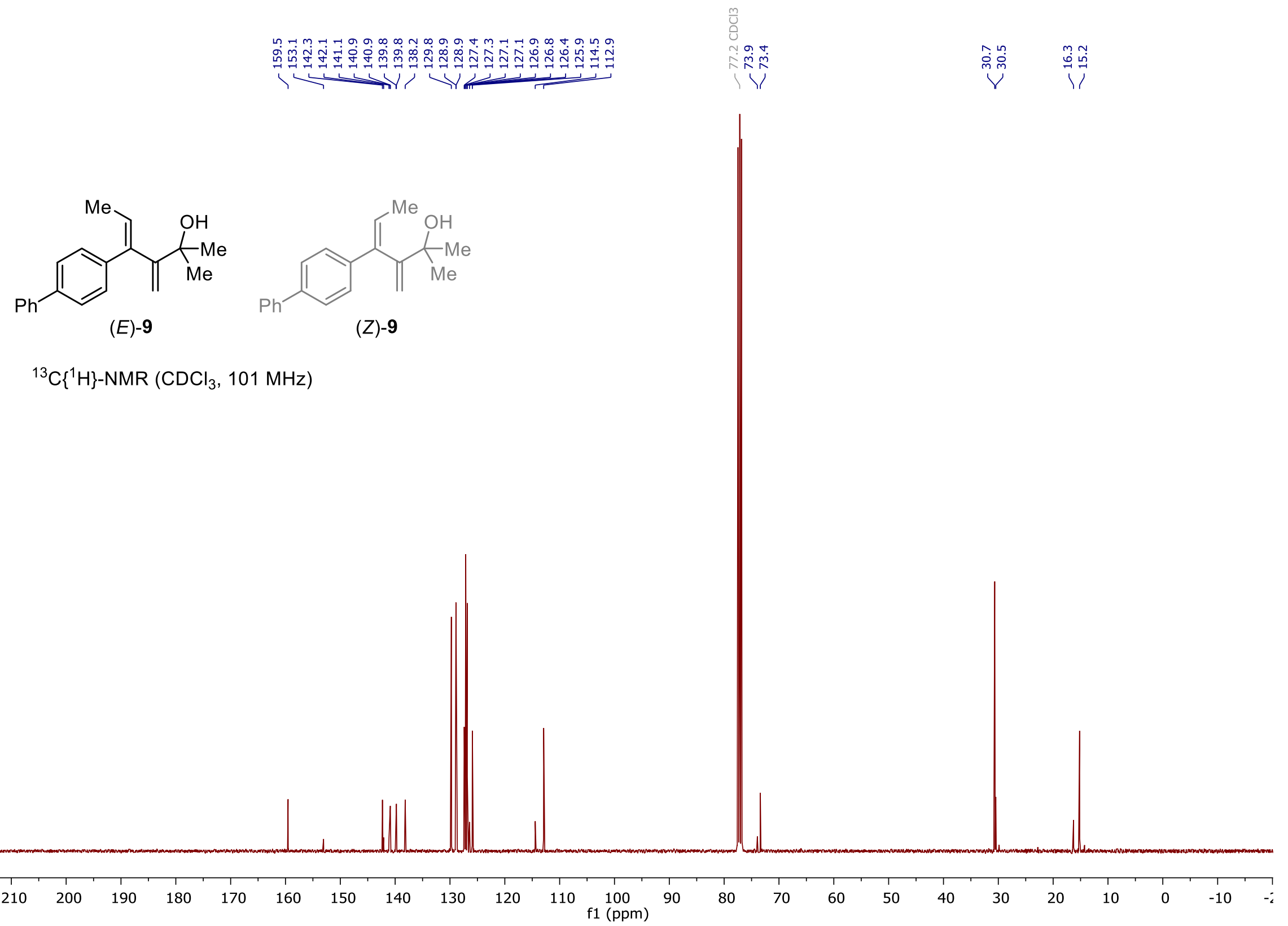




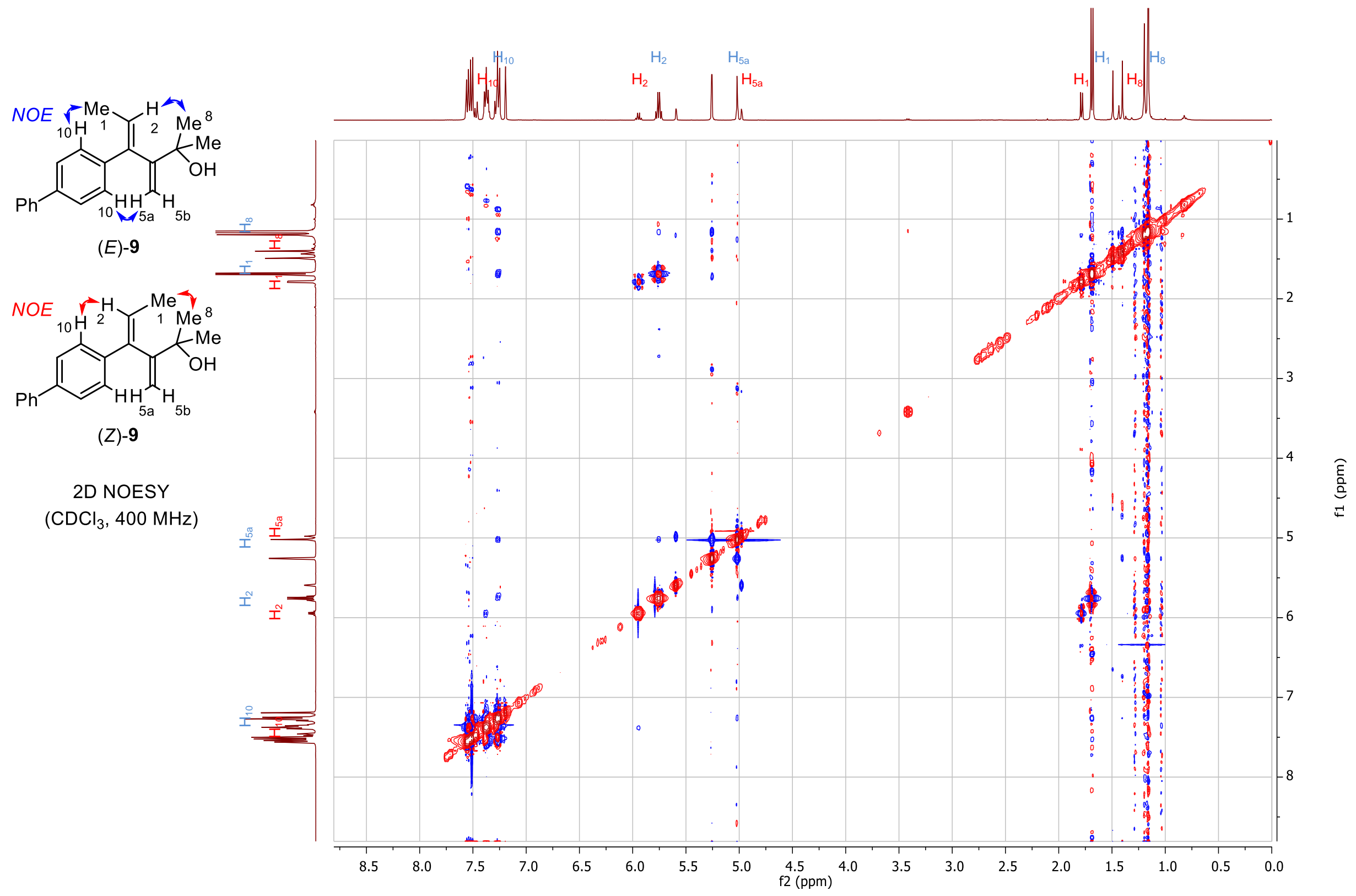




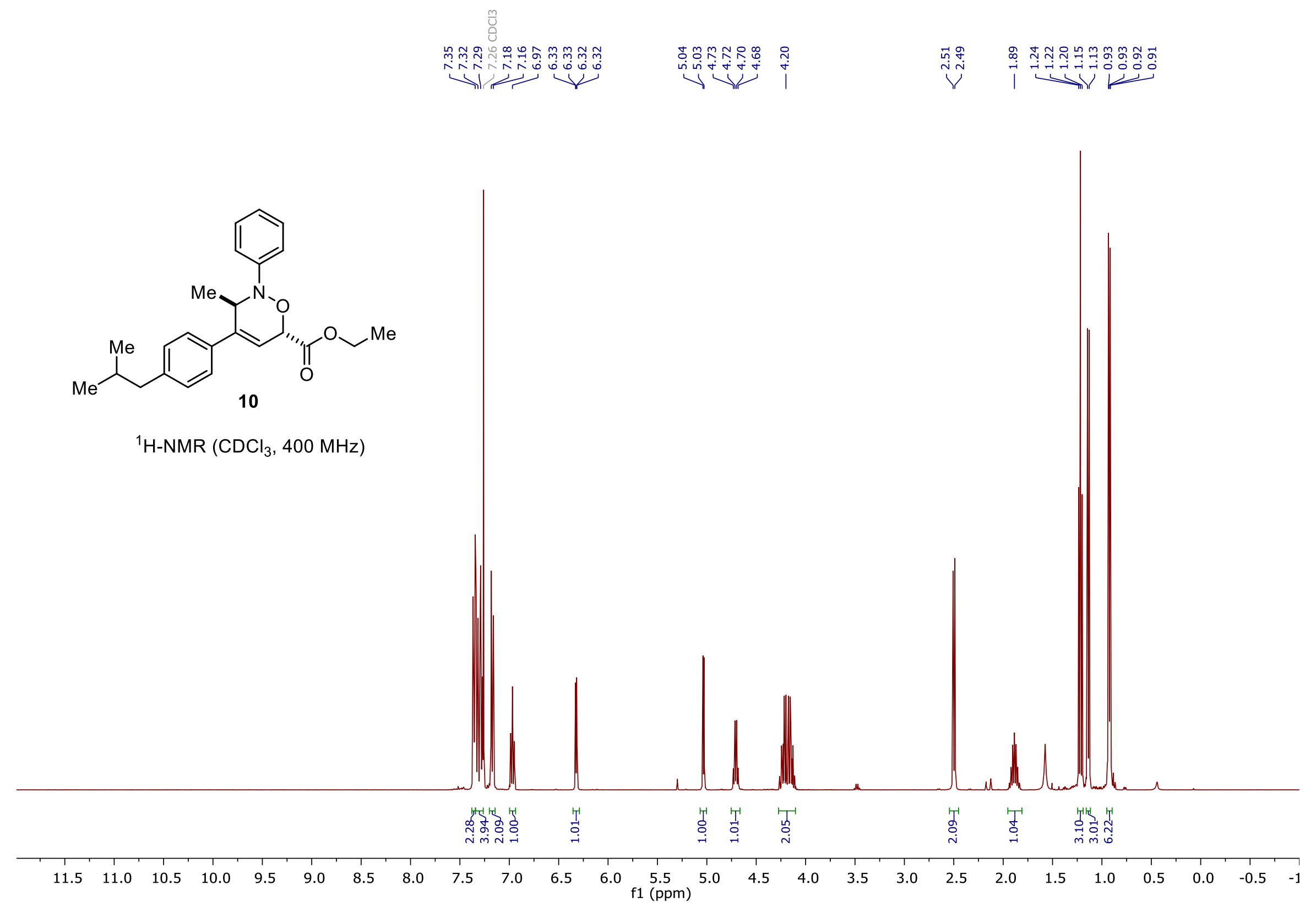




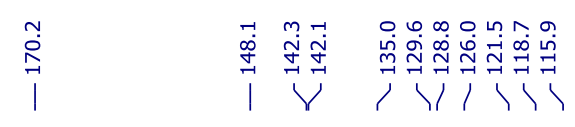

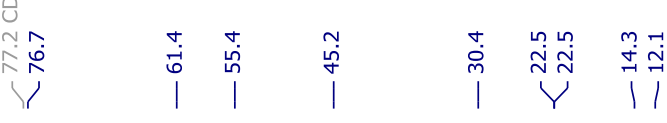

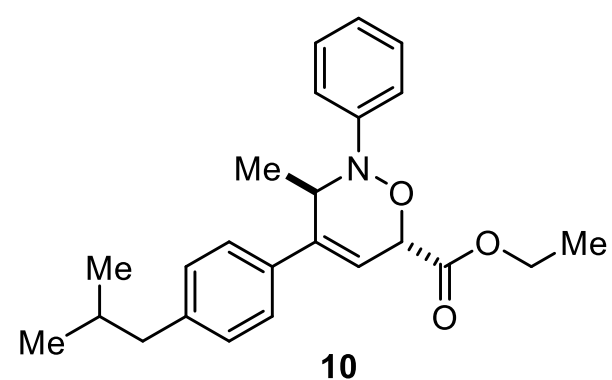

${ }^{13} \mathrm{C}\left\{{ }^{1} \mathrm{H}\right\}-\mathrm{NMR}\left(\mathrm{CDCl}_{3}, 101 \mathrm{MHz}\right)$

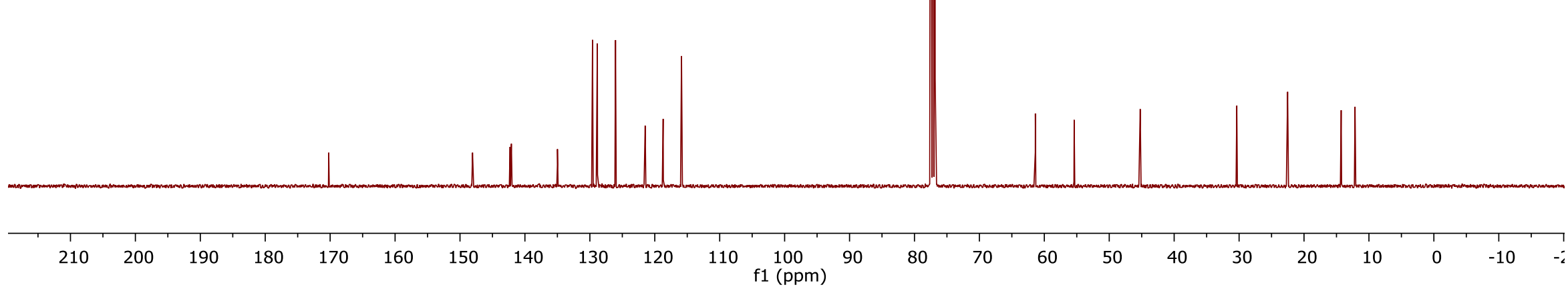




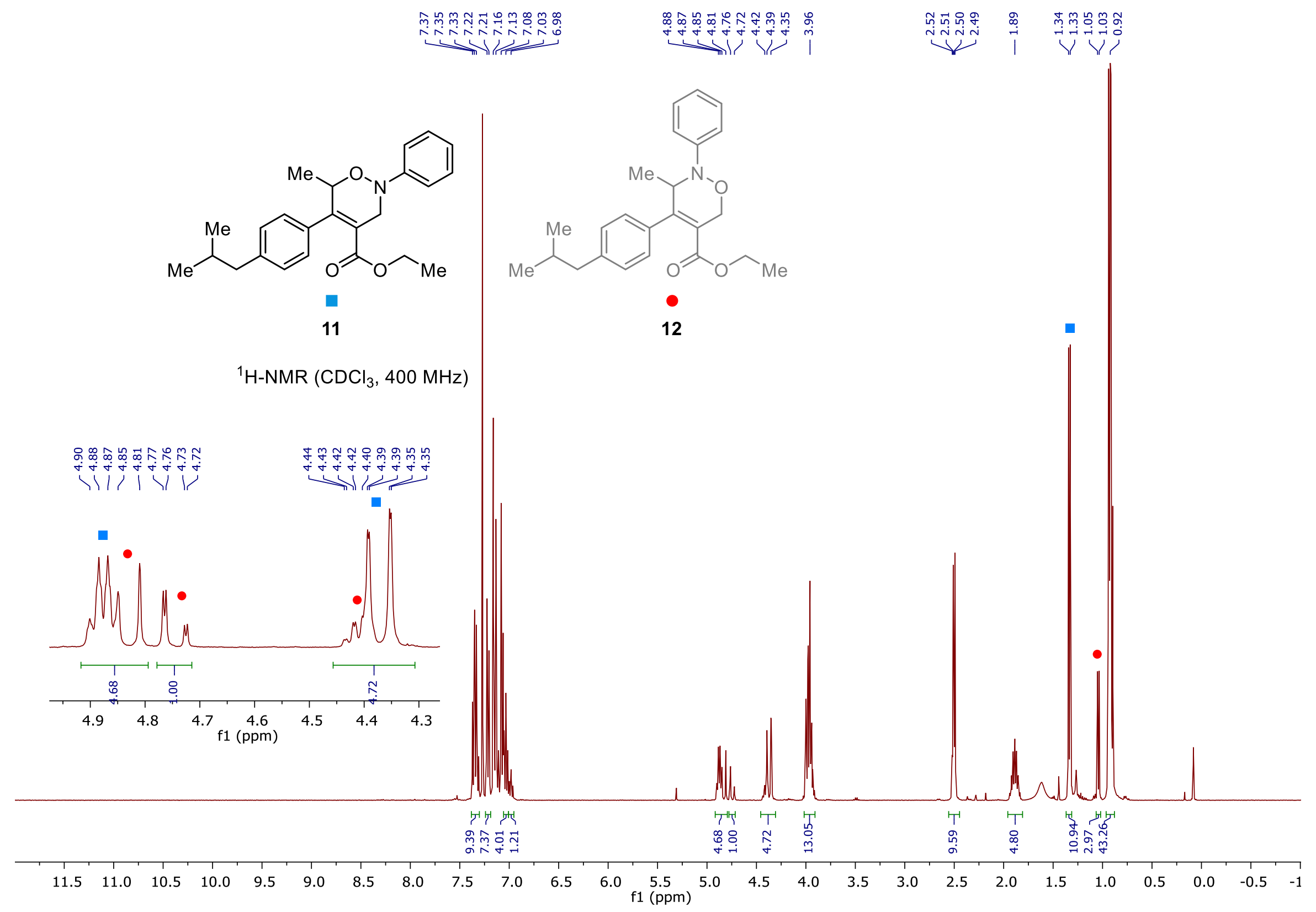




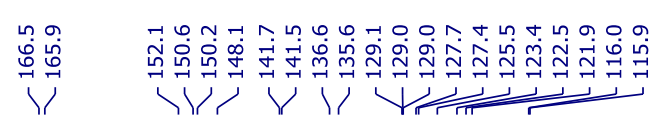

ill
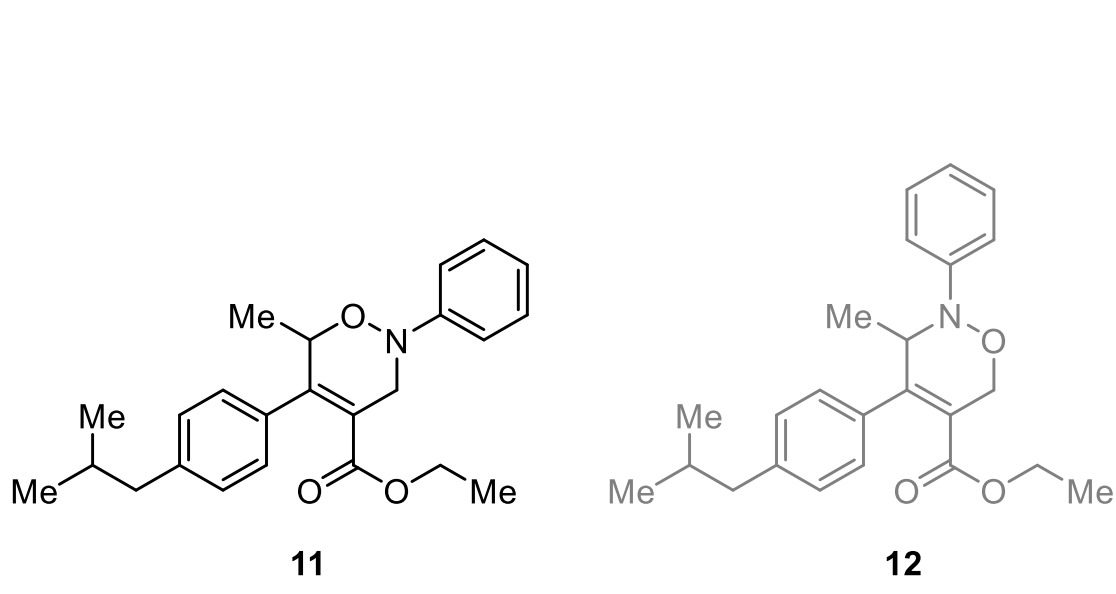

${ }^{13} \mathrm{C}\left\{{ }^{1} \mathrm{H}\right\}-N M R\left(\mathrm{CDCl}_{3}, 101 \mathrm{MHz}\right)$

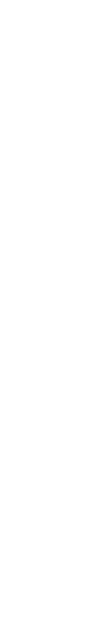

)
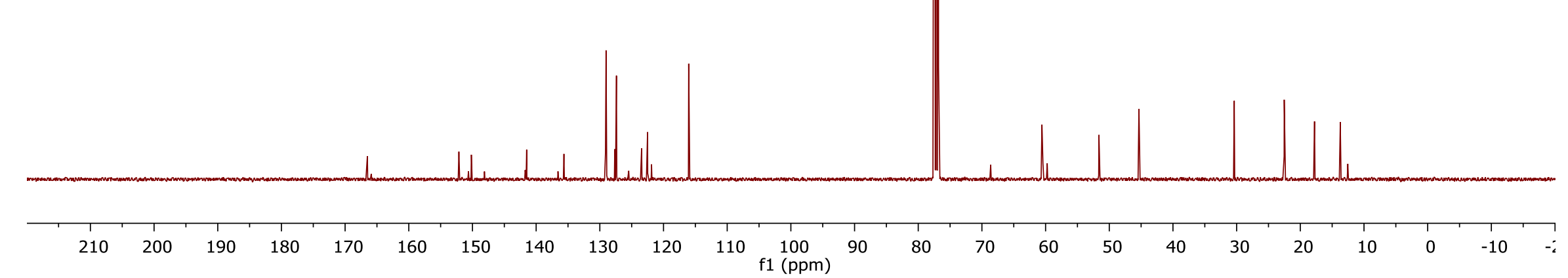